\title{
Fabrication and Characterization of Nanostructured Silicon Films For Photovoltaic Applications
}

\author{
by \\ Kai Wang
}

A thesis submitted to the Faculty of Graduated and Postdoctoral Affairs in partial fulfillment of the requirements for the degree of

Master of Applied Science

In

Electrical and Computer Engineering

Carleton University

Ottawa, Ontario

(C)2014 


\section{Abstract}

In semiconductor solar cells, the energy band structure has a major influence on cell efficiency. Shifting the bandgap of the semiconductor to fit the solar radiation spectral will increase the energy conversion efficiency. One way to modify the bandgap is by nanostructuring the material. In this thesis, we introduce a novel nanostructured ITO substrate deposited with an ultrathin silicon film. The glancing angle deposition research group fabricated the ITO substrate at University of Alberta while the amorphous Si film was deposited at Carleton University. The sample was fabricated for optical property study and photovoltaic performance evaluation.

An optical absorption and reflection characterization system was set up to measure the thin film semiconductor. The system was based on programmable optical equipment. The fabricated nanostructured Si/ITO film was characterized with this set up and the optical constants such as absorption coefficient and refractive index were extracted.

Although the structure of the deposited Si film was not exactly as expected, the measurements have shown slight enhancement of visible band light absorption. Both a-Si and c-Si were deposited; the absorption of a-Si is $10 \%$ more than c-Si in the visible range. Therefore, the novel a-Si film sample is a promising candidate for further research. 


\section{Acknowledgements}

First of all, I would like to thank my supervisors Dr. Garry Tarr and Dr. Steve McGarry for their advice and help on my thesis over the last two years. They have been always nice and patient when I need their help and advice, I learned a lot from their way of looking at the problem.

I would also like to give thanks to Dr. Michael Brett and his GLAD Research Group. Our sample film could not have been fabricated without their help.

Special thanks go to Rob Vandusen and Rick Adam from Carleton University Micro Fabrication Facility. They helped me a lot in fabricating the nanostructured silicon film.

Thanks also go to Eduardo Barrera. He is kind and helpful, and always around when I need help. I learned a lot from him on how to operate optical equipment in the Laser Optical Lab and how to program VEE.

Kai Wang

Carleton University

November 2013 


\section{Table of Contents}

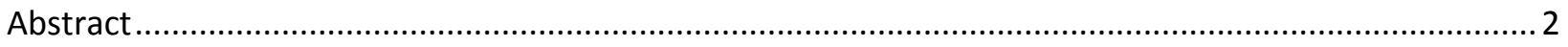

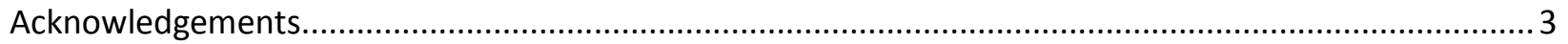

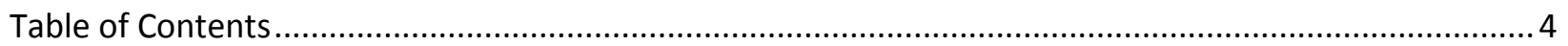

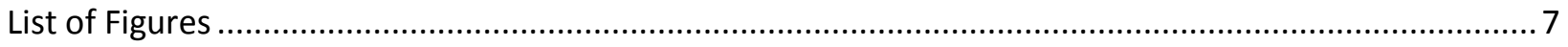

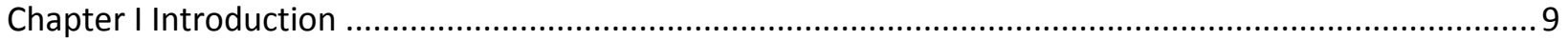

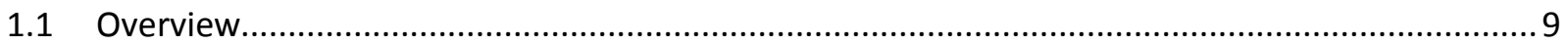

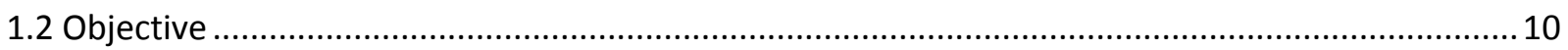

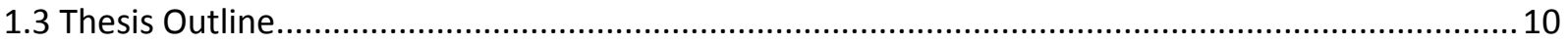

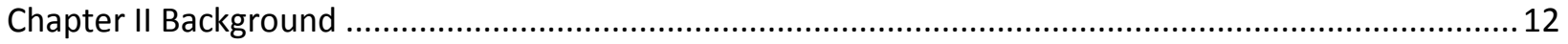

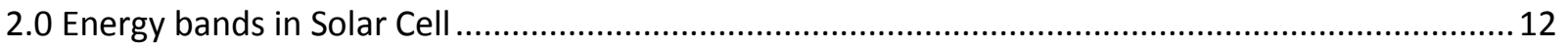

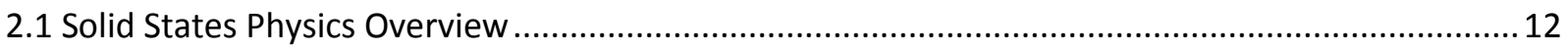

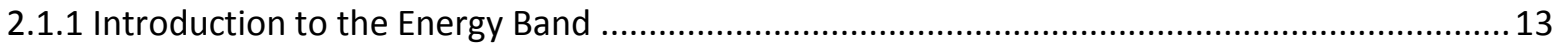

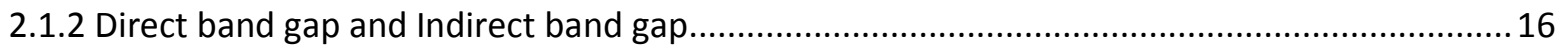

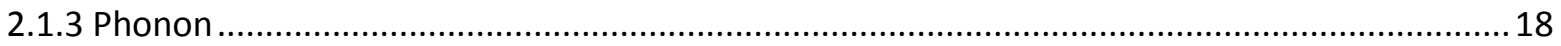

2.1.4 Optical Absorption in Semiconductors ............................................................................. 19

2.2 Optical Background for Solid-State Material Characterization...................................................... 21

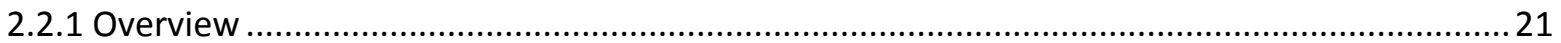

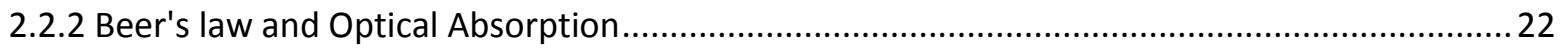

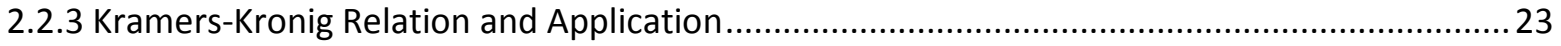

2.3 Electrical and optical properties of a-Si, c-Si, and silicon nanoparticles.........................................26

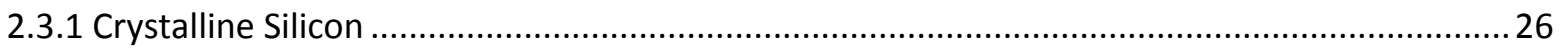

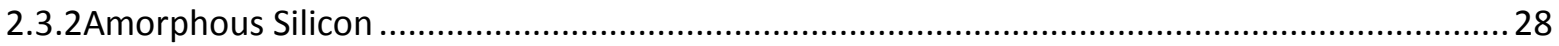

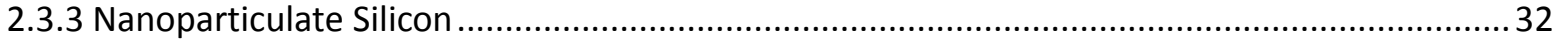

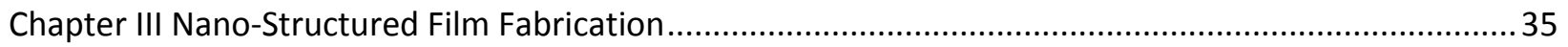

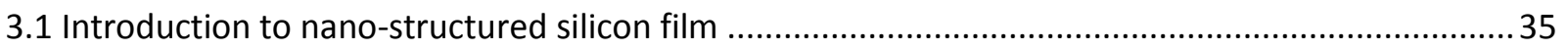

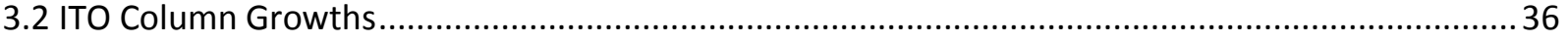

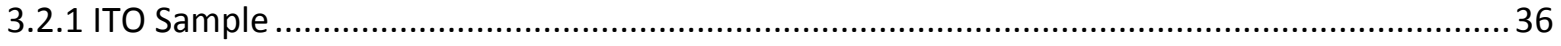

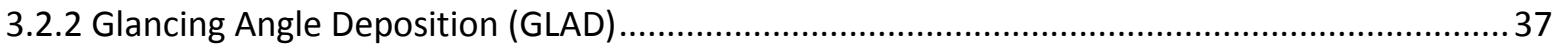


3.2.3 Vapour-liquid-solid growth glancing angle deposition ....................................................... 38

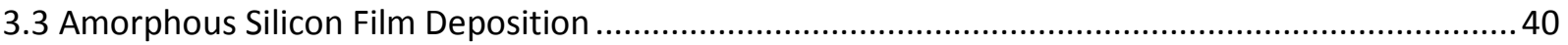

3.3.1 Overview of Low Pressure Chemical Vapor Deposition......................................................... 40

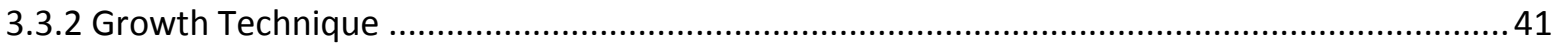

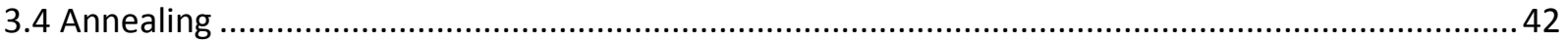

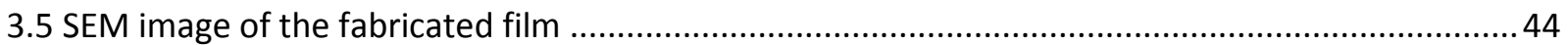

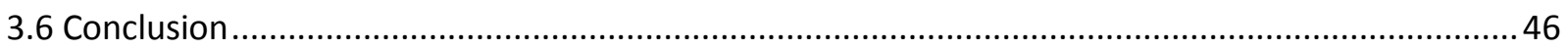

Chapter IV Experiment: Optical Characterization of Nanostructured Silicon Film ................................ 47

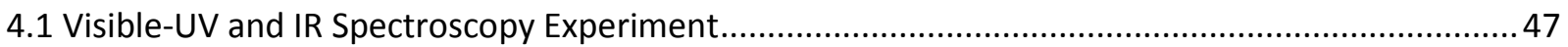

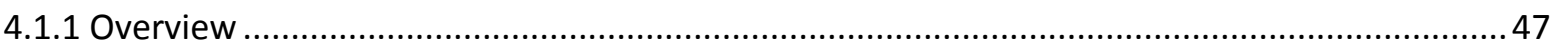

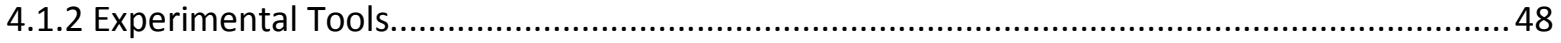

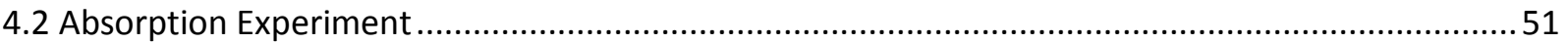

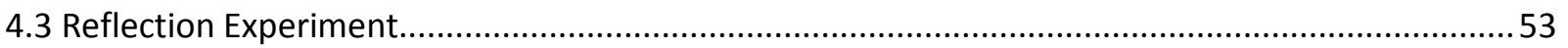

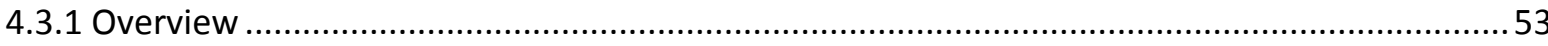

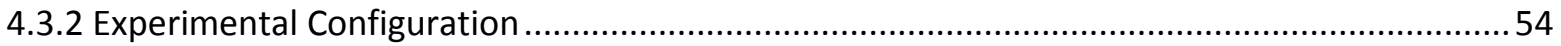

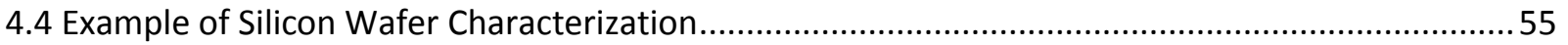

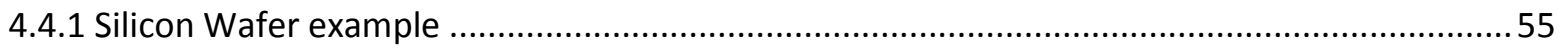

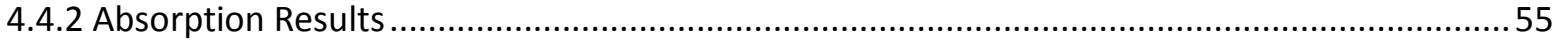

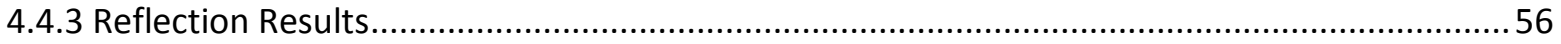

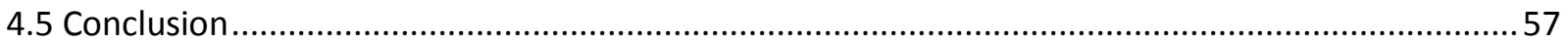

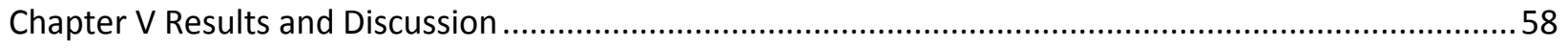

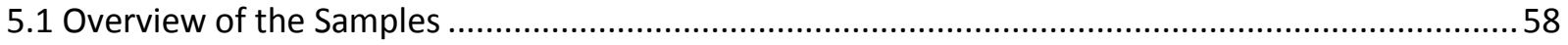

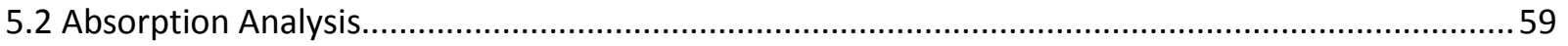

5.2.1 Amorphous silicon deposited on Glass, ITO Glass and Nano-structured ITO ..........................59

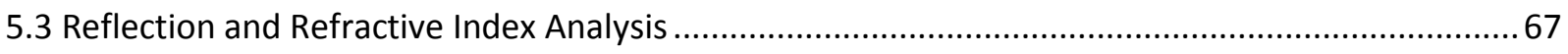

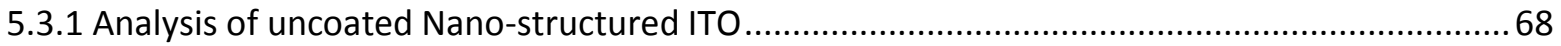

5.3.2 8nm thick amorphous silicon deposited on Nano-structured ITO.......................................... 72

5.3.3 8nm thick crystallized silicon deposited on Nano-structured ITO .......................................... 80

5.3.4 4nm thick amorphous silicon deposited on nano-structured ITO ............................................ 82

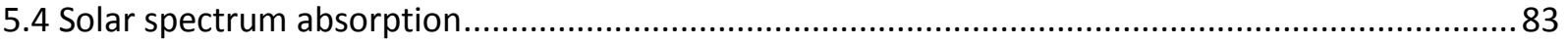

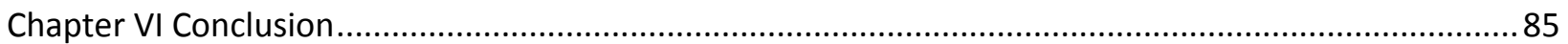




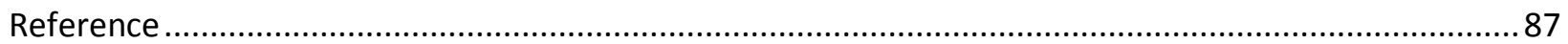

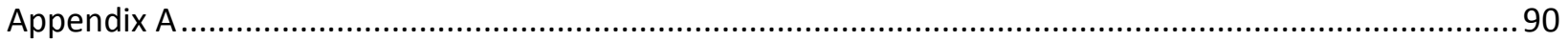




\section{List of Figures}

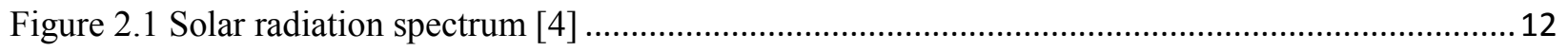

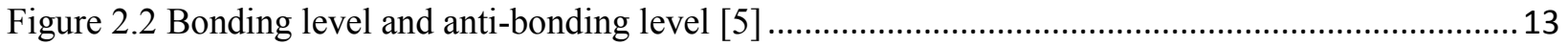

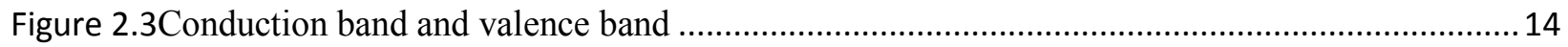

Figure 2.4 Schematic representation of transition from valence to conduction band [5] ....................... 15

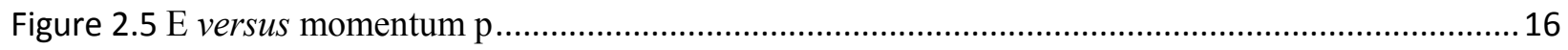

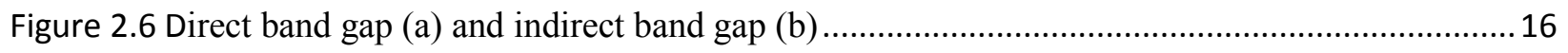

Figure 2.7 Longitudinal acoustic and optical phonon ...................................................................... 18

Figure 2.8 Traverse acoustic and optical phonon .............................................................................. 19

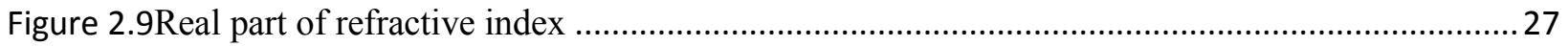

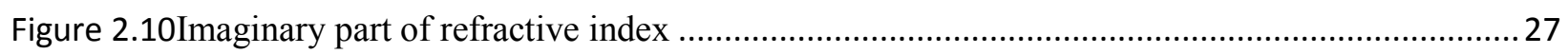

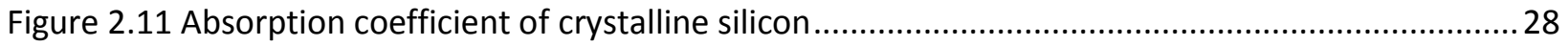

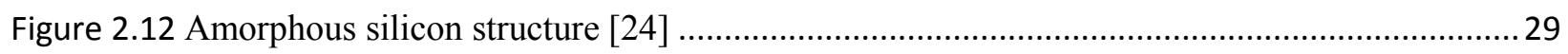

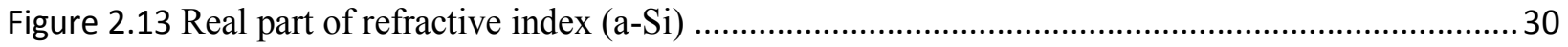

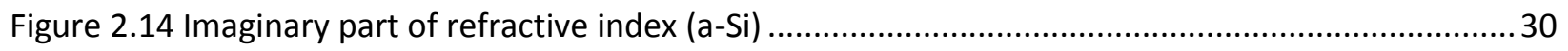

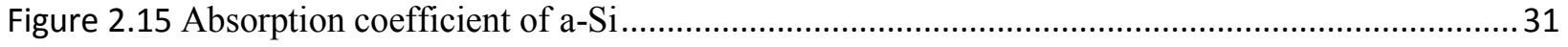

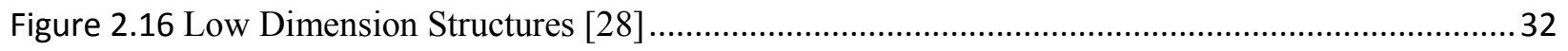

Figure 2.17 Density of states as a function of energy [28] ................................................................. 32

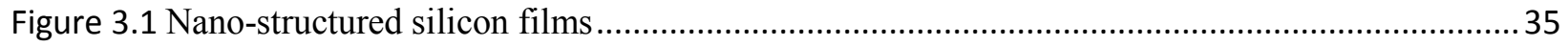

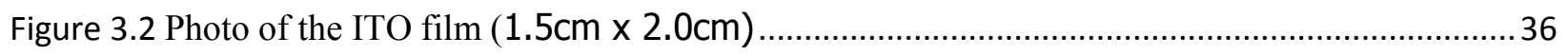

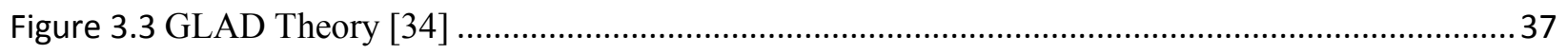

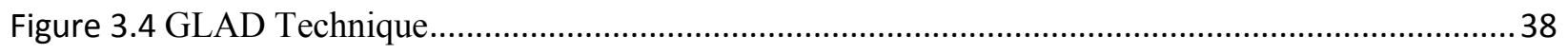

Figure 3.5 Qualitative parameter space for ITO planar, glancing angle deposition, vapour-liquid-solid

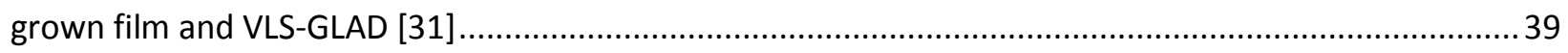

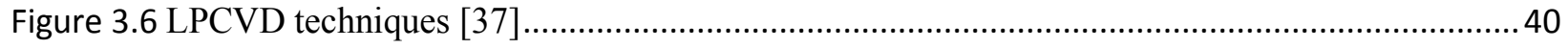

Figure 3.7 ITO deposited with an amorphous Silicon film .................................................................. 41

Figure 3.8 Comparison of roughness of a-Si annealed at different temperatures .................................... 42

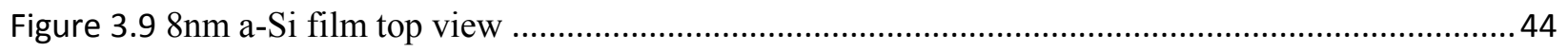

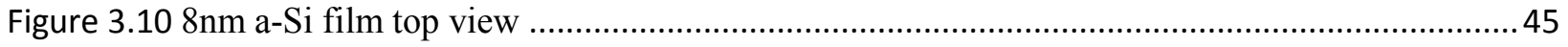

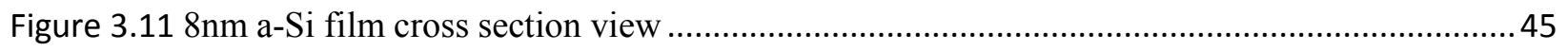

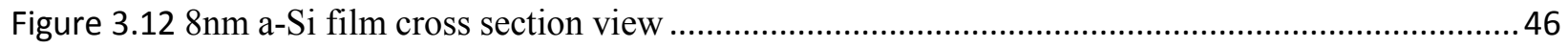

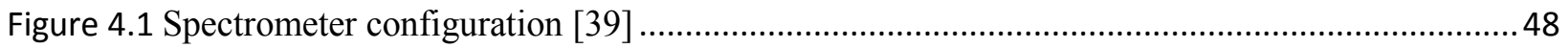

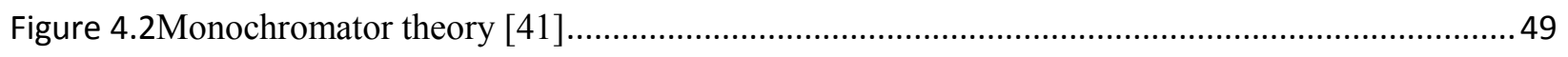

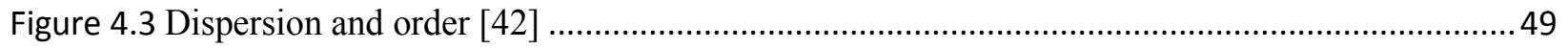


Figure 4.4 Photodiode sensitivity .50

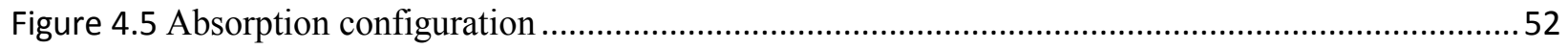

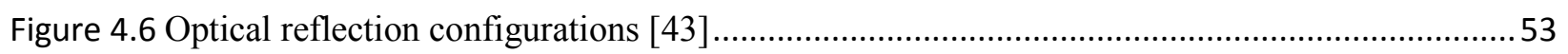

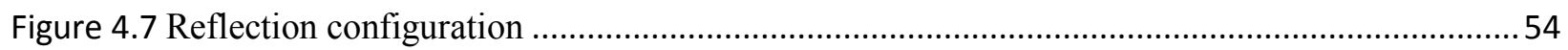

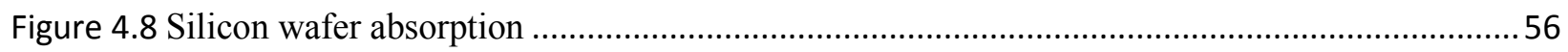

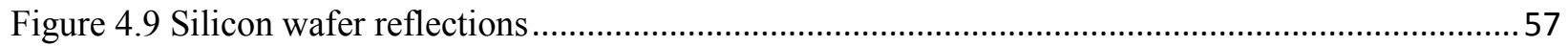

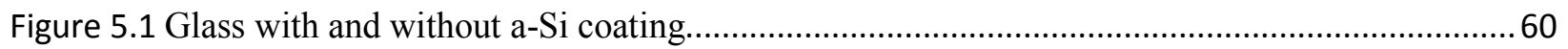

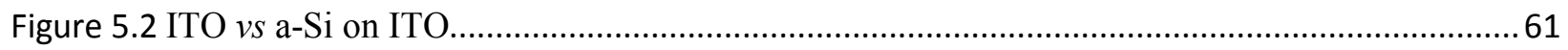

Figure 5. 3 Optical absorption of nano-structured ITO vs a-Si on nano-structured ITO ...........................62

Figure 5.4 Absorption of a-Si on different substrate (Glass: red curve; ITO: black curve; Nano-structured

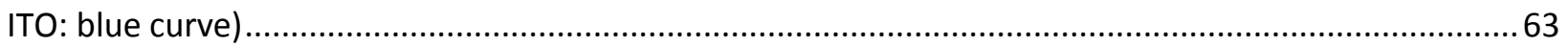

Figure 5.5: Absorption of 4nm, $8 \mathrm{~nm}$ amorphous film and ITO sample ( $4 \mathrm{~nm}$ film: blue curve; Nano-

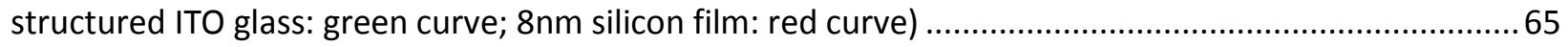

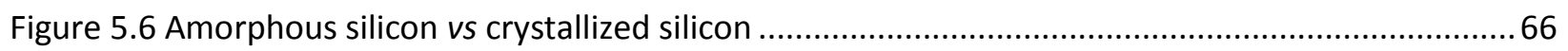

Figure 5.7Reflectance of uncoated nanostructured ITO (Si detector) ....................................................68

Figure 5.8 Reflectance of uncoated nanostructured ITO (Ge detector) ..................................................6

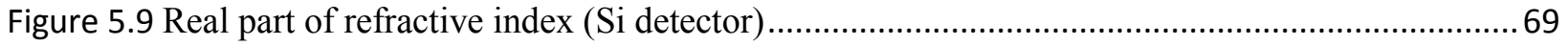

Figure 5.10Real part of refractive index (Ge detector) ........................................................................69

Figure 5.11 Imaginary part of refractive Index (Si detector) ................................................................ 70

Figure 5.12 Imaginary part of refractive index (Ge detector) ............................................................... 71

Figure 5.13 Reflection of 8nm a-Si film for 400-1100nm wavelengths ................................................. 72

Figure 5.14Reflection of $8 \mathrm{~nm}$ a-Si film for $1100-1800 \mathrm{~nm}$ wavelengths ................................................ 73

Figure 5.15 The real part of the refractive index 400-1100nm ............................................................ 76

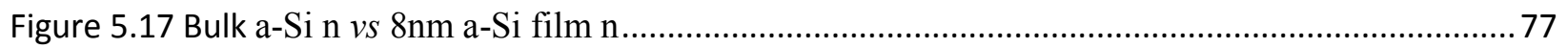

Figure 5.18 The imaginary part of refractive index $400-1100 \mathrm{~nm}$........................................................ 78

Figure 5.19 The imaginary part of refractive index $700-1800 \mathrm{~nm}$........................................................ 78

Figure 5.20 Calculated absorption coefficient (Si detector)................................................................... 79

Figure 5.21 Calculated absorption coefficient (Ge detector).............................................................. 80

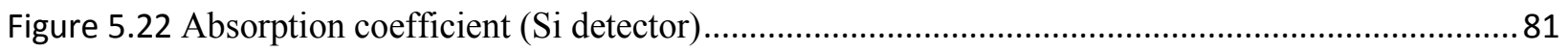

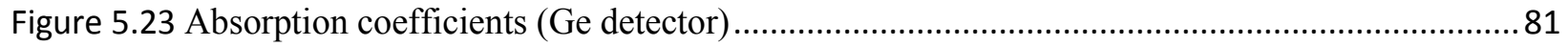

Figure 5.24 Absorption coefficient (Si detector) ........................................................................... 82

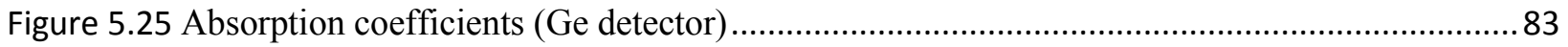




\section{Chapter I Introduction}

\subsection{Overview}

Silicon thin film solar cells are promising candidates for use in photovoltaic applications, owing to their advantages of low cost, material abundance and mature processing technology. However, the c-Si is an indirect gap material and so the optical absorption is low compared to many other solar cell materials. Thus, an efficient c-Si solar cell always comes with a thick layer. Also, the bandgap of c-Si and a-Si:H sit at $1140 \mathrm{~nm}$ and $700 \mathrm{~nm}$ which are much shorter than the solar spectrum (spectral irradiance is still at $0.3 \mathrm{~W} \mathrm{~m}^{-2} \mathrm{~nm}^{-1}$ even at1500 nm). Hydrogenated amorphous silicon (a-Si:H) thin films can provide much higher absorption coefficient as it is a direct bandgap semiconductor and a wider bandgap than crystalline silicon, but the welldocumented Stabler-Wronski degradation limits the efficiency of these devices. Recently, onedimensional nanostructure arrays, such as nanopillars, nanowire and nano holes, have proved to enhance the anti-reflection and broadband absorption [1][2][3]. All of these improvements are mainly due to a gradient in effective refractive index from the top of the structure to the substrate. Therefore, nanosructuring can shift the bandgap of the c-Si films to better match the solar spectrum by changing the refractive index. 


\subsection{Objective}

In this thesis, the main goal is to determine if the nanostructured Si film can modify bandgap to better match the solar spectrum. Also, another goal is to increase optical absorption of c-Si to have the advantage of a-Si:H without degradation. Last but not least, a bandgap measurement system needs to be set up to characterize the nanostructured a-Si film.

A new nanostructured a-Si silicon film was introduced in order to modify the bandgap and absorption of Si material. The nanostructured film was fabricated by depositing an ultra-thin (4 to $8 \mathrm{~nm}$ ) a-Si film over a nanostructured ITO substrate. The nanostructured ITO substrate was produced by the Glancing Angle Deposition research group in University of Alberta and the a-Si thin film was deposited in the fabrication lab of CarletonUniversity. The a-Si was crystallized by annealing at $600 \mathrm{C}$.

\subsection{Thesis Outline}

This thesis begins with a background chapter dealing with solid-state physic and the optical properties of semiconductors. In chapter II, the properties of semiconductor nanoparticlesare also discussed. The bandgap of the nanoparticulate material alters as the size of the nanoparticles changes. This effect can be used to shift the bandgap of a nanostructured semiconductor material.

In Chapter III, a nanostructured silicon film is proposed and sample fabrication described. 
ChapterIV reviews the optical methods for transparent thin film characterization. Both absorption and reflection experiment are discussed in this chapter.

In Chapter V, the absorption and the reflection measurements on the nanostructured silicon film are described and results given. The bandgap of the nanostructured silicon film was determined by using both absorption analysis and reflection analysis.

Finally, Chapter VI presents conclusions and suggestions for future work. 


\section{Chapter II Background}

\subsection{Energy bands in Solar Cell}

In a semiconductor solar cell, the optical absorption varies with the wavelengths. Also, the solar radiation spectrum in Fig.2.1 indicates that solar energy peaks at $485 \mathrm{~nm}$. As a result, we can increase the solar cell efficiency by shifting the energy band up and down to match the solar radiation spectrum. In general, it all starts from solid states physics.

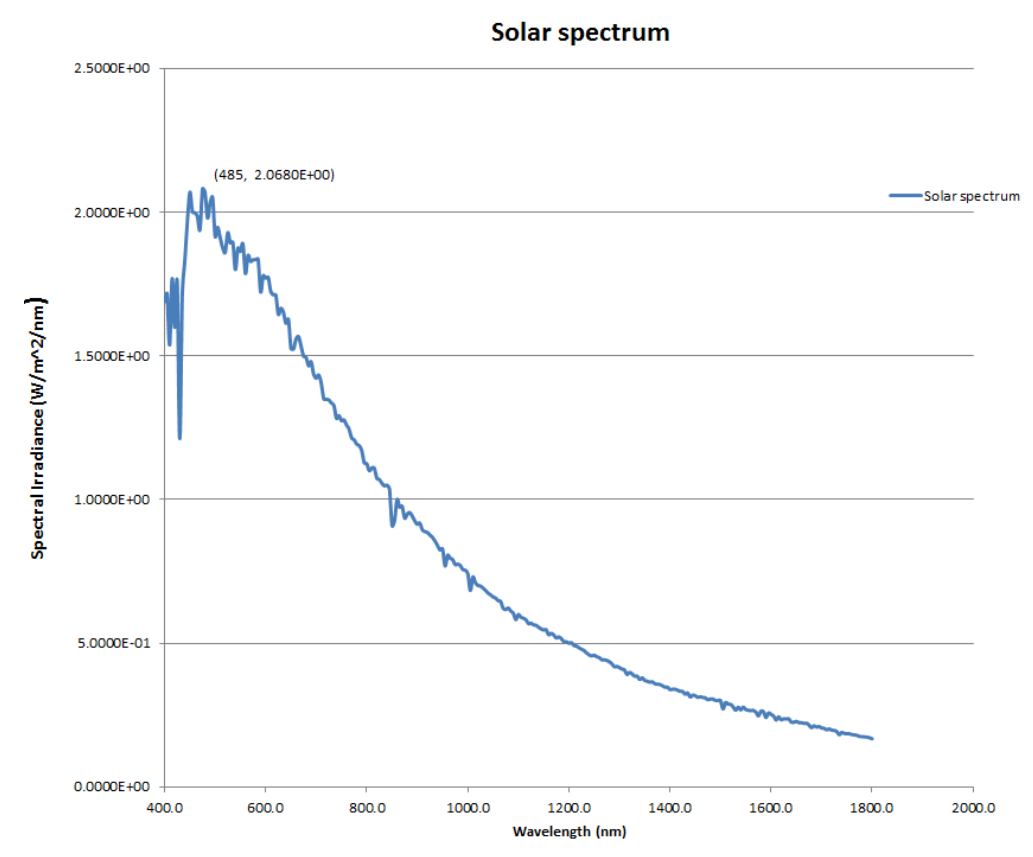

Figure 2.1Solar radiation spectrum [4]

\subsection{Solid States Physics Overview}

Solid states physics is a branch of condensed matter physics; it studies how the large-scale properties of solid materials result from their atomic-scale properties. As the focus point is on 
solid, it is important to understand how the solid material bonds and how an energy band formed in a semiconductor material.

\subsubsection{Introduction to the Energy Band}

Understanding energy bands in a semiconductor begins by considering the chemical bonds between atoms in crystal. A chemical bond may be formed either by sharing of electrons or by transfer of electrons between atoms. When two atoms combine to form a molecule, each valence electron energy level in the isolated atom contributes an energy level to the molecule. For example, when two hydrogen atoms combine to form a molecule, the 1s energy levels of the original atoms combine to form two molecular orbitals, one with lower energy and the other higher. In general Fig. 2.2 illustrates that no matter how many atoms and bonds one has in a molecule, half of the molecular orbitals are lowered in energy relative to the sum of the energies of the isolated atomic orbitals, and are referred to as the bonding orbitals. The other half of the molecular orbitals are raised in energy relative to the sum of the energies of the isolated atomic orbitals and are named anti-bonding orbitals.

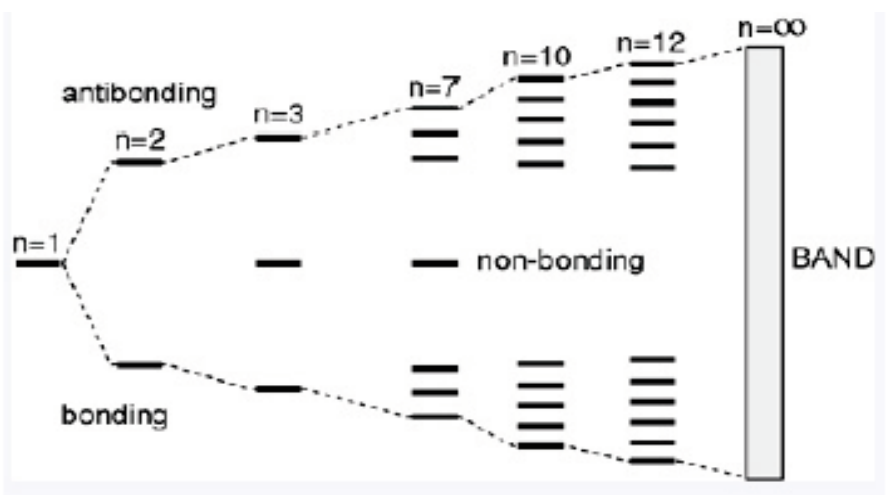

Figure 2.2Bonding level and anti-bonding level[5] 
When the number of atoms becomes very large (as in the case of semiconductor crystal), the molecular orbital energy levels become effectively continuous, giving rise to energy bands. The bonding orbitals form the valence band, which is completely occupied by electrons at absolute zero while the anti-bonding levels form the conduction band which is completely empty at absolute zero [5]. Figure 2.3 indicates the energy difference between the conduction band and the valence band. This energy difference is called the band gap.

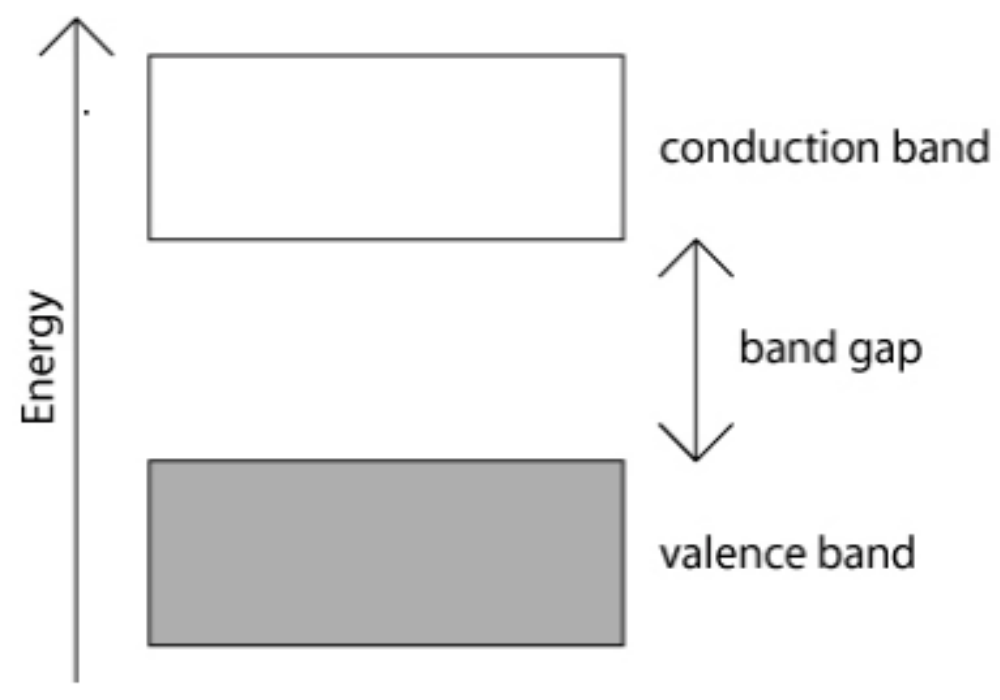

Figure 2.3Conduction band and valence band

This gap energy plays an important role in solid state physics. If the crystal absorbs more than the band gap energyfrom a photon or phonon,it will free an electron from the bonds so that it can move about the crystal lattice(Figure 2.4). This means that free electrons have larger energy than those electrons in the bonds. We can therefore give the bandgap energy a broad definition, the bandgap energy is the minimum energy required to liberate an electron[6]. 


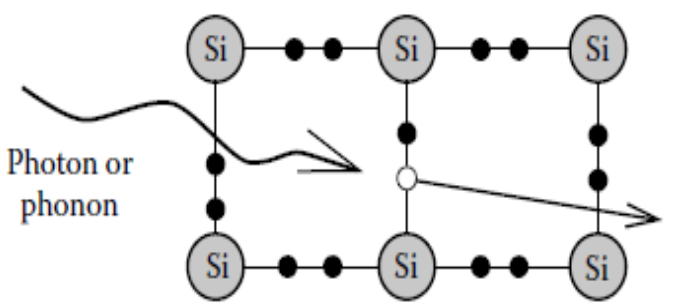

Figure 2.4Schematic representation of transition from valence to conduction band [5] An electron that hasthis minimum amount of energy must have a potential energy equal to the gap energy. Also, there are electrons that have more than this minimum energy, and these electrons have not only potential energy but also kinetic energy. Thus, we can write the total energy of an electron as:

$$
\mathrm{E}=\text { Potential Energy }+ \text { Kinetic Energy }=\mathrm{E}_{\mathrm{g}}+\frac{1}{2} \mathrm{~m}_{\mathrm{e}} \mathrm{v}^{2}(2.1)
$$

Where potential energy equals the gap energy $E_{g}$ and $m_{e}$ denotes an effective mass for an electron in the conduction band.

If we use the momentum $\mathrm{p}=\mathrm{m}_{\mathrm{e}} \mathrm{v}$, we can rewrite the relation as

$$
E=E_{g}+\frac{p^{2}}{2 m_{e}}
$$

Near the band edge,the energy E versus momentum $\mathrm{p}$ curve generally has a parabolic shape as shown in Figure 2.5. The upper curve and lower curve are conduction band and valence band respectively. If the electron receives just enough energy to travel from conduction band to valence band, it does not have enough energy to be moving and $p=0$. This energy diagram is referred as the band diagram[7]. 


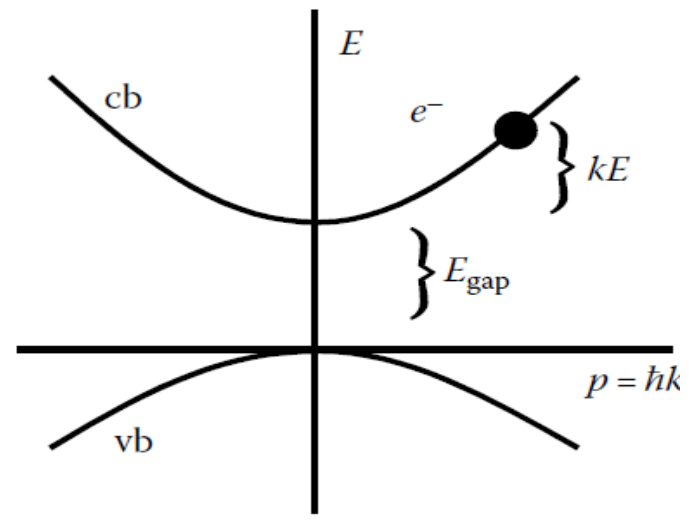

Figure $2.5 \mathrm{E}$ versus momentum $\mathrm{p}$

\subsubsection{Direct band gap and Indirect band gap}

As we discussed before, the band gap represents the minimum energy difference between the top of the valence band and the bottom of the conduction band. There are two types of band gap, the direct band gap as shown in Fig. 2.5 a) and the indirect band gap as shown in Fig. 2.5 b).
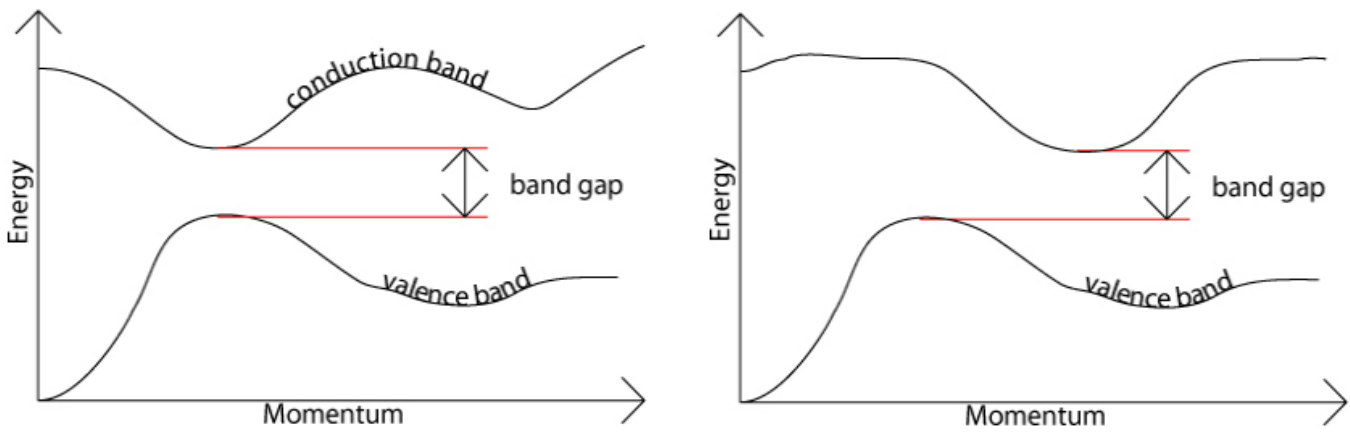

(a) (b)

Figure 2.6 Direct band gap (a) and indirect band gap (b) 
In a direct band gap material, the highest energy of the valence band and the lowest energy of conduction band stay at the same momentum value (Fig. 2.6 a). Examplesof direct band gap materials are GaAs and $\mathrm{InP}[8]$.

In the indirect band gap material,in contrast,the highest energy of the valence band and the lowest energy of conduction band occur at a different momentum (Fig. 2.6 b). Crystalline silicon, germanium and $\mathrm{GaP}$ are indirect band gap materials.

The difference between the two types of material is most important in optical devices. As has been mentioned in the previous section, a photon can provide the energy to produce an electronhole pair.

Each photon of energy $E$ has momentum:

$$
\mathrm{p}=\frac{\mathrm{E}}{\mathrm{c}}
$$

Where $c$ is the velocity of light. An optical photon has energy of the order of $10^{-19} \mathrm{~J}$, and also $c=3 \times 10^{8} \mathrm{~m}-\mathrm{s}^{-1}$, thus a typical photon has a momentum of:

$$
\mathrm{p}=\frac{10^{-19} \mathrm{~J}}{3 \times 10^{8} \mathrm{~ms}^{-1}}=0.333 * 10^{-27} \frac{\mathrm{J} * \mathrm{~s}}{\mathrm{~m}}
$$

An optical photon therefore has a very small amount of momentum. In the direct band gap material, a photon of energy $E_{g}$, can produce an electron-hole pair quite easily since the electron does not need to be given very much momentum. However, in indirect band gap material, an electron must also undergo a significant change in its momentum for a photon of energy $E_{g}$ to produce an electron-hole pair. The optical photon does not have enough momentum to allow the transition from conduction band to valence band itself. But, this can still occur when the electron interacts not only with the photon to gain energy, but also with a lattice vibration called a phonon in order to either gain or lose momentum. The indirect process proceeds at a much slower rate, as 
it requires three entities to intersect in order to proceed: an electron, a photon and a phonon. In contrast, direct processes only need an electron and a photon and are much faster.

The same principle applies to recombination of electrons and holes to produce photons. The recombination process is much more efficient for a direct band gap material than for an indirect band gap material, where the process must be mediated by a phonon.

\subsubsection{Phonon}

When heated, the atoms in a crystal can oscillate at specific frequencies. The bonds between the each atom in a crystal can be modelled as springs. When one of the atoms gets pushed or pulled, it creates a wave or phonon traveling through the crystal.

There are generally two types of phonon as shown in Fig. 2.7. In the top one, each adjacent atom moves in the same phase. It is called the acoustic phonon. In the bottom one, two adjacent phonon moves out of phase and is named an optical phonon. Acoustic phonon corresponds to intercellular interaction while optical phonon represents the intracellular interactions [9].

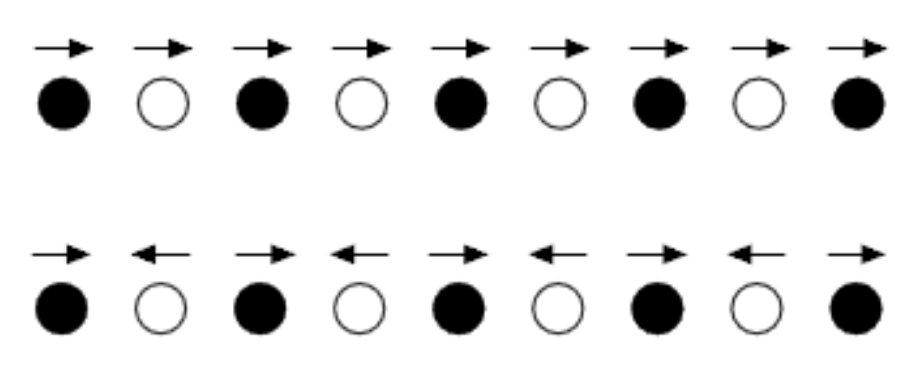

Figure 2.7Longitudinal acoustic and optical phonon

Also, the phonon can also vibrate through traverse direction as shown in Figure 2.8. 


\section{$\varphi \cdot b \cdot \varphi \cdot$

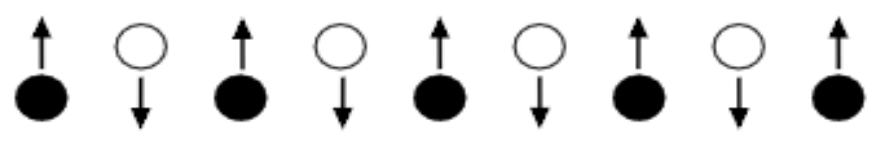

Figure 2.8Traverse acoustic and optical phonon

\subsubsection{Optical Absorption in Semiconductors}

In semiconductor material, there are several optical absorption processes: the fundamental absorption, exciton absorption, impurity absorption and free carrier absorption.

\subsubsection{Fundamental absorption}

The fundamental absorption is the excitation of electrons from valence band to conduction band. However, this absorption only occurs when the photon has energy greater than the bandgap energy. The energy or wavelength at which the fundamental absorption starts is called the absorption edge [10].

\subsubsection{Exciton Absorption}

In a semiconductor, exciton absorption occurs when an electron in the valence band absorbs almost enough energy to be excited to the conduction band, but electrons and holes produced by photon are bound together [11]. 


\subsubsection{Impurity absorption}

Impurity absorption occurs when an electron absorbs a photon that excites it from the valence band to an empty impurity atom or from an occupied impurity to the conduction band [12]. As silicon is usually quite pure material, impurity absorption is usually rare.

\subsubsection{Free Carrier Absorption}

This mechanism involves the absorption of a photon by interaction with a free carrier within a band, which is consequently raised to a higher energy. The transition of the carrier to a higher energy within the same valley must conserve momentum. This momentum change is provided by phonons. Free carrier absorption usually affects the long wavelength region with a monotonic increase in absorption with a wavelength dependence of the form $\lambda^{p}$, where $p$ ranges from 1.5 to 3.5[13]. The value of $\mathrm{p}$ depends on the nature of momentum conserving scattering. The absorption coefficient due to the free carrier absorption is often modeled as:

$$
\alpha=\frac{\mathrm{Nq}^{2} \lambda^{2}}{4 \pi^{2} \mathrm{~m}^{*} \mathrm{n}_{\mathrm{r}} \mathrm{c}^{3} \epsilon_{0}}\left\langle\frac{1}{\tau}\right\rangle
$$

Where $\mathrm{N}$ is the free carrier concentration, $\mathrm{n}_{\mathrm{r}}$ is the refractive index of the semiconductor, and $\left\langle\frac{1}{\tau}\right\rangle$ is the average value of the inverse of the relaxation time of the scattering process. 


\subsection{Optical Background for Solid-State Material Characterization}

\subsubsection{Overview}

Semiconductors are transparent to photons whose energies lie below their band gap and are strongly absorbing for photons whose energies exceed the band gap energy. Band-to-band absorption involves excitation of an electron from the valence band to the conduction band, resulting in creation of an electron-hole pair. There are several methods to determine the bandgap of a semiconductor. However, most of them are based on determining an edge in optical absorption. This can be observed by analyzing the absorption coefficient.

The band gap of a semiconductor can be determined by observing the transmission or absorption of light of various wavelengths. In section 2.2.2, Beer's law is introduced to describe how to obtain the bandgap by only measuring the transmittance.

The band gap can also be determined by finding the refractive index of the material at various wavelengths of light. The refractive index is in general complex

$$
\tilde{\mathrm{n}}=\mathrm{n}+\mathrm{ik} \quad(2.5 \mathrm{a})
$$

Where $\mathrm{n}$ is the real part of the index and $\mathrm{k}$ is the extinction coefficient. The number $\mathrm{k}$ indicates the amount of absorption loss when the wave propagates through the material [14]. The absorption coefficient $\alpha$ can be related to the extinction coefficient $\mathrm{k}$, by:

$$
\alpha=\frac{4 \pi \mathrm{k}}{\lambda}
$$

The absorption coefficient, $\alpha$, describes how far into a material light of a particular wavelength can penetrate before it is absorbed [15]. The inverse of the absorption coefficient $\alpha^{-1}$, is the 
average distance traveled by a photon before it is absorbed. The bandgap of a semiconductor material can be determined by analyzing the absorption coefficients a function of wavelength. In section 2.2.3, the Kramers-Kronig relation is used to calculate the absorption coefficient by measuring the reflections coefficients.

\subsubsection{Beer's law and Optical Absorption}

When light passes through a slab or film of material, its absorption is described by Beer's law as [16]:

$$
\mathrm{I}(\mathrm{z})=\mathrm{I}_{\mathrm{o}} \mathrm{e}^{-\alpha \mathrm{z}}
$$

Where,

$\mathrm{z}$ is the direction the wave propagates

$\mathrm{I}_{\mathrm{o}}$ is the intensity of the incident light

$\mathrm{I}(\mathrm{z})$ is the intensity of the transmitted light at $\mathrm{z}$

$\alpha$ is the absorption coefficient with the units of $1 / \mathrm{m}$

The absorption coefficient strongly depends on wavelength. $\alpha$ can be determined by measuring transmitted and incident light intensities at different wavelengths.

During the measurement, surface reflection will cause light loss. However, if the incident light is guided to normal incidence angle (90 degree), the reflection isclose to $40 \%$.It is also convenient to define transmittance as [17]:

$$
T(\lambda)=\frac{I(\lambda)}{I_{0}(\lambda)}
$$


The absorbance is then given by:

$$
\mathrm{A}=-\ln \mathrm{T} \quad(2.8)
$$

Equation 2.8, only considers the raw absorbed power. It does not take into account the thickness of the sample. This method does not give the actual absorption coefficient. But, in terms of determining bandgap, using the absorbance curve is sufficient.

\subsubsection{Kramers-Kronig Relation and Application}

\subsubsection{Kramers-Kronig Equations}

The Kramer-Kronig relations are equations that connect the real and imaginary parts of any analytic complex function. The equations for the real part and imaginary part are given as follows [18]:

$$
\begin{aligned}
& \epsilon_{1}(\omega)-1=\frac{2}{\pi} P \int_{0}^{\infty} \frac{\omega^{\prime} \epsilon_{2}\left(\omega^{\prime}\right)}{\omega^{\prime 2}-\omega^{2}} d \omega^{\prime} \\
& \epsilon_{2}(\omega)=-\frac{2 \omega}{\pi} P \int_{0}^{\infty} \frac{\epsilon_{1}\left(\omega^{\prime}\right)-1}{\omega^{\prime 2}-\omega^{2}} d \omega^{\prime}
\end{aligned}
$$

Where

P denotes the Cauchy principal value

$\epsilon_{1}(\omega)$ and $\epsilon_{2}(\omega)$ are the real and imaginary part of the complex number

$$
\epsilon(\omega)=\epsilon_{1}(\omega)+j \epsilon_{2}(\omega)(2.10)
$$


$\omega$ is the angular frequency and $\omega=2 \pi f$ and $f=\frac{c}{\lambda}$

The Kramers-Kronig relation is a mathematics solution to relate real and imaginary part of a complex number. It can be used to solve different kinds of problems. Given the real part, the imaginary part can be found, and vice versa. It is a very helpful in analysis of the optical constants of a solid-state material since the only input is the reflectance.

\subsubsection{Application in Refractive Index}

Kramers-Kronig analysis is the most commonly used method to obtain optical constants, such as $\mathrm{n}$ and $\mathrm{k}$. This method is based on the measurement of reflectance at normal incidence, over an energy or wavelength range, from a bulk material and the application of an integral transformation[19].

In this analysis, the reflectance $R(\omega)$ of a sample film is first measured over a certain wavelength range. The reflectance $R(\omega)$ is related to the phase shift $\theta(\omega)$ by the Fresnel equation. It is well known from Fresnel equation that, in any solid material, the complex reflectance is given as[20]:

$$
\tilde{\mathrm{r}}=\sqrt{\mathrm{R}(\omega)} \mathrm{e}^{\mathrm{j} \theta(\omega)}=\sqrt{\mathrm{R}(\omega)} \cos \theta(\omega)-\mathrm{j} \sqrt{\mathrm{R}(\omega)} \sin \theta(\omega)(2.11)
$$

Where

$R(\omega)$ is the reflectance and $\theta(\omega)$ is the phase shift caused by the reflection By taking logarithm of the above equation, one gets:

$$
\ln (\tilde{\mathrm{r}})=\ln (\sqrt{\mathrm{R}(\omega)})+\mathrm{j} \theta(\omega)(2.12)
$$


According to the Kramers-Kronig relation, the imaginary part and real part can be related as the following equation[21]:

$$
\theta(\omega)=-\frac{\omega}{\pi} P \int_{0}^{\infty} \frac{\ln (R(\omega))}{\omega^{\prime 2}-\omega^{2}} d \omega
$$

Also, when incident light with a frequency w reaches the surface of a film, the reflection can be expressed as[22]:

$$
r(\omega)=\frac{(n(\omega)-j k(\omega)-1)}{(n(\omega)-j k(\omega)+1)}(2.14)
$$

We relate equation 2.11 to equation 2.14 ,

$$
\sqrt{R(\omega)} \cos \theta(\omega)-j \sqrt{R(\omega)} \sin \theta(\omega)=\frac{(n(\omega)-j k(\omega)-1)}{(n(\omega)-j k(\omega)+1)}
$$

In equation 2.15 , equal the real part and the imaginary part in the left to the real part and imaginary part in the right side, respectively. One get:

$$
\frac{\left[\mathrm{n}^{2}(\omega)-1-\mathrm{k}^{2}(\omega)\right]}{\left.[\mathrm{n}(\omega)+1]^{2}+\mathrm{k}^{2}(\omega)\right]}=\sqrt{\mathrm{R}(\omega)} \cos \theta(\omega)
$$

And,

$$
\frac{2 \mathrm{nk}}{\left.[\mathrm{n}(\omega)+1]^{2}+\mathrm{k}^{2}(\omega)\right]}=\sqrt{\mathrm{R}(\omega)} \sin \theta(\omega)
$$

Solving $\mathrm{n}$ and $\mathrm{k}$ from equation 2.16 and 2.17 , one can obtain the optical constants $\mathrm{n}$ and $\mathrm{k}$ via the following equations:

$$
n(\omega)=\frac{(1-R(\omega))}{(1+R(\omega)-2 \sqrt{R(\omega)} \cos \theta(\omega))}
$$

and, 


$$
k(\omega)=\frac{-2 \sqrt{R(\omega)} \sin \theta}{1+R(\omega)-2 \sqrt{R(\omega)} \sin \theta}
$$

Now, if we solve for the phase shift $\theta$ from equation 2.13, we will have all the input variables needed for solving for $\mathrm{n}(\omega)$ and $\mathrm{k}(\omega)$ in equation 2.18 and equation 2.19 . The above equation can be written as a mathematic model in MATLAB-the program is given in Appendix A.

\subsection{Electrical and optical properties of a-Si, c-Si, and silicon nanoparticles}

Silicon is a group IV semiconductor material and it is the most commonly used semiconductor material as it forms the basis for integrated circuit chips and is the most widely used material in solar cells. Silicon can exist in either crystalline or amorphous forms. The two forms have distinct electronic and optical properties. Also, the electronic and optical properties of silicon crystallites of nano meter size will differ from bulk silicon. In the following section, the electrical and optical property of amorphous silicon, crystalline silicon and silicon nanoparticles will be discussed.

\subsubsection{CrystallineSilicon}

Crystalline silicon(c-Si) has the diamond lattice structure, with each $\mathrm{Si}$ atom sharing four covalent bonds with its neighbours. [23].

The real and imaginary components of the refractive index for crystalline silicon at 300 Kare shown in figure 2.9 and figure $2.10[24]$. 


\section{c-Si refractive index}

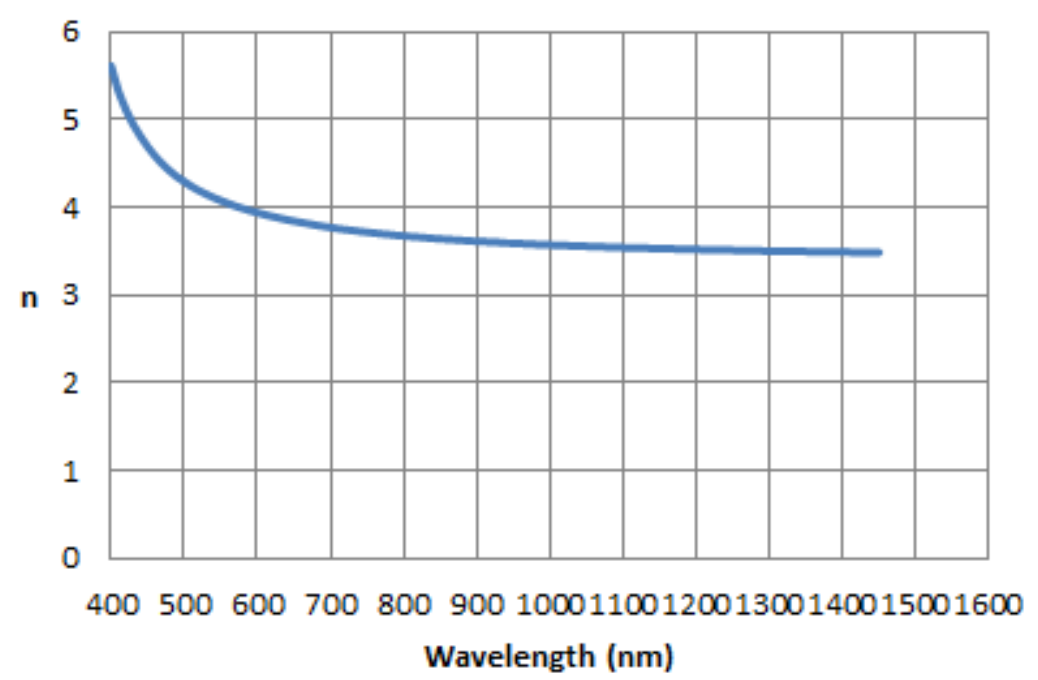

Figure 2.9Real part of refractive index

\section{c-Si extinction coefficient}

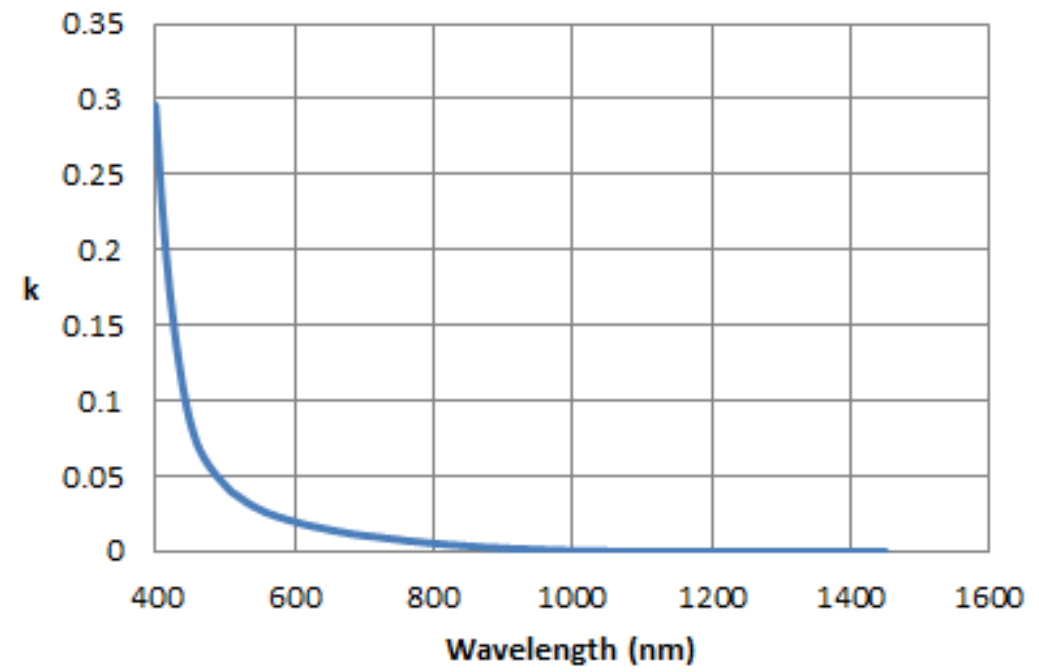

Figure 2.10Imaginary part of refractive index

The extinction is due to absorption and scattering. The extinction coefficient $k$ is related to the absorption coefficient by $\alpha=\frac{4 \pi k}{\lambda}$. Figure 2.11 showsthe c-Si absorption coefficient. Crystalline silicon is an indirect bandgap semiconductor so there is a long tail in absorption out to long 
wavelengths. Arbitrarily an absorption of $10^{0} \mathrm{~cm}^{-1}$ to define the bandgap edge gives the bandgap for c-Si of around 1140nm or $1.0875 \mathrm{eV}$ [24].

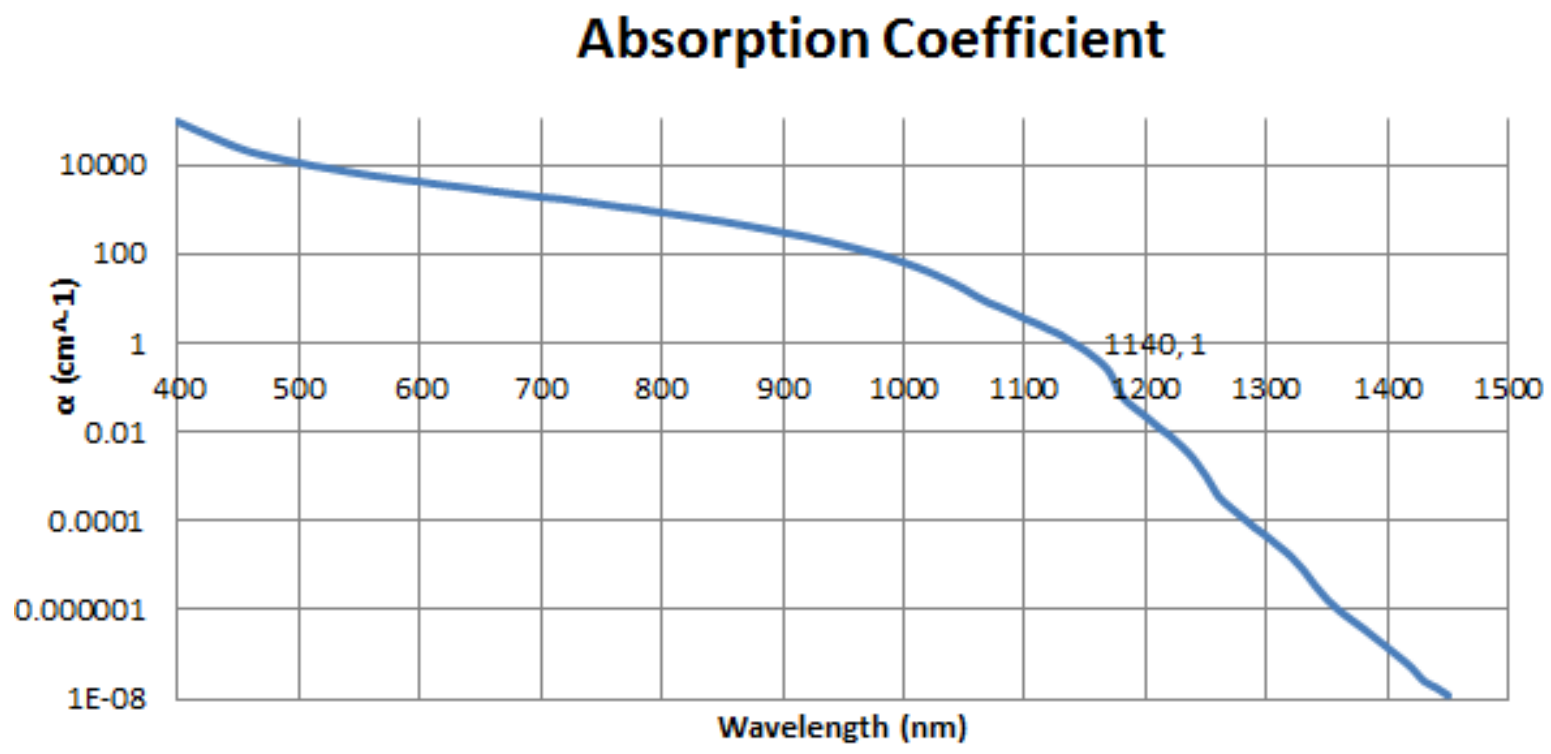

Figure 2.11Absorption coefficient of crystalline silicon

Crystalline silicon exhibits predictable and uniform behavior but because of the slow, high temperature manufacturing processes required, it is also the most expensive type of silicon.

\subsubsection{Amorphous Silicon}

In crystalline silicon the tetrahedral diamond structure is continued over a large range, forming a well-ordered lattice (crystal). In amorphous silicon (a-Si) this long-range order is not present and the atoms form a continuous random network. Not all the atoms within amorphous silicon are four-fold coordinated. Due to the disordered nature of the material, some atoms have a dangling bond as shown in Figure2.12. These dangling bonds are defects in the continuous random network, which cause undesired electrical behavior. However, a-Si can be passivized with atomic hydrogen(a-Si:H), which bonds to the dangling bonds and neutralizes this defect. 
Hydrogen passivized amorphous silicon has a sufficiently low density of defects to be used within devices. Amorphous silicon can be deposited over large areas using chemical vapor deposition methods [25].

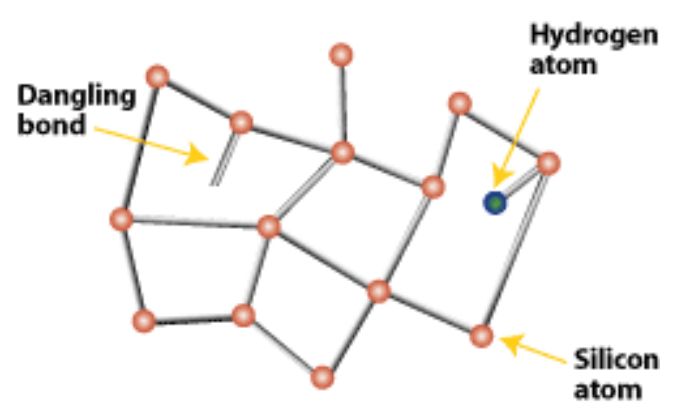

Figure 2.12Amorphous silicon structure [24]

The a-Si:H suffered from metastable changes due to the Staebler-Wronski effect. The dark conductivity and photoconductivity of the hydrogen passivated amorphous silicon can be significantly reduced with intense light illumination. This effect is reversible with annealing the silicon at hundred degrees Celsius for couple of hours. The disorder in the amorphous silicon structure appears play a major role in this effect. As a result, nanocrystalline silicon is a substitute material since it suffers less from the Staebler-Wronski effect. 
The real part and imaginary part of the refractive index for amorphous silicon at 300 Kare shown in figure 2.13 and figure $2.14[26]$.

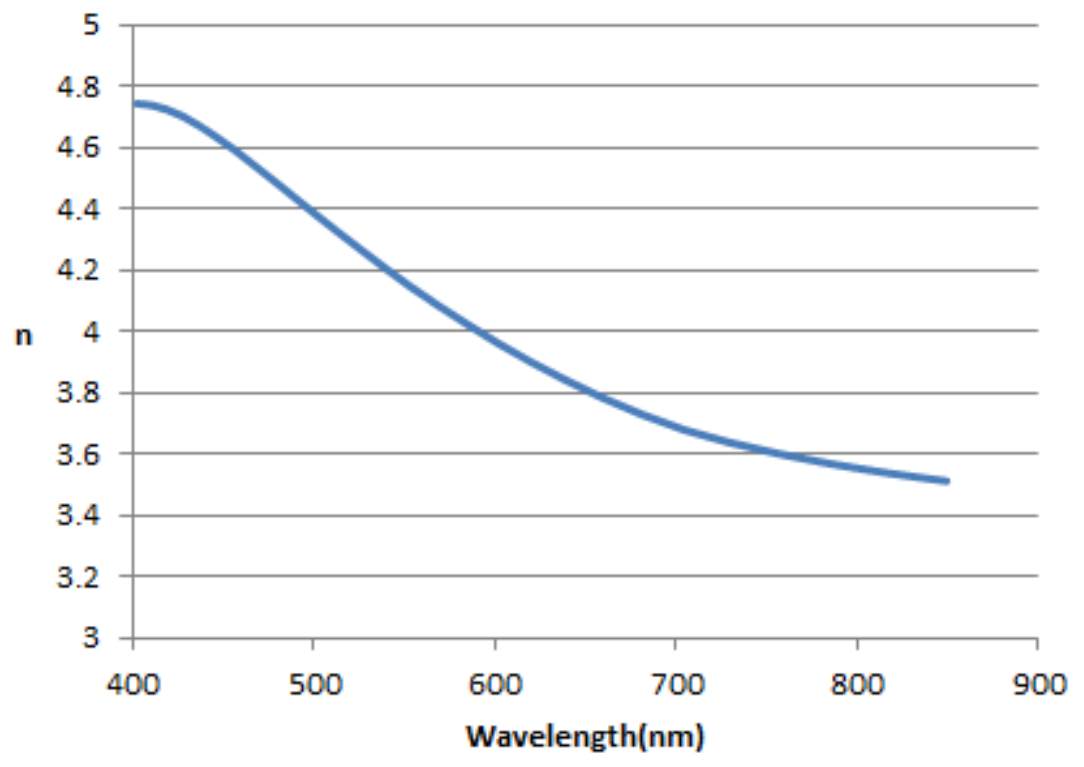

Figure 2.13Real part of refractive index (a-Si)

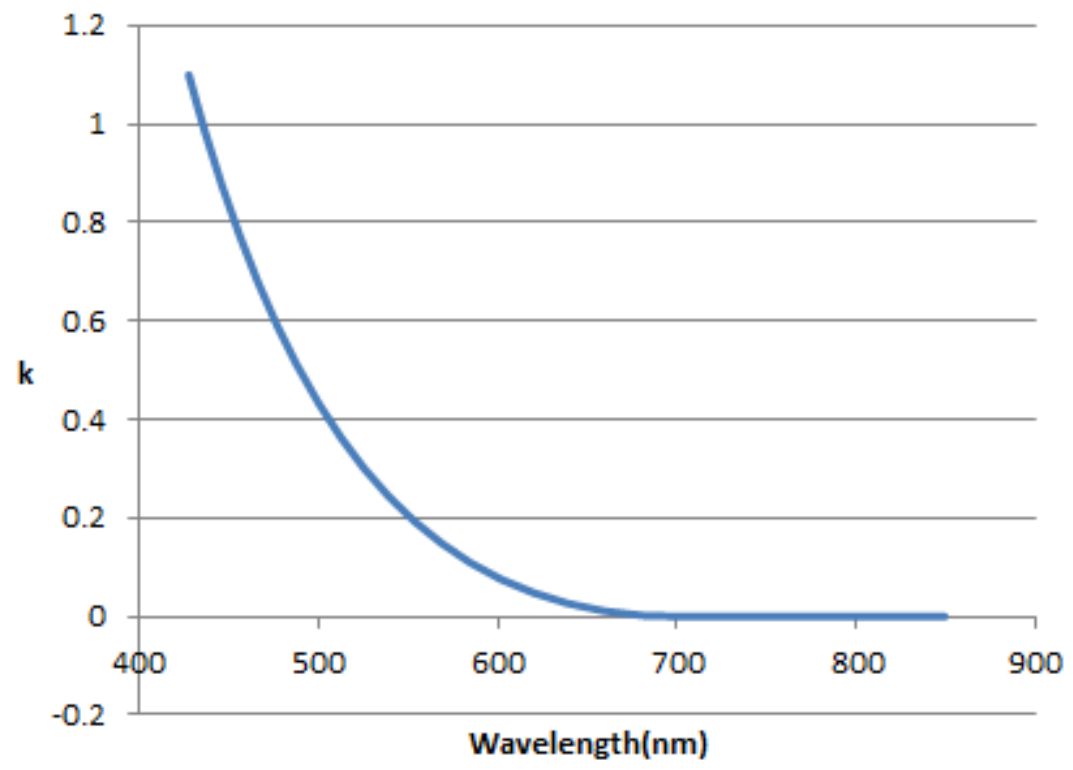

Figure 2.14 Imaginary part of refractive index (a-Si) 
Amorphous silicon becomes a direct bandgap semiconductor with a band gap of about $704.5 \mathrm{~nm}$ or $1.7611 \mathrm{eV}$ as shown in Figure 2.15.Also, the absorption is higher in a-Si compared to crystal silicon in Figure 2.11.

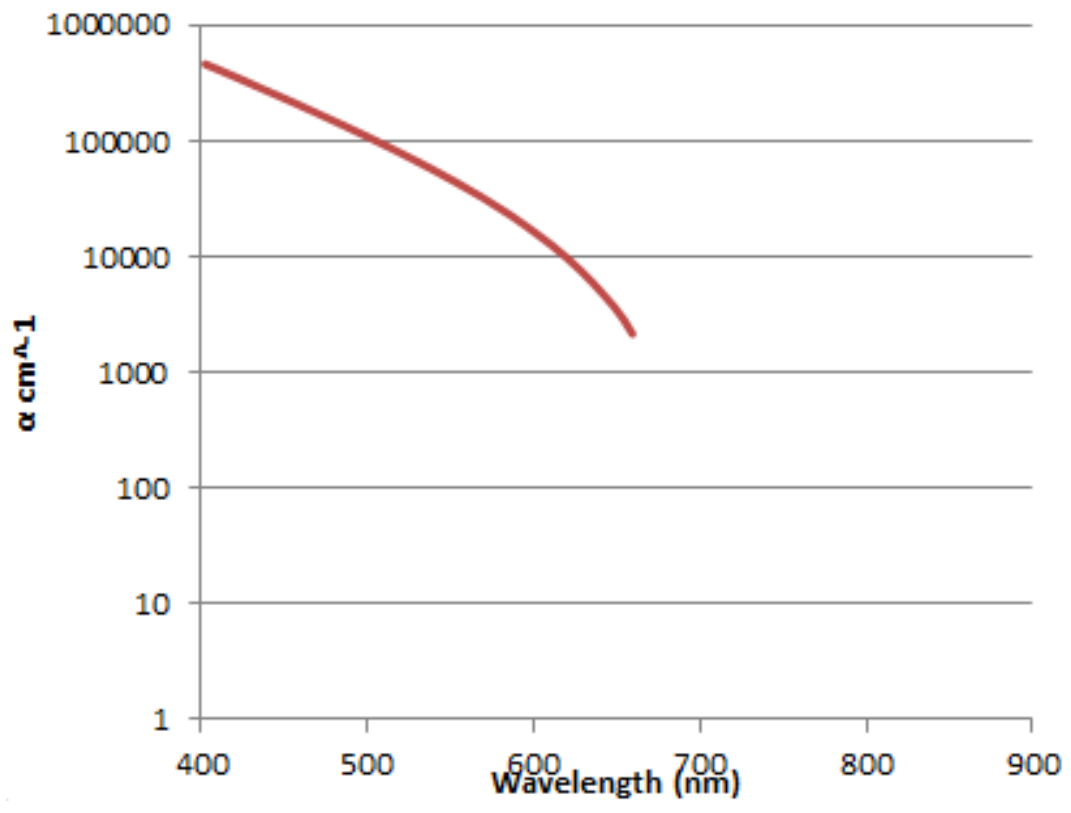

Figure 2.15Absorption coefficient of a-Si 


\subsubsection{Nanoparticulate Silicon}

Silicon nanoparticles are crystalline clusters containing approximately 500 to 2000 atoms corresponding to dimensions of roughly 1 to $100 \mathrm{~nm}$ [27].But, in order to observe the quantum mechanical properties, the dimension of the structure should be smaller than its Bohr radius. In silicon, the exciton Bohr radius is $\sim 4.5 \mathrm{~nm}[28]$. Thus, the silicon nanoparticles should be less than $4.5 \mathrm{~nm}$. In bulk silicon the electrons are free to move in 3 dimensions as shown in figure 2.16. Now suppose the bulk silicon is spatially confined in one direction. A thin silicon film will be obtained and it is known as 2Dquantum well. Taking this spatial confinement further with this quantum well, a 1D quantum wire will be created. Eventually, when a material is confined in all directions, a 0D structure is formed and it is also known as quantum dot [29].

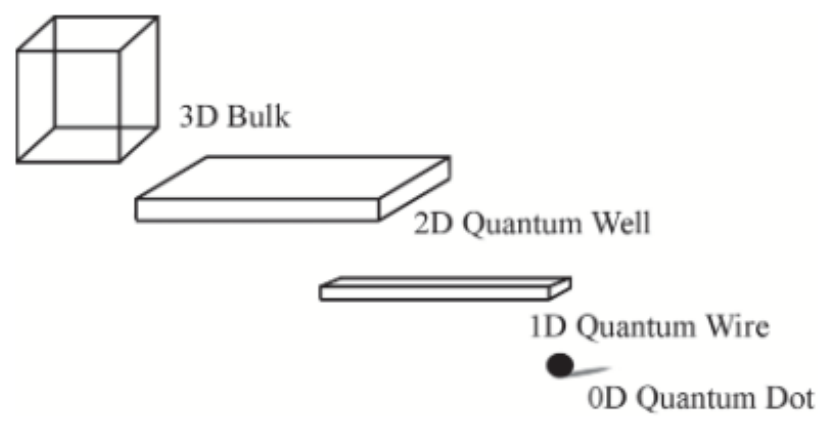

Figure 2.16Low Dimension Structures[28]

The quantum confinement of the electrons generates different densities states for each low dimension as shown in Figure 2.17.

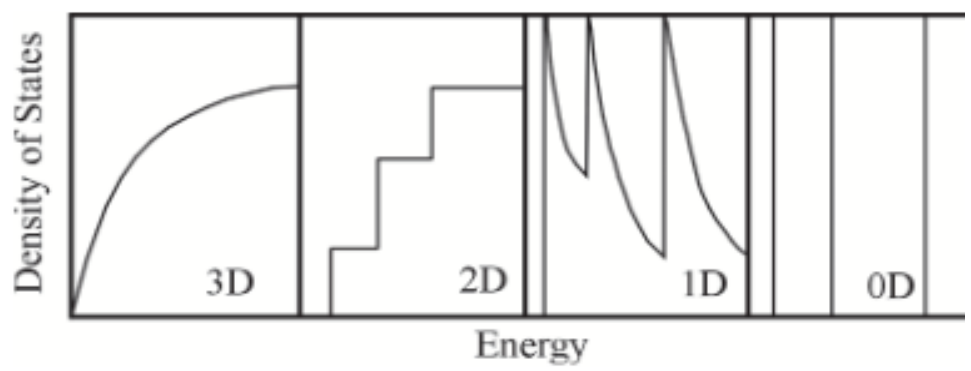

Figure 2.17Density of states as a function of energy [28] 
In the bulk or 3D, the carrier exists in nearly continuous bands. This is totally different from $0 \mathrm{D}$ structure where the carriers are restricted to a specific set of completely quantized energy states. The effect of confinement in the resulting energy states of the system can be obtained by solving for the eigen-energy of the Schrodinger wave equation for the carrier in a confined space. In a one-dimension structure, the energies allowed may be written as[30]:

$$
E_{n}=\frac{n^{2} \hbar^{2} \pi^{2}}{2 m L^{2}}(2.20)
$$

Where,

$m$ is the carrier effective mass;

$n$ is an integer $(1,2,3 \ldots \ldots .$.$) ;$

$\hbar$ is the reduced Plank constant;

$L$ is the confinement length.

According to equation 2.20, the energy levels are inverse proportional to the square of the confinement dimension $L$. As a result, the energy of the allowed states increases as the dimension decreases. The lowest possible energy for the particle (its zero-point energy) is found in state 1, which is given by:

$$
E_{1}=\frac{\hbar^{2} \pi^{2}}{2 m L^{2}}(2.21)
$$

Overall, the bandgap of semiconductor nanoparticles can be altered by increase or decrease the dimension of those particles. 


\subsection{Conclusion}

The bandgap of semiconductor is the energy required for absorption to happen. Shifting the bandgap of a semiconductor can alter the absorption performance.

Silicon is the most crucial semiconductor material for solar cell application. The bandgap of

nanoparticulate silicon shifts with its dimensions. With this feature, we can engineer the bandgap or the absorption of the silicon to match the solar spectrum. 


\section{Chapter III Nano-Structured Film Fabrication}

\subsection{Introduction to nano-structured silicon film}

A cross-section through the nano-structured silicon film examined here is shown in Figure 3.1 below. A glass substrate is coated with a planar indium tin oxide (ITO) film. ITO column or ITO nanowhisker[31] are grown on this film, and the column are coated with a thin layer of amorphous silicon that maybe annealed to form polycrystalline material. ITO is chosen as a template for the structure because it is both highly conductive and transparent. And therefore will not have a significant impact on light absorption in the silicon film.

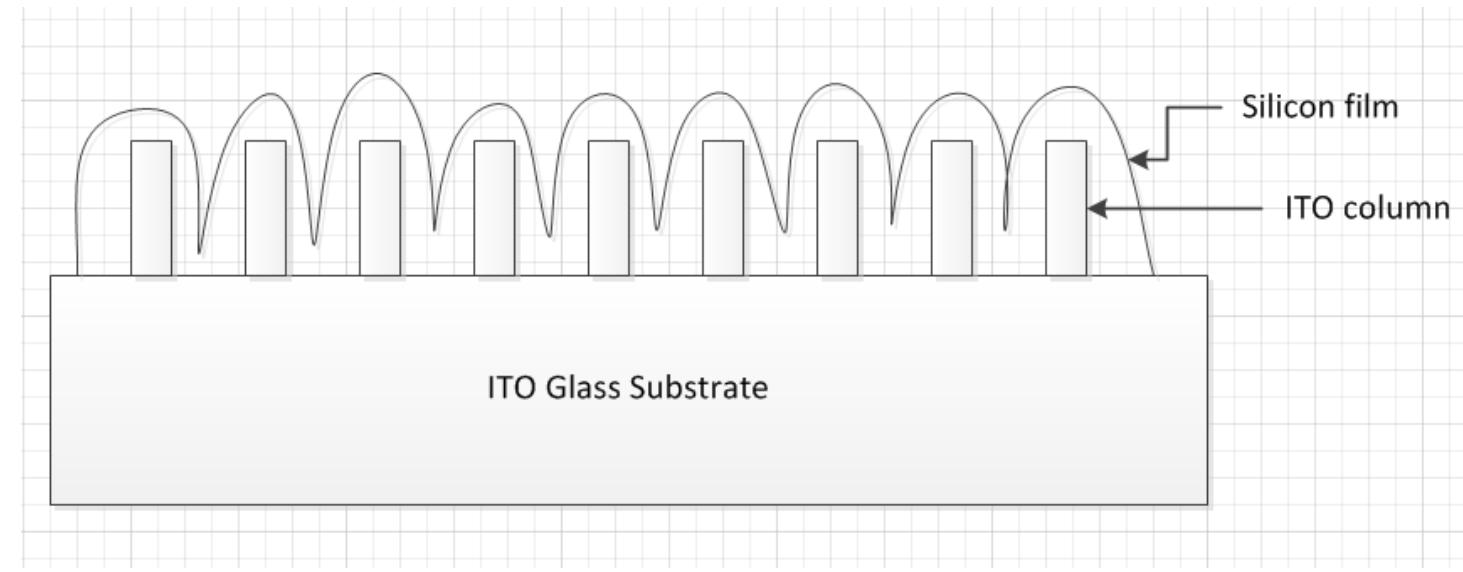

Figure 3.1Nano-structured silicon films

On top of the ITO substrate, there are ITO columns that are deposited by the vapour-liquid-solid Glancing Angle Deposition (VLS-GLAD) Technique. The grown ITO columns are tree like in structure. Every tree (ITO column)is about190nm in diameter and 400nm in height. These trees play a template role during the deposition of the amorphous silicon film. The silicon film deposited on top of these trees will automatically form the tree structured a-Si film. There are three steps to fabricate this film, the ITO column growth, the amorphous film growth and the annealing of the amorphous film. Each of the steps will be discussed in the following sections. 


\subsection{ITO Column Growths}

The "tree" like ITO nanostructure was grown by the Dr. Michael Brett and his VLS-GLAD Research Group. from University of Alberta.

\subsubsection{ITO Sample}

Indium-tin oxide is a transparent semiconducting oxide thin film. The band gap varies with composition and has been reported to be approximately3.75 eV [32]. Typically, the carrier concentration in ITO is around $10^{20}$ to $10^{21} \mathrm{~cm}^{-3}$ [33]. As a result, ITO has metal like electrical properties. The melting point of ITO is at $1730^{\circ} \mathrm{C}[34]$. Figure 3.2 shows a visible light photograph of an ITO film fabricated by the University of Alberta. It has a very high optical transmittance in the visible and near-infrared regions and high reflectance in the infrared region.

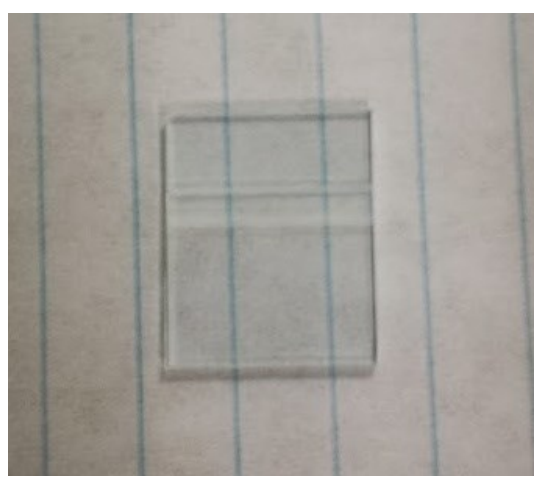

Figure 3.2Photoof the ITO film $(1.5 \mathrm{~cm} \times 2.0 \mathrm{~cm})$ 


\subsubsection{Glancing Angle Deposition (GLAD)}

The GLAD method is capable of fabricating ordered arrays of nanostructures. This method utilizes a vapor flux imping on a substrate from an oblique angle $\alpha$, as shown in Figure 3.3(a). This results in a deposited film showing a columnar morphology. Meanwhile, if the substrate is rotated around its normal direction, the morphology of the film is dramatically changed. Overall, the morphology of the column can be controlled by changing the substrate angular frequency and substrate position as shown in Figure 3.3(b) [35].

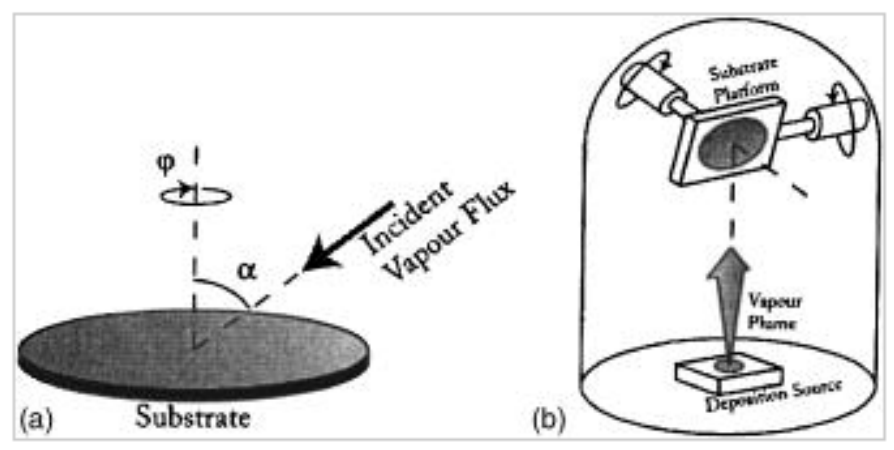

Figure 3.3GLAD Theory [34]

More specifically, when the gas flux reaches the surface of the substrate with an incident angleadefined in Figure 3.4(a), a nominally plannar film will be formed[36]. The arrival of the vapour flux and formation of film nuclei is a random process at this stage. The incident angle $\alpha$ controls the tilt of the columns and affects the degree of shadowing and thus the porosity of the film. This occurs when the small nuclei grow into columns, seen in Figure 3.4(b). The columns and shadows they cast will have a size distribution. As a results, some nuclei will screen neighboring nuclei from incoming gas flux and thus supress their growth as shown in Figure 3.4(c). 

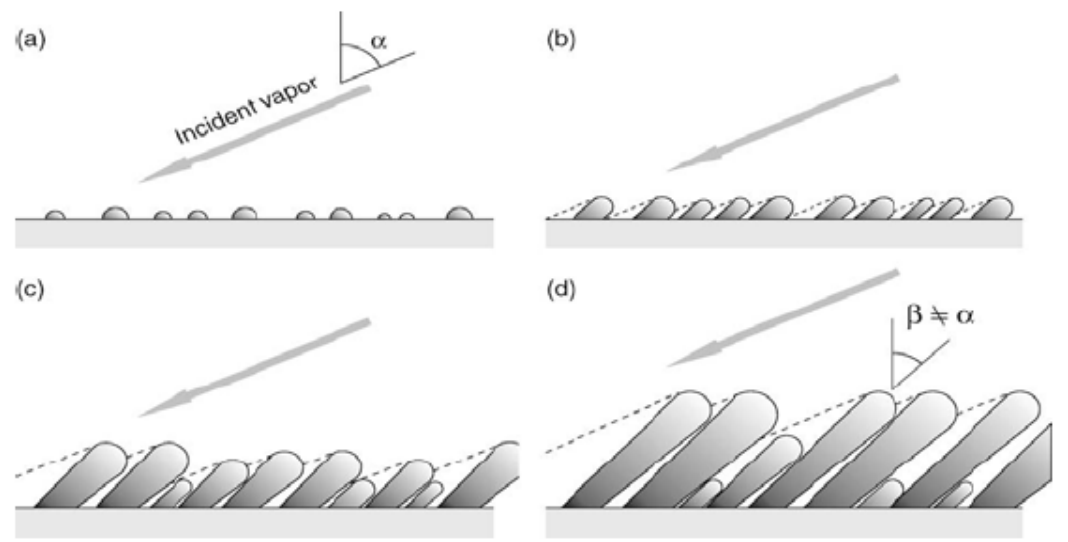

Figure 3.4GLAD Technique

As the deposition progresses, smaller nuclei and columns can become completely shadowed and stop growing. Figure 3.4(d) illustrates these phenomena. Eventually, only the top of nuclei are able to grow and develop into columns tilted towards the incident flux.

In Figure 3.4(d), the column tilt angle $\beta$ is 0 degree when a column is parallel with the substrate normal and would be 90 degree for parallel to the substrate surface. The tilt angle does not fully follow the incident angle $\alpha$. The growth of the column depends on several conditions, such as substrate temperature, deposition rate, pressure and substrate type, etc.

\subsubsection{Vapour-liquid-solid growth glancing angle deposition}

Vapour-liquid-solid(VLS) is a crystal growth technique that results in high surface area nanostructures that grow primarily in one dimension. ITO film can be grown with a selfcatalyzed VLS mode accessible at elevated substrate temperature. 
The VLS-GLAD is a technique that combines high substrate temperature and high deposition angles to produce unique morphologies. During the process, the GLAD method is used to control the vapour flux and the vapour-liquid-solid method is applied to induce ITO growth. Figure 3.5 demonstrates the VLS-GLAD parameter space relative to GLAD, VLS and planar deposition. The VLS-GLAD has a high deposition angle and elevated temperature. The VLS method is the most powerful silicon nanowire growth technique. But, one of major issue of this technique is the growth direction control. The VLS-GLAD technique can overcome this issue and grow silicon nanowire with a controlled geometry.

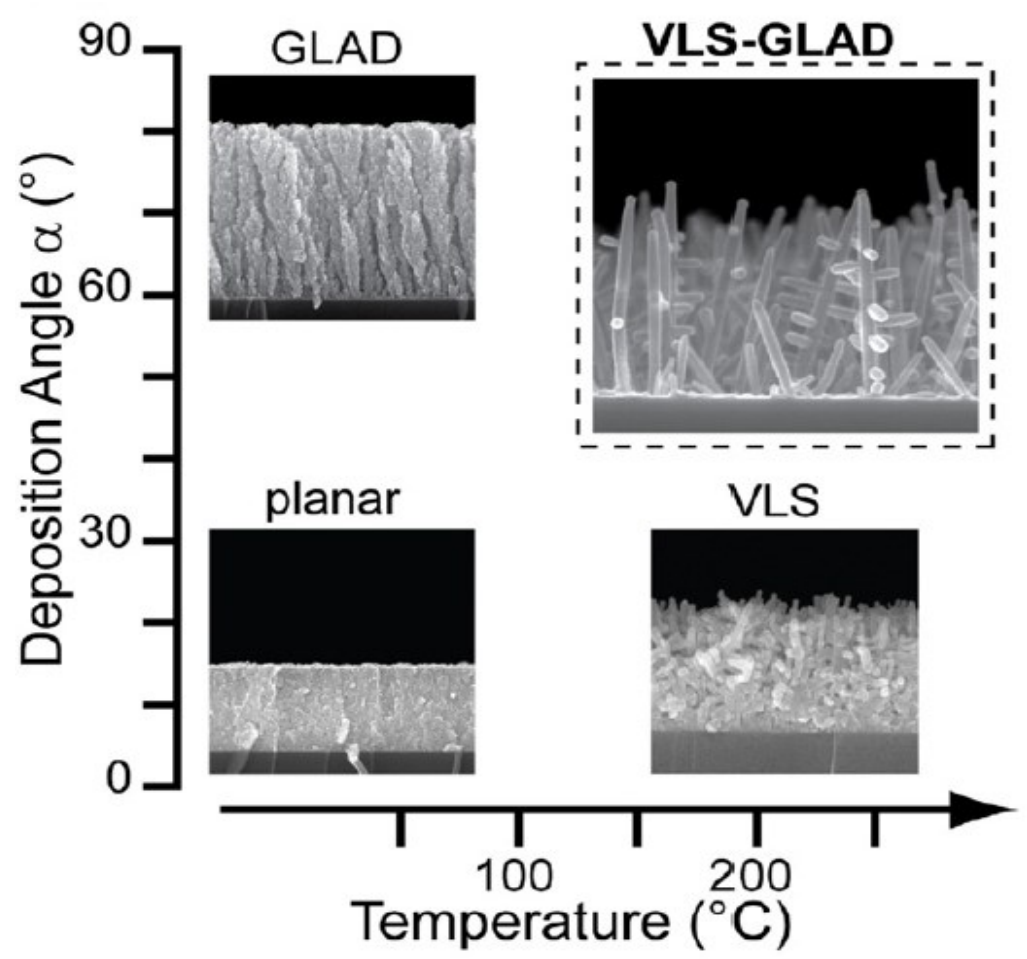

Figure 3.5Qualitative parameter space for ITO planar, glancing angle deposition,vapour-liquid-solid grown film and VLS-GLAD[31] 


\subsection{Amorphous Silicon Film Deposition}

\subsubsection{Overview of Low Pressure Chemical Vapor Deposition}

The amorphous silicon thin films were grown by Low Pressure Chemical Vapor Deposition (LPCVD). LPCVD is a thermal process that deposits film at low pressure. In this technique, a carrier gas, shown in Figure 3.6(a), is delivered into the reaction chamber (furnace tube) at a controlled temperature. As the gas passes over or come into contact with a heated substrate, as shown in Figure 3.6(b), it absorbs and then reacts to deposit a film. The temperature of the substrates is very critical and it is controlled by a feedback system using a thermocouple temperature sensor and electrical heating element, as shown in Figure 3.6(c).

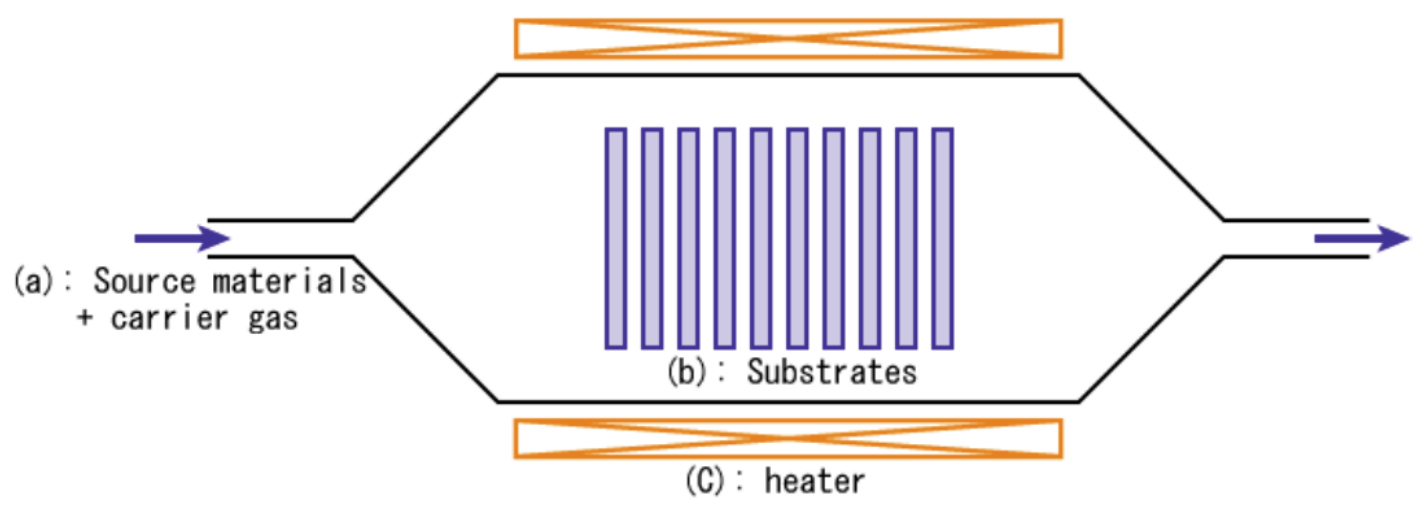

Figure 3.6LPCVD techniques [37]

At low pressure gas can diffuse more readily between the substrates giving a more uniform deposited film. 


\subsubsection{Growth Technique}

Amorphous silicon film is deposited using silane $\left(\mathrm{SiH}_{4}\right)$ as the source gas; the reaction is given in the following:

$$
\mathrm{SiH}_{4}(\mathrm{~g}) \rightarrow \mathrm{Si}(\mathrm{s})+\mathrm{H}_{2}(\mathrm{~g})
$$

The silane absorbs on the Si surface and decomposes to leave Si. At relatively high temperature the $\mathrm{SiH}_{4}$ molecule is mobile on the surface, giving a conformal layer. The temperature requirements to grow amorphous silicon can go as low as $75^{\circ} \mathrm{C}$. And, in order to avoid crystallization, the temperature should be lower than $600^{\circ} \mathrm{C}$. Here a temperature of $550^{\circ} \mathrm{C}$, a pressure of a few mTorr, and a flow rate in the sccm range were used. Under these conditions the growth rate was estimated to be $4 \mathrm{~nm} / \mathrm{min}$. Deposition times of $1 \mathrm{~min}$ and $2 \mathrm{~min}$ were used. No substrate cleaning was used prior to growth since nano-structured material is very fragile.

Figure 3.7 shown the amorphous silicon deposited ITO glass. For this example, the amorphous film is $8 \mathrm{~nm}$ in thickness. Compared with the pure ITO glass in Figure 3.2, the color of the sample has changed to brown.

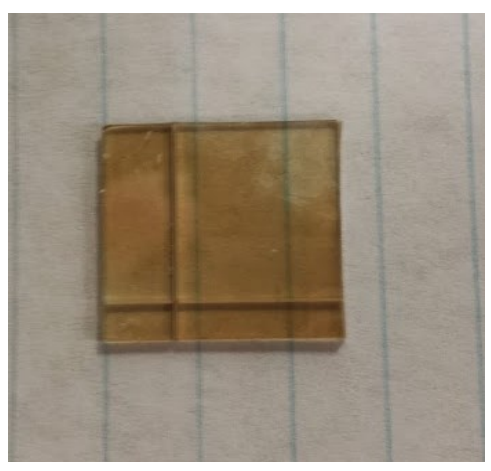

Figure 3.7ITO deposited with an amorphous Silicon film 


\subsection{Annealing}

Crystallization of amorphous silicon can be accomplished by several methods. Solid phase crystallization by furnace annealing at temperature in the range of 600 to $700^{\circ} \mathrm{C}$ is the simplest and the most popular technique. However, the disadvantage of this technique is the long heating duration. It will at least take 30 minutes at $600{ }^{\circ} \mathrm{C}$ for the crystallization to occur. Annealing under $600{ }^{\circ} \mathrm{C}$ is also possible but require much longer time. It is always trading between timing and temperature. Although heating the film to higher temperature will reduce the duration, there is a risk of substrate melting under high temperature.

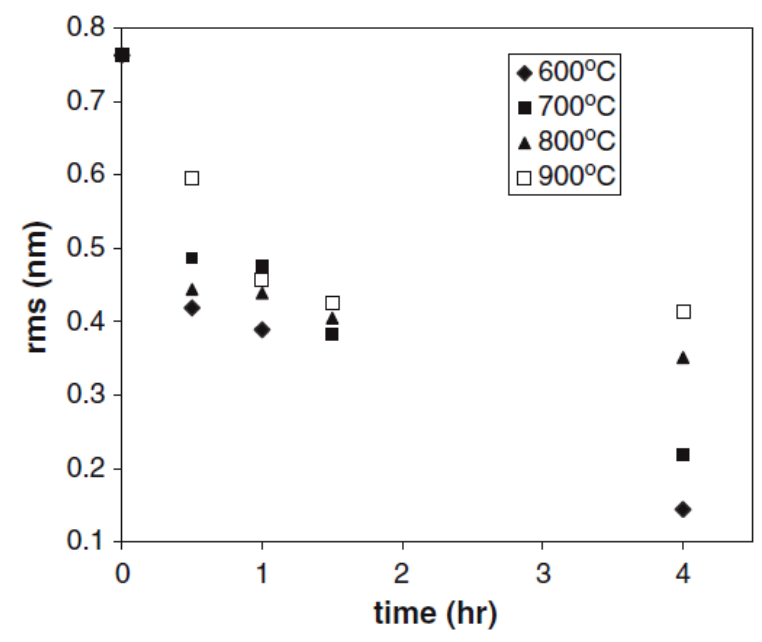

Figure 3.8Comparison of roughness of a-Si annealed at different temperatures

In M.Z. Lai 's research of thermal effects on LPCVD amorphous silicon[38]several annealing conditions were tested and measured using atomic force microscopy (AFM). Figure 3.8 shows the comparison of the roughness value obtained from AFM analysis. It is clear that as the annealing time increases from $0 \mathrm{~h}$ to $4 \mathrm{~h}$ the roughness at each temperature will decrease. Comparing the roughness at each temperature annealing at $600^{\circ} \mathrm{Cgives}$ the best results. 
Although annealing at $600{ }^{\circ} \mathrm{C}$ for $4 \mathrm{~h}$ gives the lowest roughness but the melting risk of the nanostructured ITO will increase as the time increases; the amorphous films were annealed at $600{ }^{\circ} \mathrm{C}$ for 60 minutes. This temperature is much less than the ITO melting point $\left(1730^{\circ} \mathrm{C}\right)$, thus the ITO won't collapse during the annealing. 


\subsection{SEM image of the fabricated film}

The fabricated $8 \mathrm{~nm}$ a-Si film on ITO substrate was send to University of Western Ontario for

SEM image. The thickness of the film is measured as $8 \mathrm{~nm}$ with a flat witness silicon substrate.

The result is shown in Fig. 3.9 and Fig. 3.10. From the top view of the film, it is clear that a-Si

was deposited on the ITO substrate. But from the cross section view in Fig 3.10, it is suggested

that, at least in some areas, thea-Si hasn't made a nice conformal coating of the ITO trees, but

instead has filled in the top branches of the trees, shadowing the structure underneath from

further deposition .

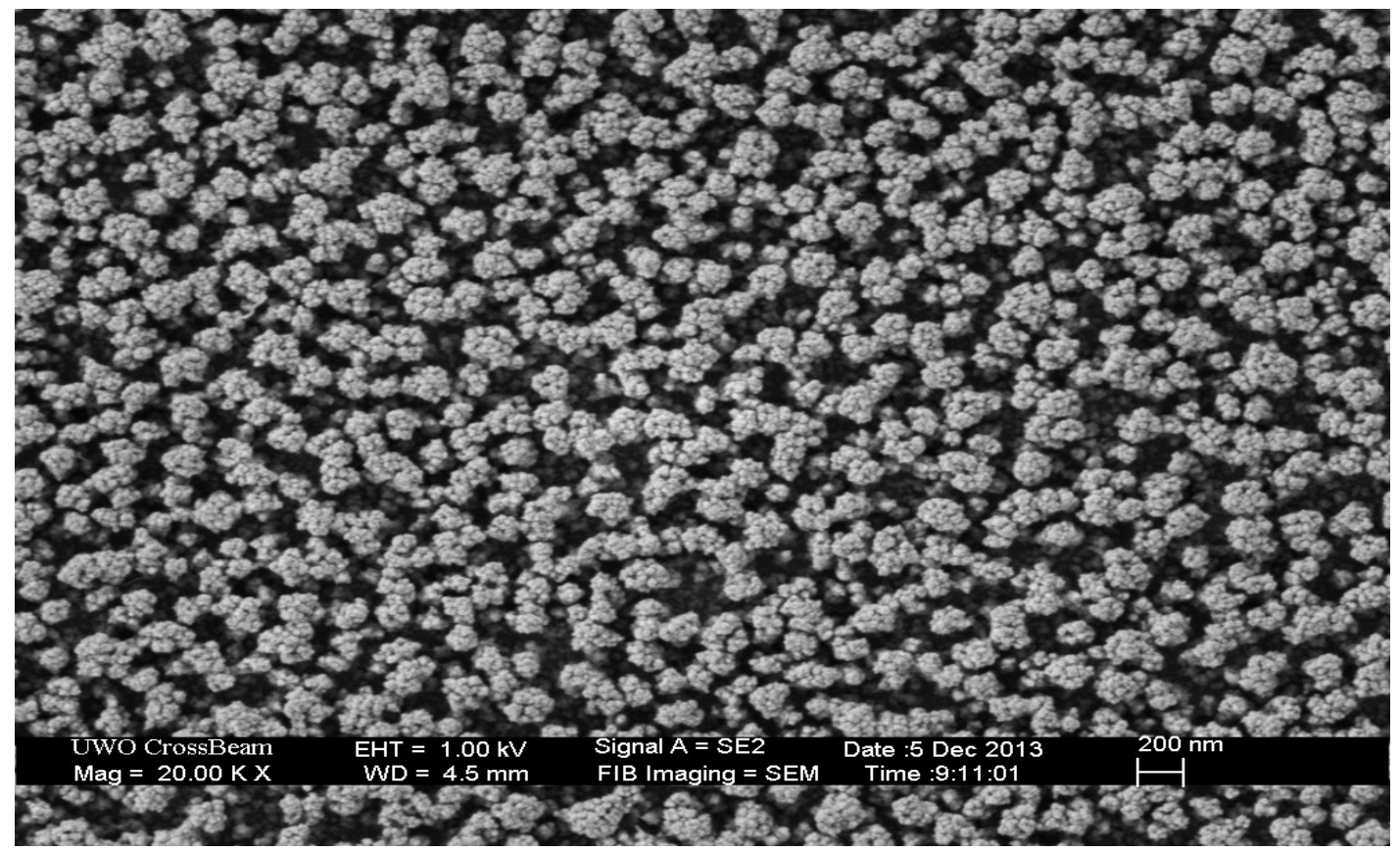

Figure $3.98 \mathrm{~nm}$ a-Si film top view 


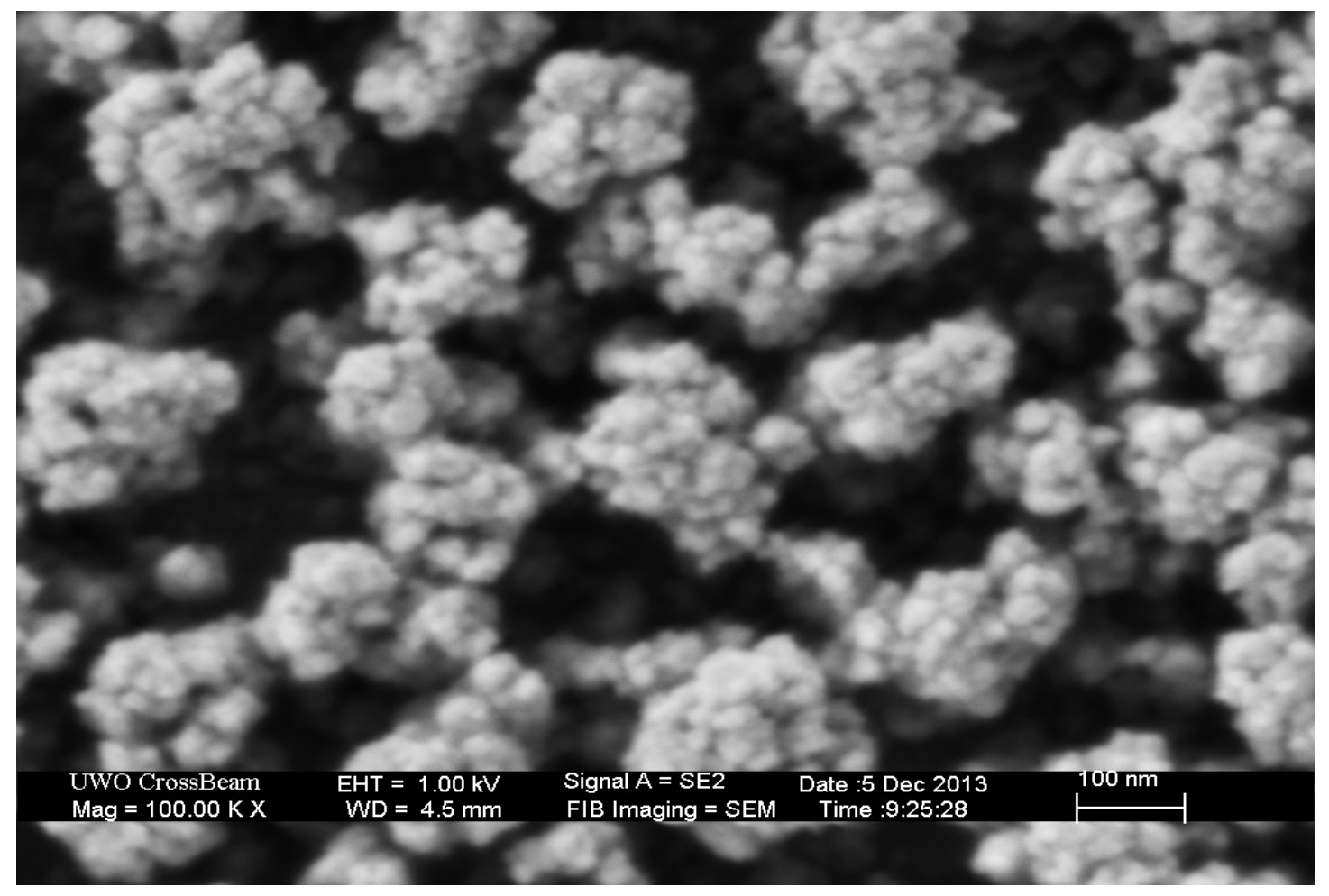

Figure $3.108 \mathrm{~nm}$ a-Si film top view

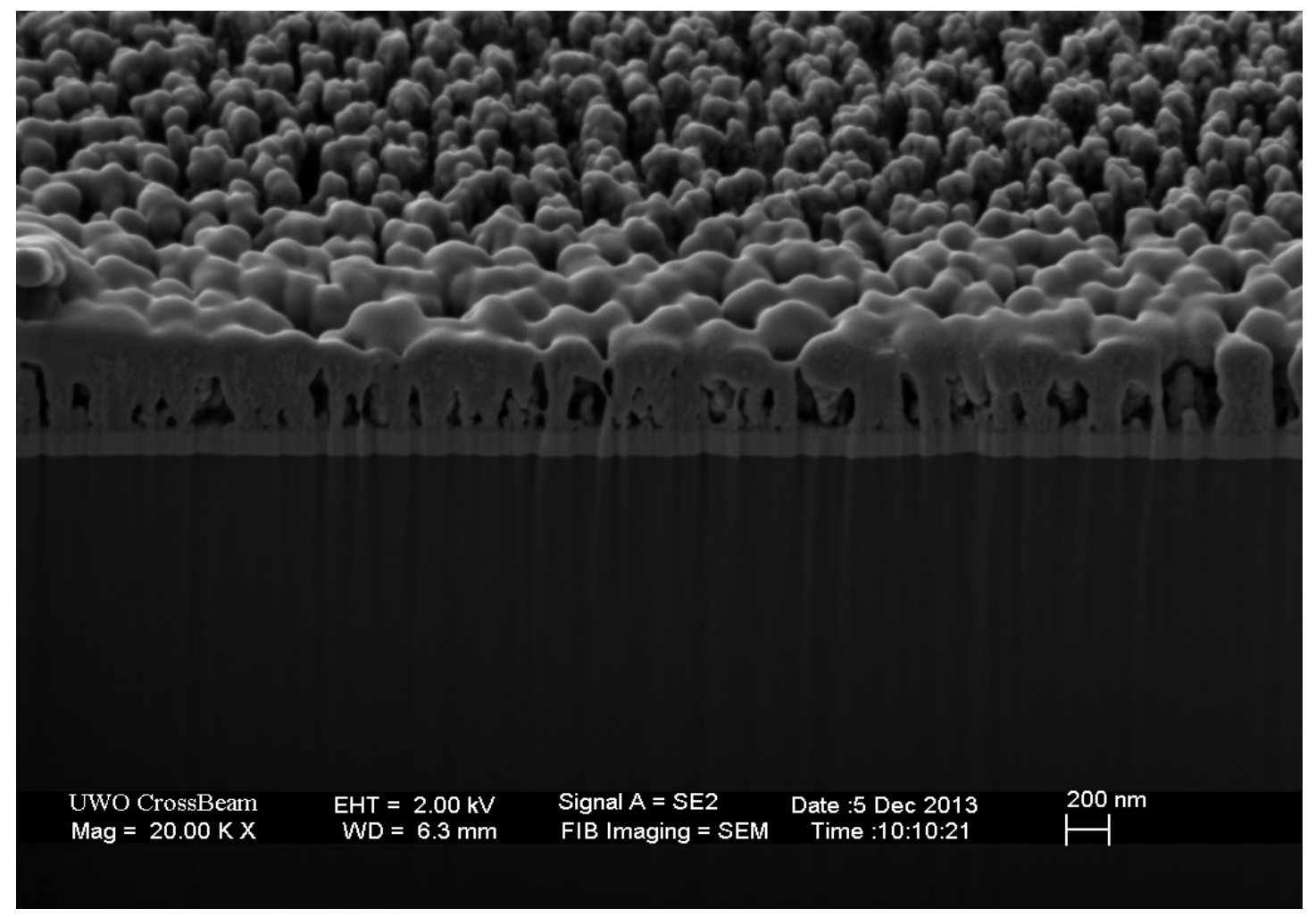

Figure $3.118 \mathrm{~nm}$ a-Si film cross section view 


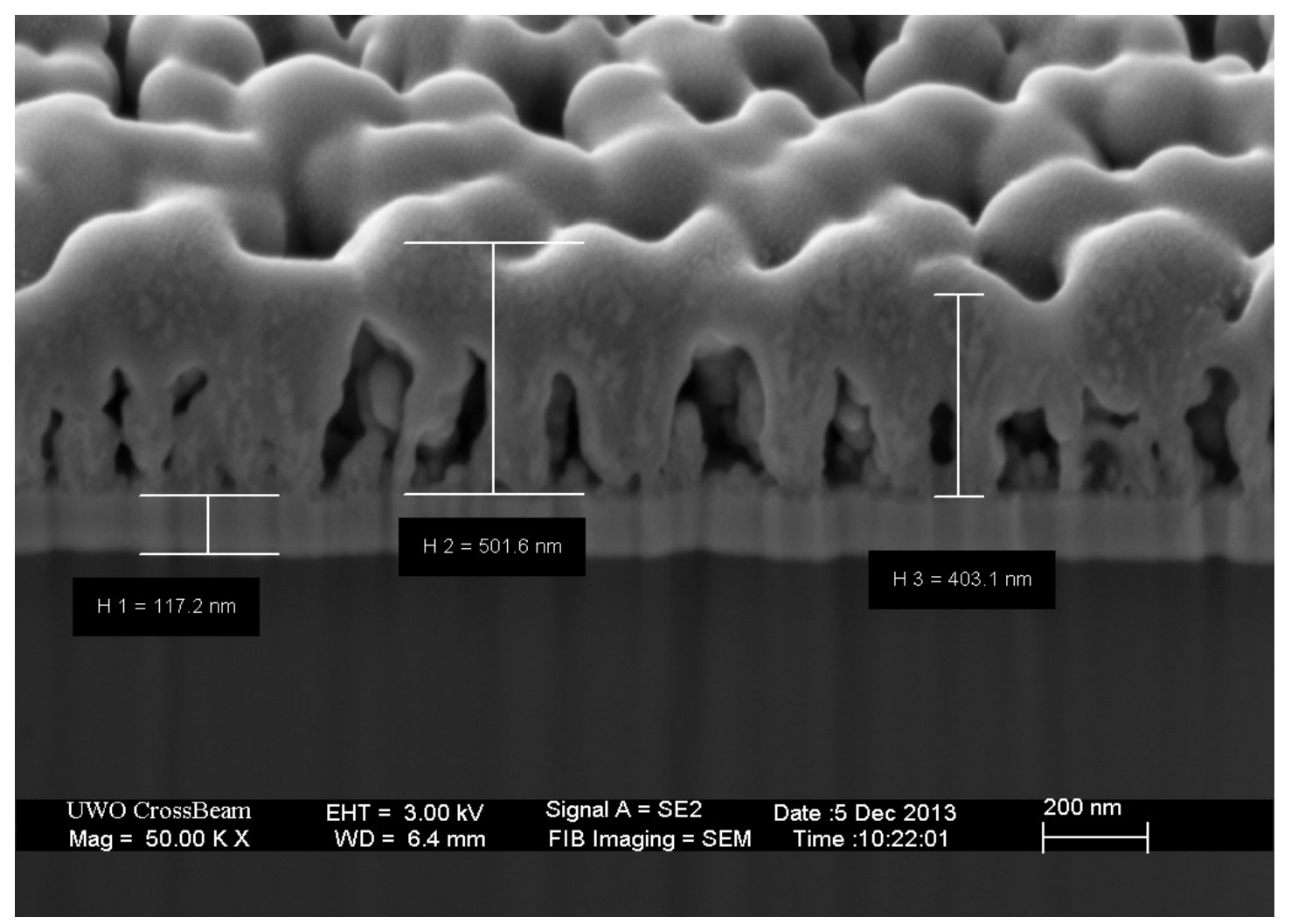

Figure $3.128 \mathrm{~nm}$ a-Si film cross section view

\subsection{Conclusion}

The substrate of this new film was a column like ITO film fabricated with vapour-liquid-solid growth glancing angle deposition at the University of Alberta. The top layer of this film was deposited with a 4-8 nm thick a-Si film and annealed for crystallization. The SEM image of this film shows that deposition of silicon film did not make a nice conformal coating. This will result in a poor bandgap modification. 


\section{Chapter IVExperiment: Optical Characterization of}

\section{Nanostructured Silicon Film}

\subsection{Visible-UV and IR Spectroscopy Experiment}

\subsubsection{Overview}

The optical property of thin film can be characterized by ellipsometry, absorption and reflectance spectroscopy. The ellipsometry uses the fact that the polarization of the light changed when it is reflected from the surface of the material. It measures the phase change and amplitude change of the s-direction and p-direction. However, it is difficult to measure multi-layer film with ellipsometry. We will use both absorption and reflectance spectroscopy to measure the bandgap of the nanostructured film.

In order to measure the reflectance and transmittance versus wavelength, a spectrometer station is needed. There are two types of configurations for spectrometers. Both of them need a light source, a monochromator and a photo detector. The first configuration as shown in Figure 4.1(a).Light from the source is sent to the sample, and then the reflected or transmitted light is wavelength dispersed by a monochromator and recorded by a photo detector. The second configuration is shown in Fig. 4.1(b). Instead of sending the light to the sample, the light from the source is first dispersed by a monochromator, and the resulting monochromatic light is sent to the sample and recorded by a photo detector. 


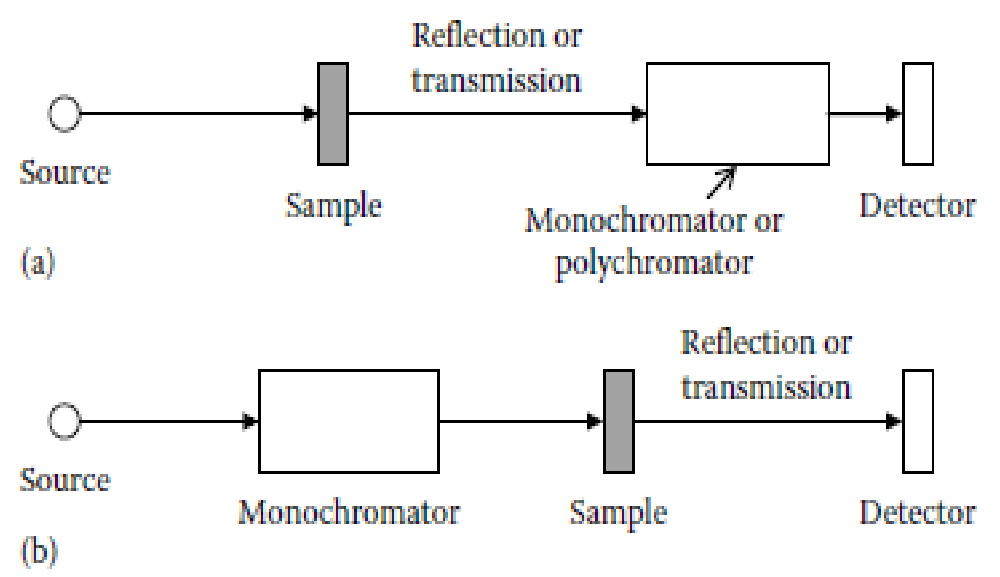

Figure 4.1Spectrometer configuration [39]

\subsubsection{Experimental Tools}

\subsubsection{Light sources}

The light source is the most important component in any optical experiment. A broadband light is needed to cover the required spectral range. There are various types of lamp that can produce stabilized wide range light. Quartz Tungsten Halogen (QTH) lamps are popular visible and near infrared sources because of their smooth spectral curve and stable output. They do not have the sharp spectral peaks that arc lamps exhibit, and they emit little UV radiation. Also, a halogen lamp can be operated at a higher temperature than a standard gas-filled lamp of similar power and operating life, producing light of a higher luminous efficacy and color temperature[39].

\subsubsection{Monochromator}

The main purpose of a monochromator is to disperse light into its component spectral wavelengths. More specifically, it will separate a light colors and isolate one wavelength for measurements. 


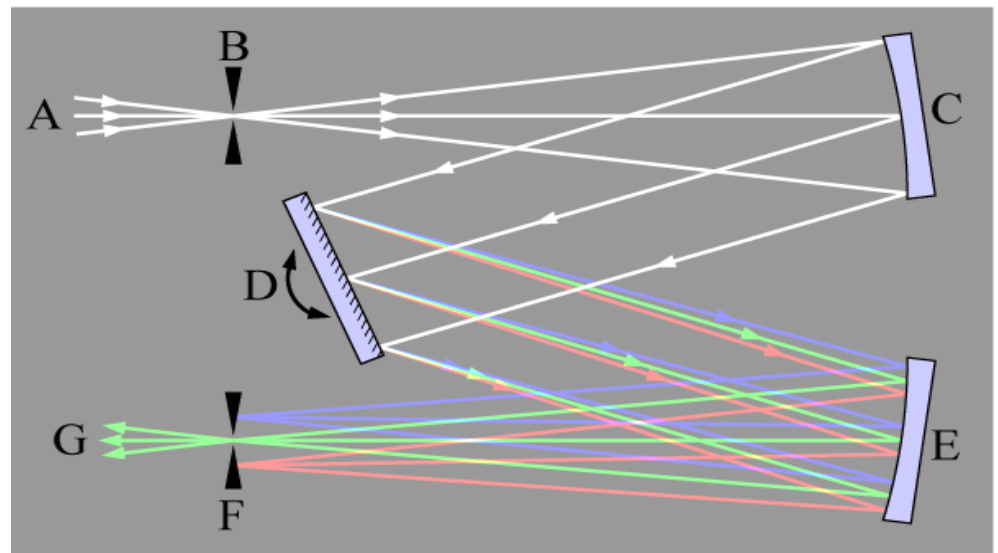

Figure 4.2Monochromator theory [41]

Fig. 4.2 illustrates how a monochromator works. At point A, a light is focused onto an slit B and then collimated by a mirror $\mathrm{C}$. The collimated beam is diffracted from a rotatable grating $\mathrm{D}$. The dispersed beam focused by mirror E at the slit F. Each wavelength of light is focused to different positions at the slit, and the wavelengthes that will be transmitted through the slit are controlled by the rotation angle of the grating $\mathrm{D}$.

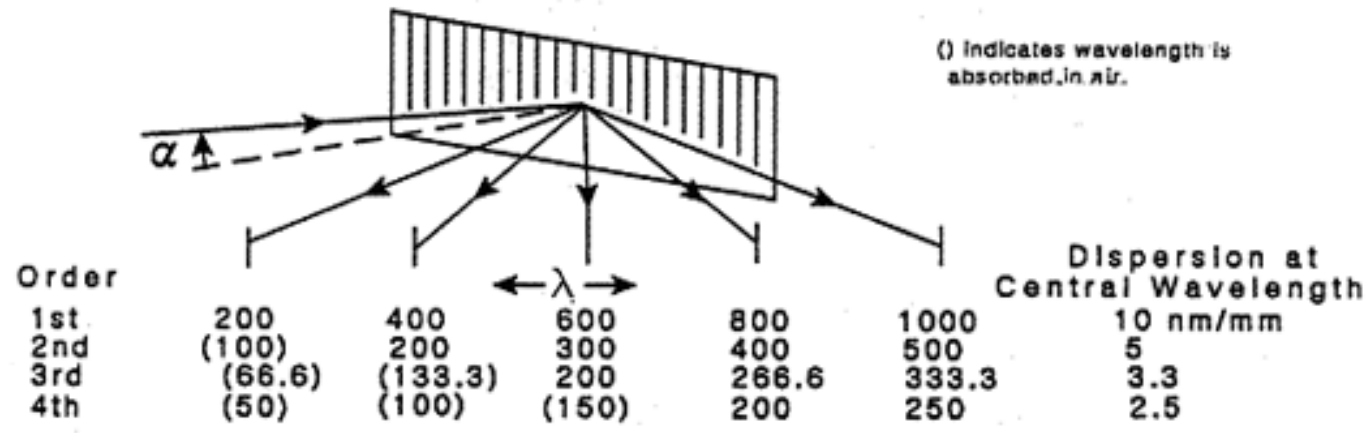

Figure 4.3Dispersion and order[42]

There is a higher order wavelength contamination problem in obtaining a monochromatic light with a grating. Fig. 4.3 illustratesa first order spectrum from $200 \mathrm{~nm}$ to $1000 \mathrm{~nm}$. The figure shows that the first order of a longer wavelength may diffract at the same angle as the second order of a shorter wavelength. 
For instance, a light source emits a continuum of wavelengths from $200 \mathrm{~nm}$ to $1000 \mathrm{~nm}$ then at the physical location of $800 \mathrm{~nm}$ in first order (Figure 4.3) wavelengths of 400, 266.6, and $200 \mathrm{~nm}$ will also be present and available to the same detector. In order to detect the desired light at 800 $\mathrm{nm}$, filters must be used to eliminate the higher orders.

\subsubsection{Detector}

Common detectors used in optical experimental are silicon and germanium photodiodes. Silicon photodiodes have a wavelength range of roughly 400 to $1100 \mathrm{~nm}$ while germanium photodiodes work from about $700 \mathrm{~nm}$ to $1800 \mathrm{~nm}$. However, we can get a $400 \mathrm{~nm}$ to $1800 \mathrm{~nm}$ range by combining the data from Si photodiode with the data from a Ge photodiode.

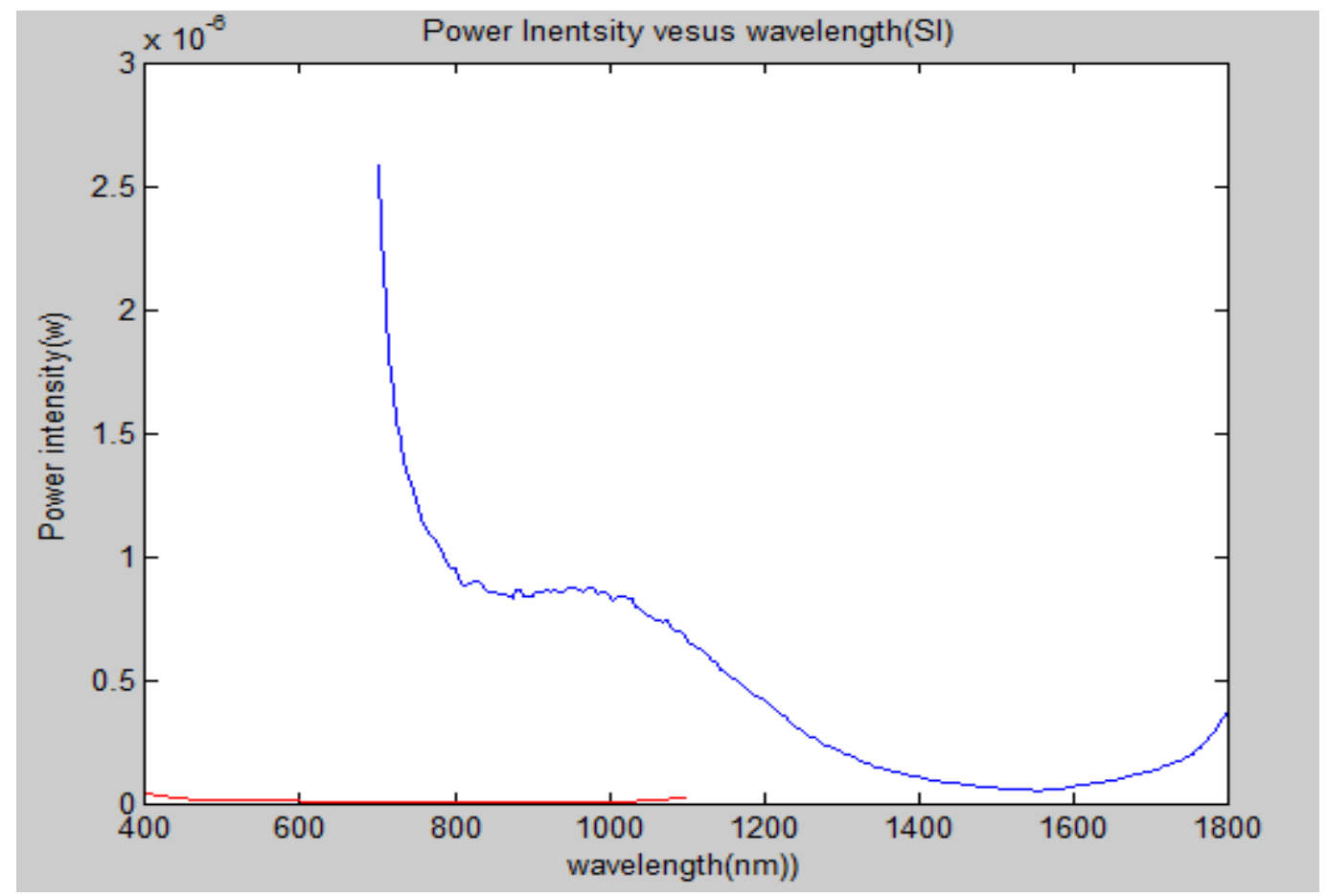

Figure 4.4Photodiode sensitivity 
Although the germanium photodiode works over 700 to $1800 \mathrm{~nm}$, it does not have a reliable performance for wavelengths shorter than $1100 \mathrm{~nm}$.Fig. 4.4 indicates the power intensity recorded by each photodiodes when the light source is off. The ideal power intensity should be zero for both Si and Ge photodiodes since there is no light hitting the detector. The power intensity of the Si photodiode is the red curve and the intensity is indeed close to zero. But, the intensity in the Ge photodiode or the blue curve decreased dramatically in the 700 to $1100 \mathrm{~nm}$ range. The Ge detector does not provide reliable results. Therefore, we will avoid using Germanium photodiode from 700 to $1100 \mathrm{~nm}$ range.

\subsubsection{Visual Engineering Environment}

Agilent VEE is a graphical dataflow approach to automated test, measurement, and data analysis. It is a powerful tool to integrate all the optical devices and to enhance the experiment efficiency. Also, it is really helpful in collecting and processing optical and electrical data.

The following absorption experiment and reflection experiment were conducted by using VEE programs. These programs control the hardware tools such as the monochromator, second order filter and optical detectors.

\subsection{Absorption Experiment}

The configuration for measuring transmitted power and the incident power is shown as Figure 4.5. The light from the source travels into a monochromator and wavelengths are spatially separated as a function of wavelength. The separated light first will pass through a second order 
filter to eliminate higher order modes and then travels through a lens. The lens will concentrate the light to avoid power losses. Then, concentrated light will pass through the sample holder. An initial measurement is taken with the sample holder empty in order to measure the incident power. The sample is then inserted and the transmitted power measured. After the light passes through the sample, the light is concentrated again and then passes into a beam splitter. The beam splitter allowed us to record the transmitted power by both Si and Ge detectors at the same time. Thus, a wider wavelength range is available.

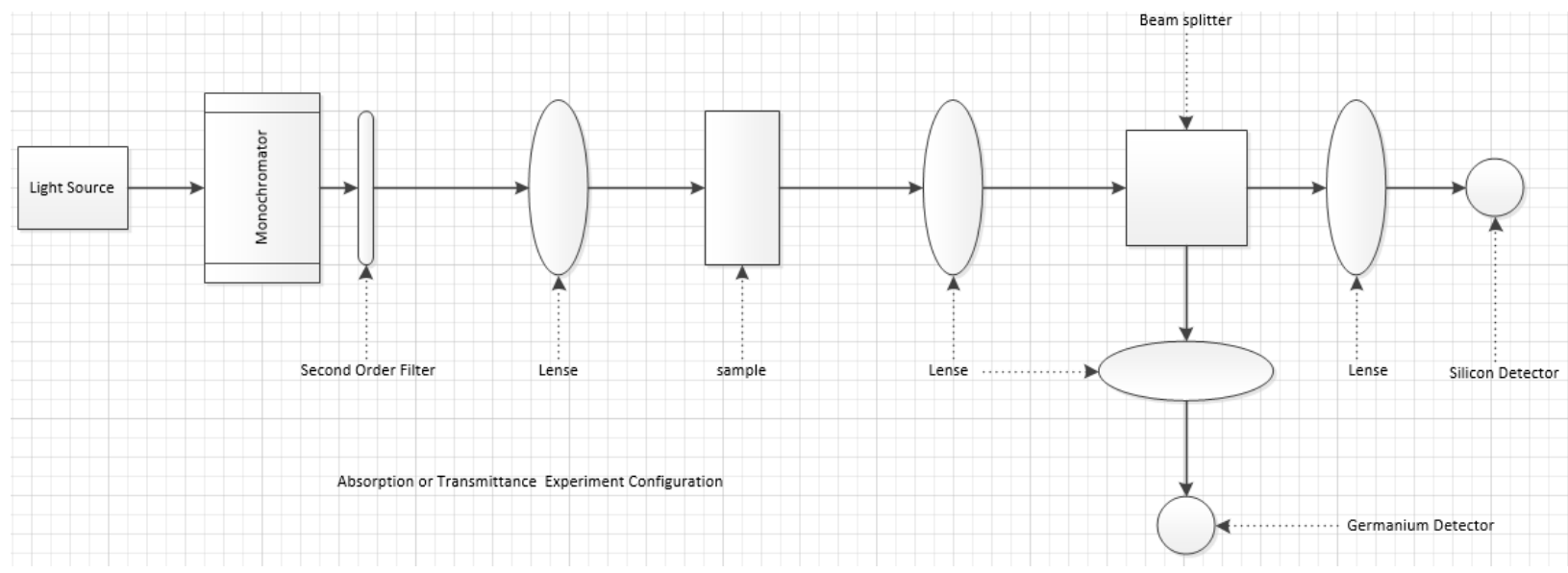

Figure 4.5Absorption configuration

The absorbed power is obtained by subtracting the transmitted power and reflected power from the incident power.

Absorption $=$ IncidentPower - Transmittedpower - Reflectedpower 


\subsection{Reflection Experiment}

\subsubsection{Overview}

There are two kind of optical reflection configuration. The first one is called the near normal incidence, which means the incident light has very small incident angle. As shown in Fig. 4.6(a), the intensity of the reflected light $\mathrm{I}(\omega)$ and the incident light $\mathrm{I}_{0}(\omega)$ are measured to obtain the reflectance $R=\frac{I(\omega)}{I_{0}(\omega)}$. A more accurate approach is to use a reference material with known reflectance $R_{r}(\omega)$. The intensity of this reference material is measured $\operatorname{asI}_{r}(\omega)$. As a result, the reflectance $\mathrm{R}$ can be written asR $(\omega)=\left[\frac{\mathrm{I}(\omega)}{\mathrm{I}_{\mathrm{r}}(\omega)}\right] \mathrm{R}_{\mathrm{r}}(\omega)$.

(a)

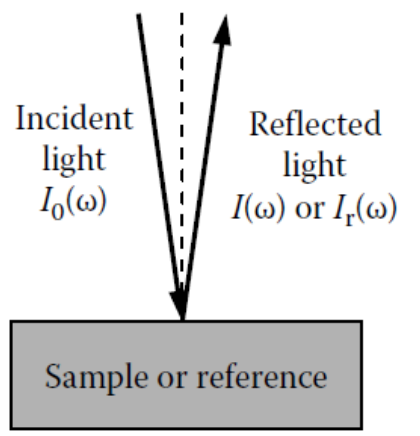

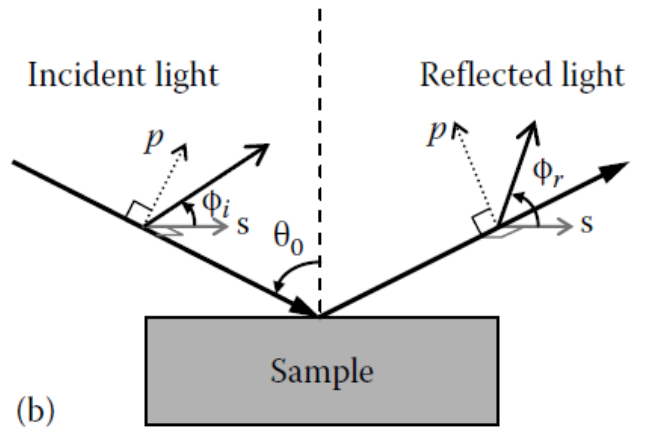

(b)

Figure 4.6Optical reflection configurations [43]

The second method, shown in Figure 4.6(b), uses grazing angle incidence. In this method, the thick open arrows indicate the polarization vectors, and $\mathrm{p}$ and $\mathrm{s}$ denote their components parallel and perpendicular to the plane of incidence, respectively. $\theta_{0}$ is the angle of incidence and $\varphi_{\mathrm{i}}$ and $\varphi_{\mathrm{r}}$ are the polarization direction. Grazing angle incidence is used with ellipsometry technique for deriving the reflection. 


\subsubsection{Experimental Configuration}

The experimental configuration used here is shown in figure 4.7. The beam produced by the light source is separated by the monochromator and then filtered by the second order filter to eliminate higher orders. Then, the beam will be focused at center of the sample film. In order to measure the reflected power, the incident angle is set to be 30 degrees. The reflected light will be focused at the center of the photo detector.

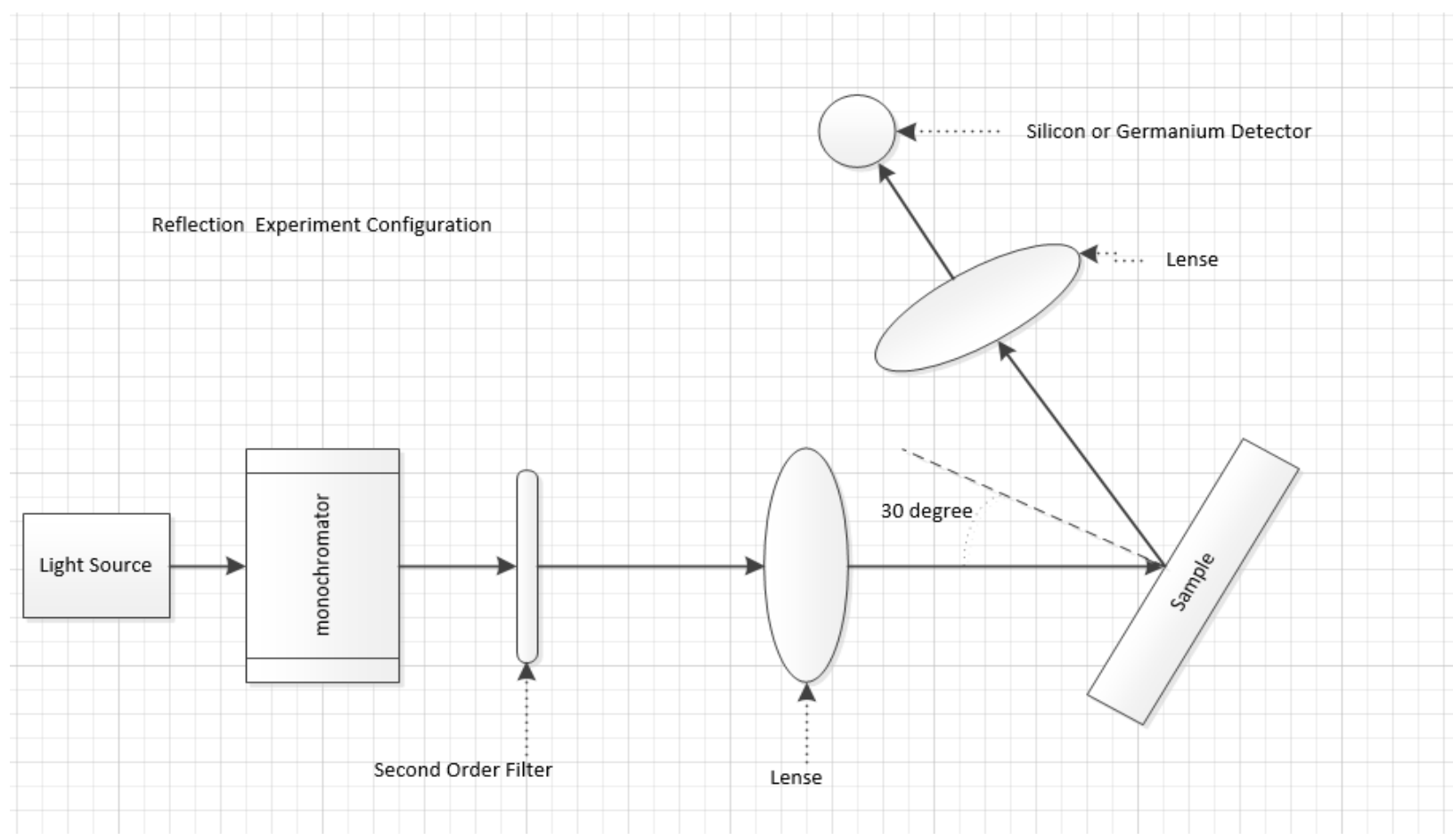

Figure 4.7Reflection configuration

As mentioned before, a reference material can be used to increase the accuracy. Optical mirrors such as Al, Ag and Au are the common choice as reference material. 


\subsection{Example of Silicon Wafer Characterization}

\subsubsection{Silicon Wafer example}

In order to test the experimental configuration, a piece of silicon wafer was measured as a control sample. Silicon has a bandgap energy at $1.11 \mathrm{eV}$ which corresponds to $1116.9 \mathrm{~nm}$ wavelength. Therefore, we expect to see a sharp change in absorption coefficient at around $1116.9 \mathrm{~nm}$. The reflectance should also decrease as the photon energy decreases. Typical silicon has a reflectance around $50 \%$ to $30 \%$ between 400 to $1000 \mathrm{~nm}$ at normal incident angle.

\subsubsection{Absorption Results}

The extracted absorption coefficient for the silicon sample is shown in Fig.4.8. The absorption between 400 to $1100 \mathrm{~nm}$ is 1 or $100 \%$, which means no detectable light passes through the silicon wafer. At $1100 \mathrm{~nm}$, the absorption drops dramatically to 0.65 . We can conclude that photons having energy less than $1.12 \mathrm{ev}$ cannot be absorbed by electrons in silicon. The apparent absorption of the silicon wafer is still very high above $1100 \mathrm{~nm}$. That is because the back surface of the silicon wafer is rough and the light gets scattered. 


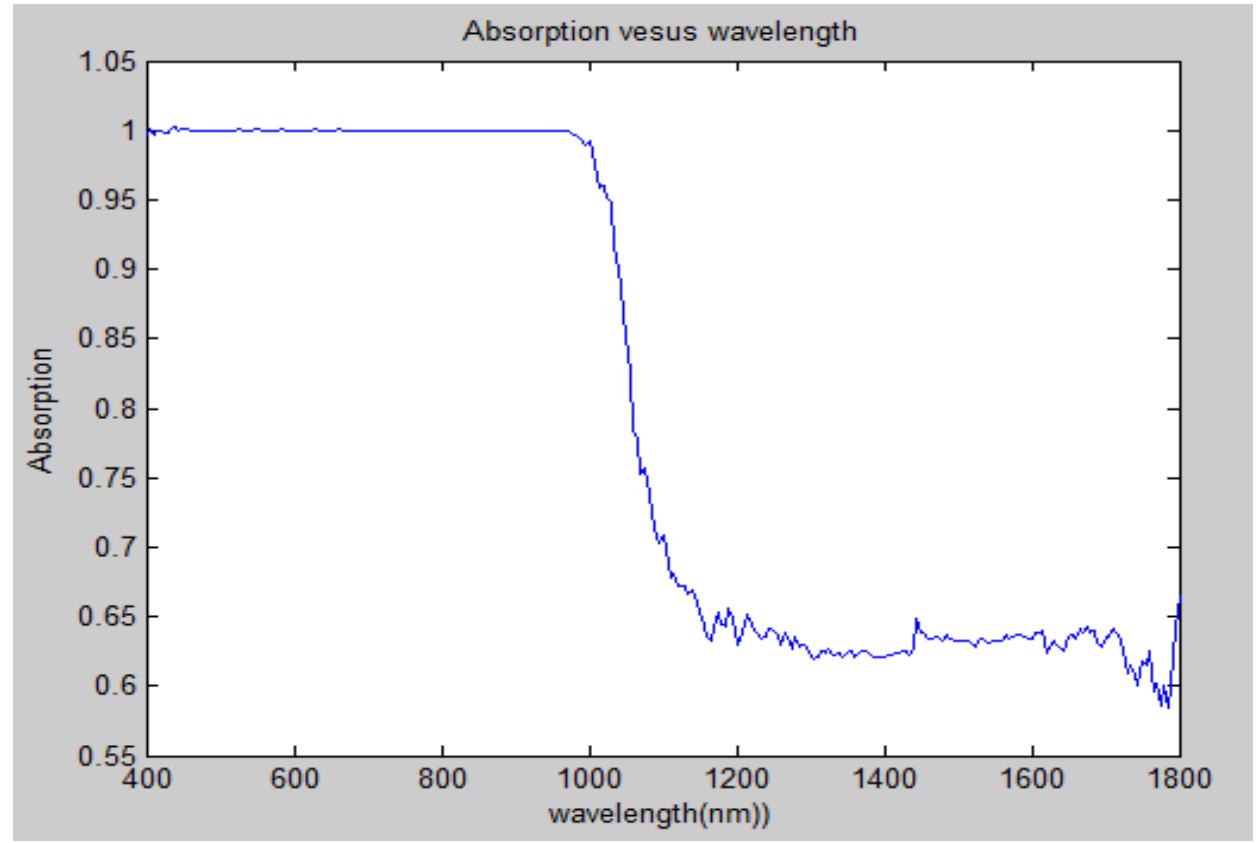

Figure 4.8Silicon wafer absorption

\subsubsection{Reflection Results}

The reflection results are closer to expectation than the absorption results. As shown in Figure 4.9 , the reflectance decreased from 0.4 at $400 \mathrm{~nm}$ to 0.2 at $1800 \mathrm{~nm}$. The results are still lower than the value in Chapter 2. This is caused by the roughness of the silicon wafer. Since the silicon wafer is not flat, part of the incident light will scatter outside the detector. A possible enhancement for this is using an integrating sphere detector to collect all the light. 

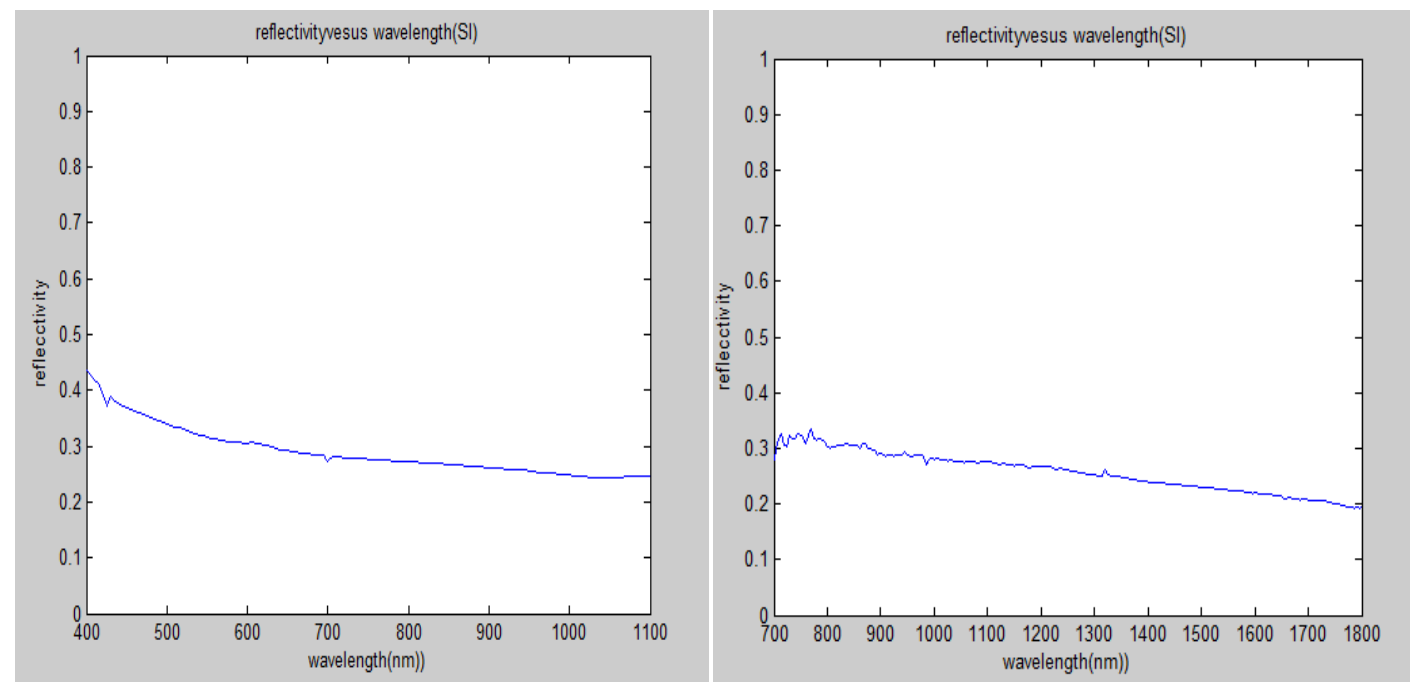

Figure 4.9Silicon wafer reflections

\subsection{Conclusion}

The bandgap of the new film was determined from the absorption and reflection data. That data was measured from the spectrometer including light source, monochromator and photo detector. Etc. The absorption experiments pass the light through the film and collecting the transmitted light to calculate the absorption. The reflection experiment collects the reflected light with a 30 degree incident light. The set up was tested with a single polished silicon wafer and give a reasonable result. 


\section{Chapter V Results and Discussion}

\subsection{Overview of the Samples}

Five different films were deposited in these experiments, with properties summarized in Table 5.1. The first film consisted of $8 \mathrm{~nm}$ of aSi deposited on a glass substrate. The second film used an identical silicon layer but with an ITO-coated glass substrate. The third and fourth films were deposited on Nano-structured ITO glass prepared by the University of Alberta, with Si film thickness of 8 and $4 \mathrm{~nm}$. The third sample was eventually annealed to crystallize the silicon film, which gives us the fifth film.

\begin{tabular}{|l|c|c|c|}
\hline & Substrate & Silicon & Film Thickness \\
\hline Film 1 & Glass & Amorphous Silicon & $8 \mathrm{~nm}$ \\
\hline Film 2 & ITO Glass & Amorphous Silicon & $8 \mathrm{~nm}$ \\
\hline Film 3 & Nano-structured ITO & Amorphous Silicon & $8 \mathrm{~nm}$ \\
\hline Film 4 & Nano-structured ITO & Amorphous Silicon & $4 \mathrm{~nm}$ \\
\hline Film 5 & Nano-structured ITO & Crystallized Silicon & $8 \mathrm{~nm}$ \\
\hline
\end{tabular}

Table 5.1: Summary of samples fabricated

Two different methods were applied to analyze the bandgap of these samples. The first, involved the direct measurement of absorption, which provided a rough estimate of the bandgap. The second method involved extraction of the complex refractive index from measured reflectance data using the Kramers-Kronig equation. Using this second approach, the absorption coefficient for the last three films was calculated. These two methods will be discussed separately in the following sections. 


\subsection{Absorption Analysis}

The absorption analysis studies the percentage of light absorbed by the film relatively to the incident light by measuring the transmitted light. In this analysis, the reflection is assumed to be zero at normal incidence. Thus, the absorption can be obtained by:

$$
\text { Absorption }=1-\text { Transmission; }
$$

The absorption at short wavelength $(400 \sim 1000 \mathrm{~nm})$ is mainly due to interband absorption. As a result, the bandgap of a semiconductor material can be determined by analyzing its absorption edge.

In this section, the absorption of several films was measured and compared. First, in Section 5.2.1, the8nm thick amorphous silicon deposited on glass, regular ITO glass and nano-structured ITO glass were measured. Then, in order to analyze how the thickness of deposited amorphous silicon alters the absorption; a 4nm thick amorphous silicon film was measured and compared to the $8 \mathrm{~nm}$ thick a-Si film. In the end, to study the influence of crystallization on absorption, the $8 \mathrm{~nm}$ thick crystallized silicon was measured.

\subsubsection{Amorphous silicon deposited on Glass, ITO Glass and Nano-structured ITO}

Three kinds of substrate were deposited with $8 \mathrm{~nm}$ thick amorphous silicon films and characterized by their absorption. Amorphous silicon typically has a bandgap in the 700 to 850 $\mathrm{nm}$ range[44]. With different substrates, the absorption performance may be altered. 


\subsubsection{Absorption Of Amorphous Thin Film}

The absorption edge of the 8nm thick amorphous silicon film, deposited on a glass substrate was measured. The substrate is 7059 glass and has a transmittance great than $90 \%$ in the range 380 $2200 \mathrm{~nm}$. In figure 5.1, the red curve and black curve corresponds to the absorption of bare 7059 glass and the absorption of the glass with $8 \mathrm{~nm}$ amorphous silicon film coating, respectively.

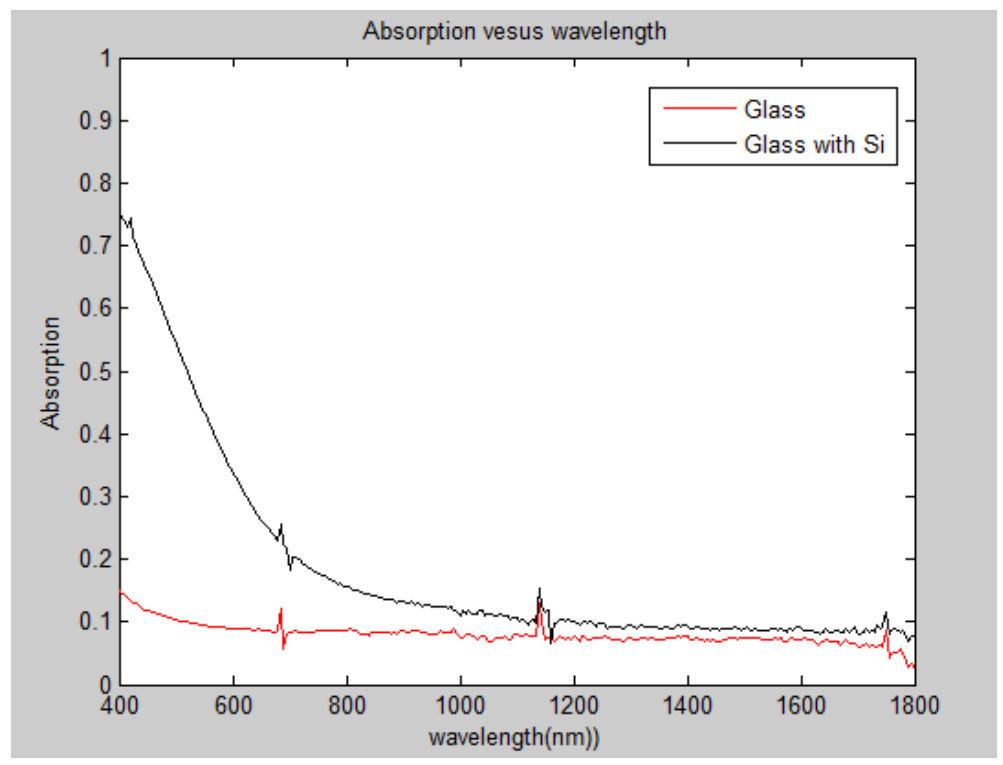

Figure 5.1Glass with and without a-Si coating

As expected, the glass (red curve) has $10 \%$ absorption or $90 \%$ transmittance from 400 to 1800 $\mathrm{nm}$. However, after the silicon deposition, the absorption increased over the 400 to $1000 \mathrm{~nm}$ range. For the amorphous silicon deposited sample (black curve), the absorption is $75 \%$ at the $400 \mathrm{~nm}$ and decreases from $75 \%$ to $20 \%$ over the 400 to $800 \mathrm{~nm}$ range. The absorption begins to bottom after the wavelength reaches $800 \mathrm{~nm}$. Therefore, we can conclude that the band gap for the $8 \mathrm{~nm}$ amorphous silicon film is found between $750 \mathrm{~nm}$ and $850 \mathrm{~nm}$. 


\subsubsection{Absorption Of a-Si Thin Film On ITO Glass}

An $8 \mathrm{~nm}$ thick amorphous silicon film was also deposited on a regular ITO coated glass substrate. The absorption of ITO glass and ITO glass with a-Si are shown in Figure 5.2. The red curve or the ITO absorption is between $30 \%$ to $15 \%$ from 400 to $1000 \mathrm{~nm}$ and increases up to $80 \%$ from 1000to $1800 \mathrm{~nm}$. ITO has a bandgap around $4 \mathrm{eV}$ and thus has a high transmittance from 400 to $1000 \mathrm{~nm}$. However, due to free electron absorption, the absorption increases for wavelengths greater than $1000 \mathrm{~nm}$.

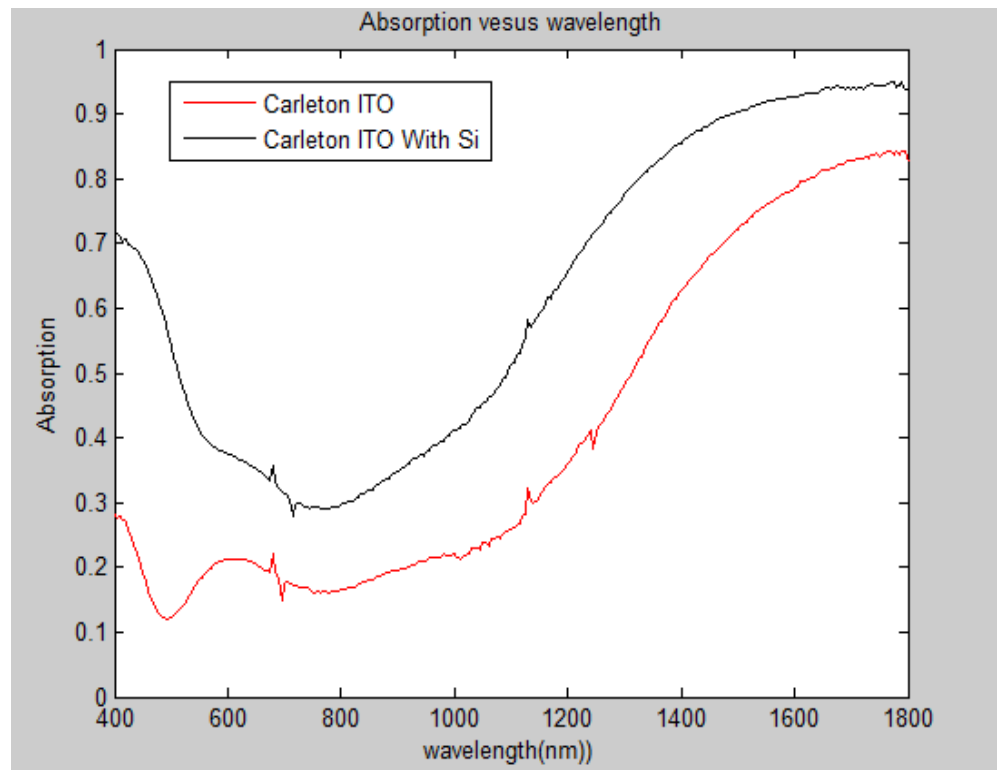

Figure 5.2ITO $v s$ a-Si on ITO

The a-Si deposited ITO(black curve) has a higher absorption than ITO glass over all wavelengths, but the difference of increase in absorption from $800 \mathrm{~nm}$ to $1800 \mathrm{~nm}$ is approximately constant. This is possibly because the ITO substrates have different properties. 


\subsubsection{Absorption of a-Si Thin Film on Nano-structured ITO}

The absorption of nano-structured ITO (black curve) is shown in Figure 5.3. It has similar absorption over 400 to $1000 \mathrm{~nm}$ range compared to regular ITO in Figure 5.2. But, the free electron absorption is lower in the nano-structured ITO. This is possibly because the nano structured ITO may have a lower free carrier concentration than regular ITO.

Comparing the a-Si coated nano-structured ITO (red curve) with the uncoated nano-structured ITO (black curve), the former has a higher absorption in the range of 400 to $800 \mathrm{~nm}$. This is because the amorphous silicon absorbs photons with higher energy than its band gap. However, in the range of 1000 to $1800 \mathrm{~nm}$, the dominant free carrier absorption of the nano-structured ITO entered and resulted a $10 \%$ to $40 \%$ absorption enhancement. But, in a-Si deposited nanostructured ITO, the free electron was trapped under the a-Si film and therefore the free electron absorption does not have evident influence. The absorption tends to stays around $20 \%$ from 1000 to $1800 \mathrm{~nm}$.

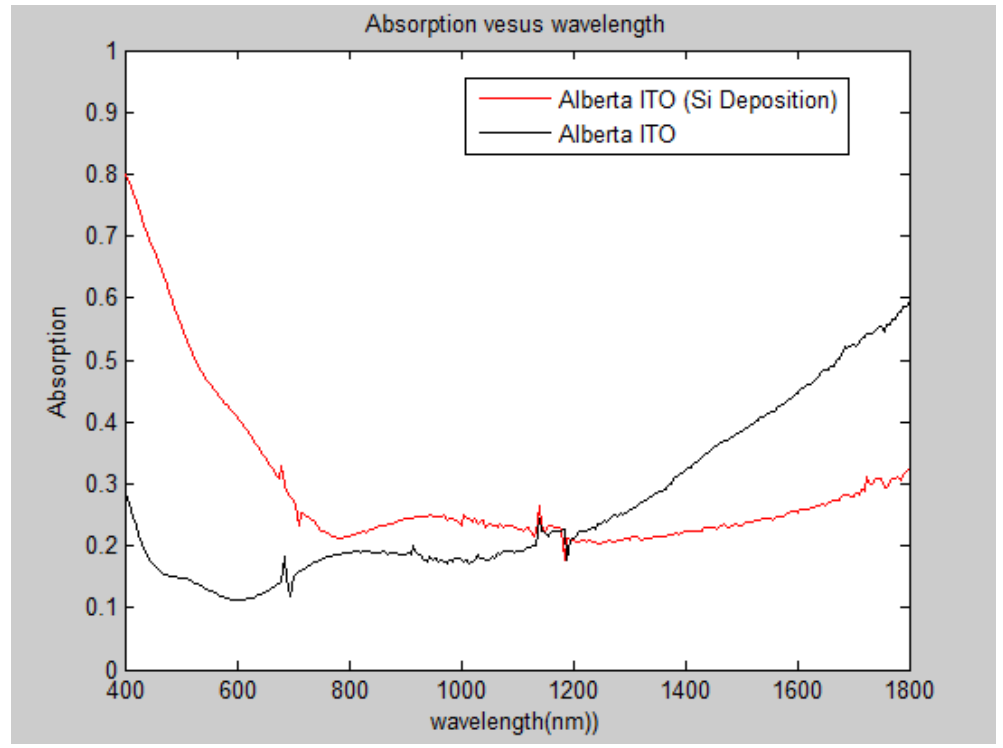

Figure 5. 30ptical absorption of nano-structured ITO vs a-Si on nano-structured ITO 


\subsubsection{Absorption of a-Si on different substrates}

The previous sections introduced three kinds of substrate for a-Si deposition and their absorption results. Each of them has a unique absorption curve as shown in Figure5.4. In the following figure, over the range 400 to $800 \mathrm{~nm}$ band to band absorption in the a-Si dominates and the three curves have a similar shape. But, the nano-structured ITO substrate (blue curve) has a silighly higher absorption than the other two kinds of substrate. It has the highest absorption of $80 \%$ at $400 \mathrm{~nm}$ and generally higher absorption in the 550 to $700 \mathrm{~nm}$ range. The nanostructuring may give light traping benefits and enhance the absorption.

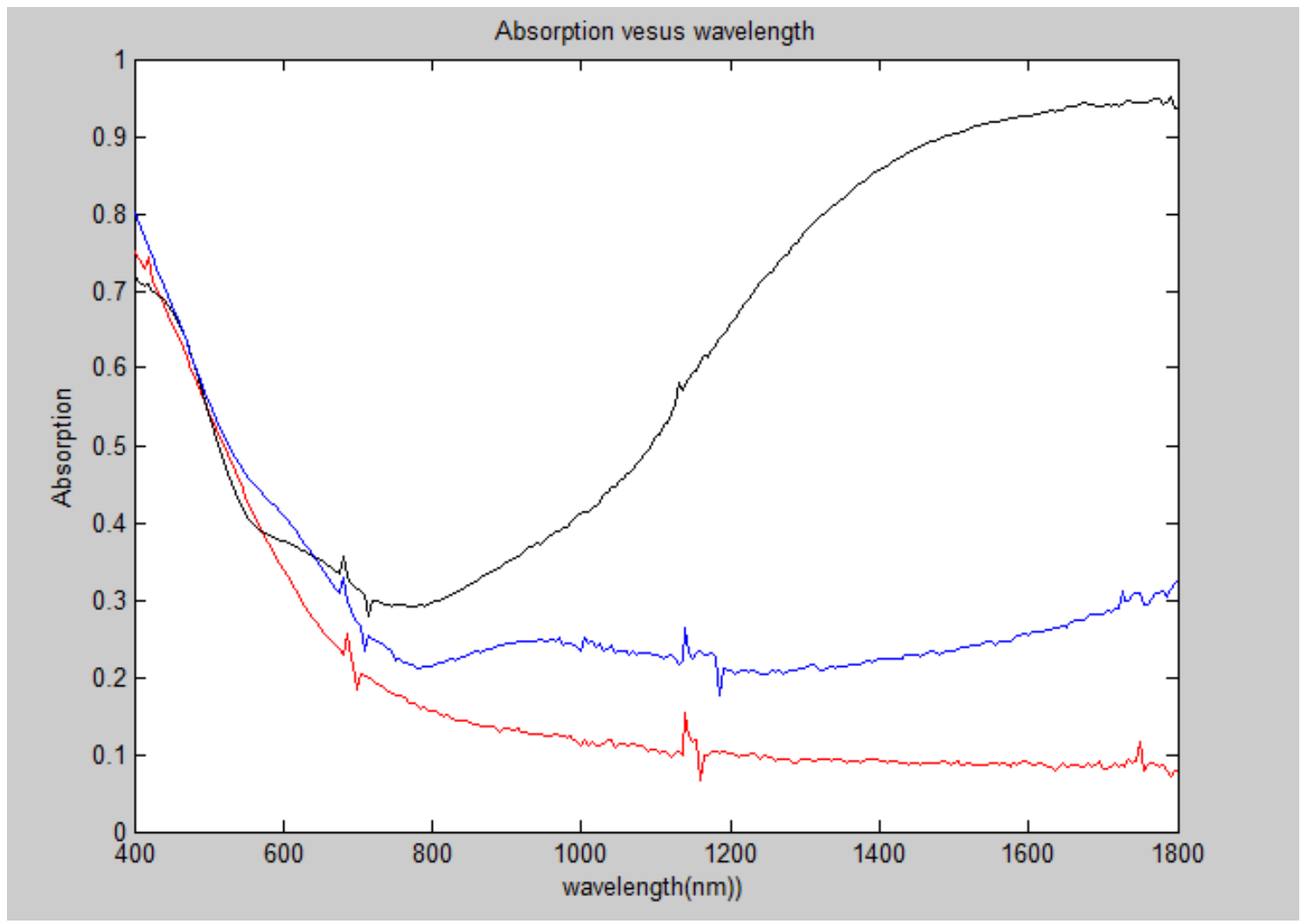

Figure 5.4Absorption of a-Si on different substrate (Glass: red curve; ITO: black curve; Nano-structured ITO: blue curve) 
However, due to the difference in free carrier concentration, they all have a unique absorption behavior for wavelengths greater than $1000 \mathrm{~nm}$. The glass (red curve), which does not have the free electron absorption, has a relative constant low absorption from 1000 to $1800 \mathrm{~nm}$. The nanostructured ITO has higher free carrier absorption than glass but lower than the ITO glass (the black curve). The ITO glass with a-Si film has a huge absorption after 1000nm due to the free carrier absorption.

\subsubsection{8nm a-Si film vs 4nm a-Si film}

To examine the effect of a-Si film thickness, the absorption of 4nm and 8nm a-Si films on a nanostructured ITO substrate are shown in Figure 5.5. One would expect the 4nm film to absorb at least $50 \%$ as much light as the $8 \mathrm{~nm}$ film, but Fig. 5.5 shows that the $4 \mathrm{~nm}$ film absorbs far less than this. Indeed, in the 400 to $800 \mathrm{~nm}$ range the absorption is only slightly greater than that of the structured ITO substrate itself. The film thickness of $4 \mathrm{~nm}$ and $8 \mathrm{~nm}$ were estimated on the basis of known deposition rates for the thicker, easily measurable films. It appears the $4 \mathrm{~nm}$ film may be much thinner than expected perhaps due to the need for an incubation time before film growth begins. 


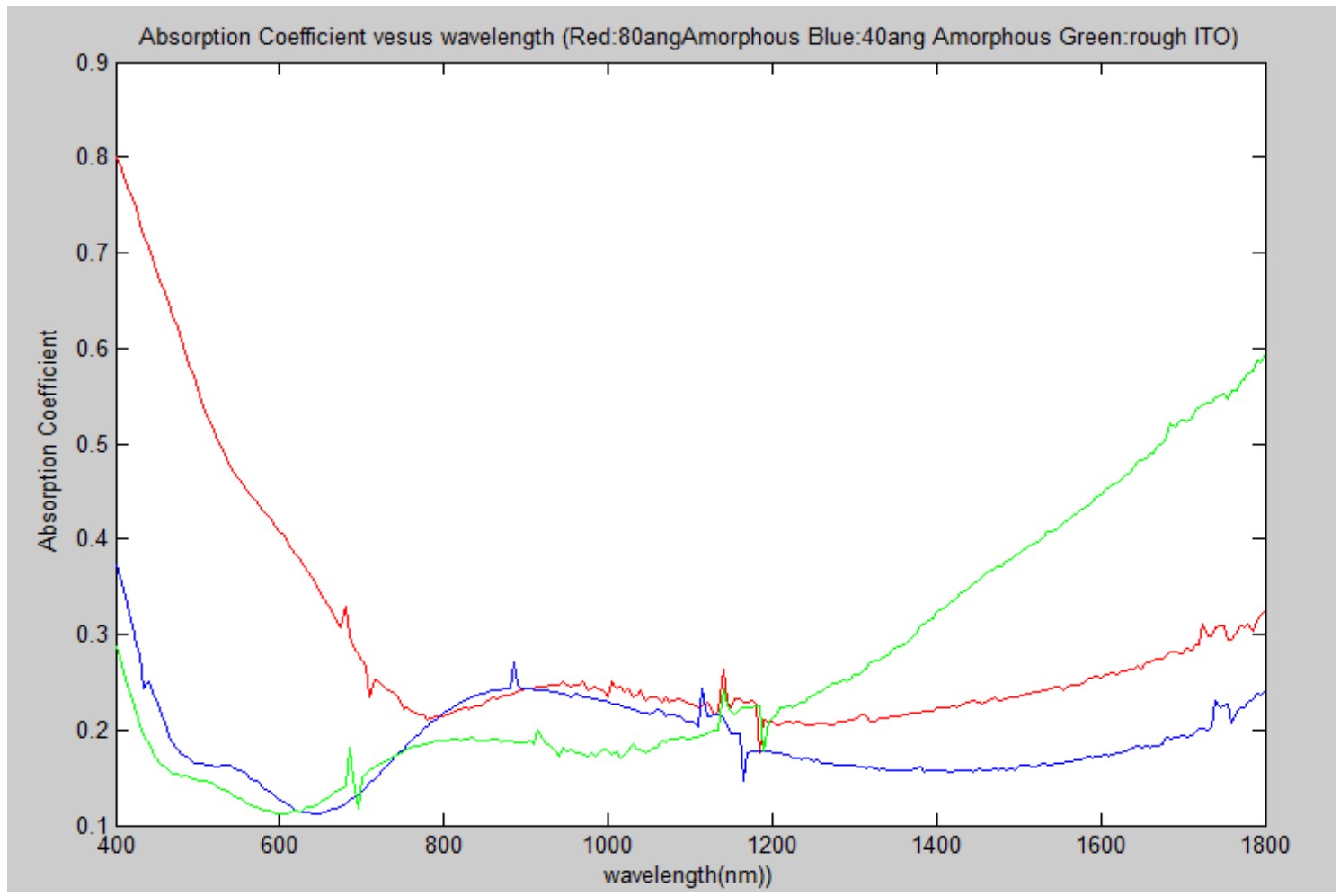

Figure 5.5: Absorption of $4 \mathrm{~nm}, 8 \mathrm{~nm}$ amorphous film and ITO sample (4nm film: blue curve; Nanostructured ITO glass: green curve; 8nm silicon film: red curve)

\subsubsection{8nm amorphous silicon film vs 8nmcrystallized silicon film}

The $8 \mathrm{~nm}$ thick amorphous silicon film was crystallized at $600^{\circ} \mathrm{C}$ for 60 minutes. Fig. 5.6 compares the absorption of crystallized and annealed films. The crystallized silicon (black curve) has a lower absorption than the amorphous silicon (red curve) between 400 and 800nm. Over this range the absorption of the amorphous silicon is $10 \%$ higher than the crystallized silicon. However, in the wavelength above $1000 \mathrm{~nm}$, the absorption of c-Si is much higher than the a-Si film. That is because there are more free carrier absorption in the c-Si film. 


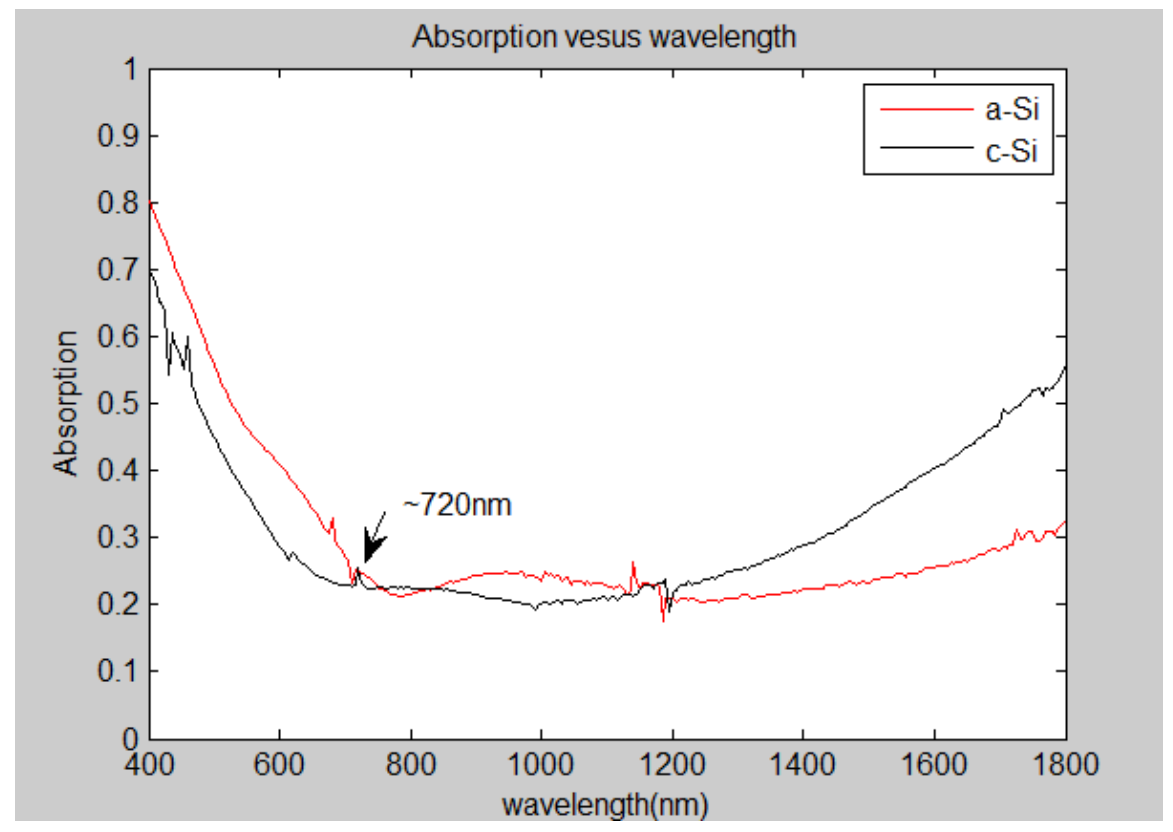

Figure 5.6Amorphous silicon vscrystallized silicon

The bandgap of the crystallized silicon film appears to be very similar to the bandgap of the amorphous film. In fact, both c-Si and a-Si has absorption edge at around $720 \mathrm{~nm}(1.72 \mathrm{eV})$. This energy is higher than the bulk c-Si $(1.09 \mathrm{eV})$ mentioned in Chapter 2. Also, comparing with the aSi deposited on glass as shown in Figure 5.1, 720nm is quite close to the $750-800 \mathrm{~nm}$ range. Therefore we can conclude that the a-Si deposited on glass has the same bandgap as the a-Si deposited on nanostrustured ITO. Although the crystallization does not appear to alter the bandgap of the film, it decreases the absorption in the $400-1200 \mathrm{~nm}$ range and increases the absorption in the $1200-1800 \mathrm{~nm}$ range. 


\subsection{Reflection and Refractive Index Analysis}

It is possible to find both the real part and the imaginary part of refractive index of a film by measuring the amplitude and phase of the reflection coefficient. Then, the absorption coefficient can be calculated according to the formula:

$$
\alpha=\frac{4 \pi \mathrm{k}}{\lambda}(5.1)
$$

where $\lambda$ is the wavelength, and $\mathrm{k}$ is the imaginary part of the refractive index.

By studying the dependence of the absorption coefficient on wavelength, we can find the bandgap for the film.

First, the reflection and refractive index of the uncoated Nano-structured ITO are discussed. Second, the nano-structured ITO with 8nm thick amorphous silicon film is analyzed in detail. Finally, a 4nm thick amorphous silicon film and a crystallized silicon film are compared with the 8nm thick amorphous silicon film. Overall, the goal of this analysis is to determine the bandgap and the absorption coefficient of the thin silicon film. 


\subsubsection{Analysis of uncoated Nano-structured ITO}

\subsubsection{Reflection}

Although the ITO has a bandgap of about $4 \mathrm{eV}$, the free carrier absorption is still significant for wavelengths above $1000 \mathrm{~nm}$. As a result, it is important to analyze the absorption influence from the nano-structured ITO substrate. Fig.5.7 and Fig. 5.8 show the reflectance of the ITO film, which $\mathrm{t}$ is less than $15 \%$ in all wavelengths. It has a peak point at around $800 \mathrm{~nm}$.

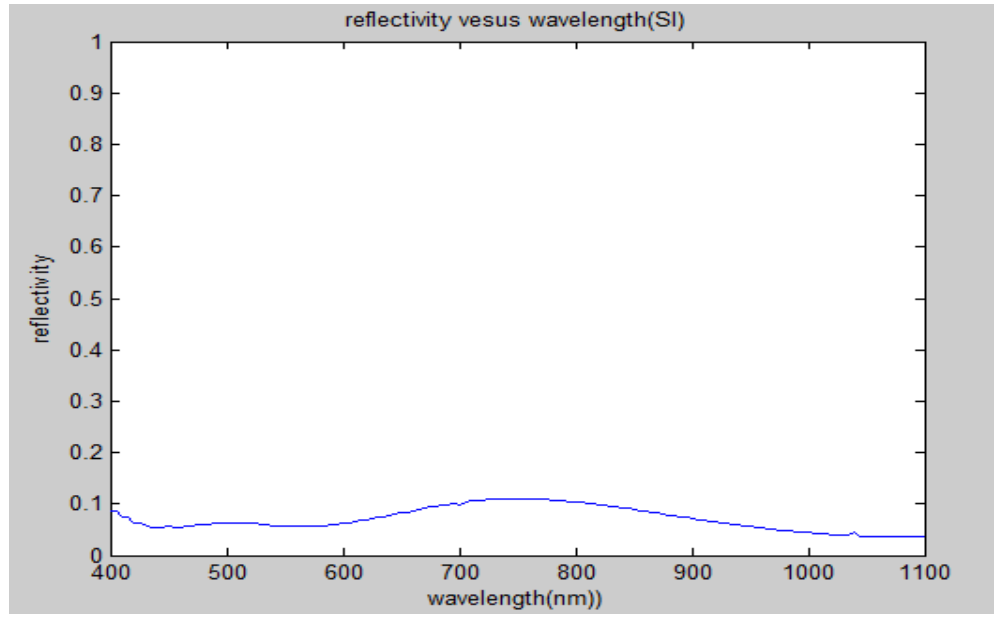

Figure 5.7Reflectance of uncoated nanostructured ITO (Si detector)

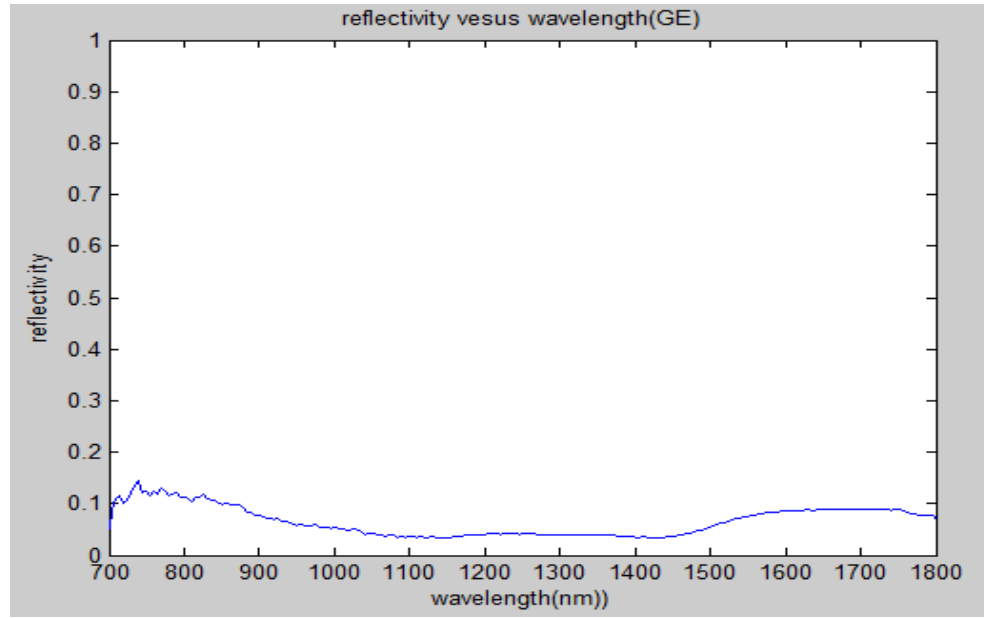

Figure 5.8Reflectance of uncoated nanostructured ITO (Ge detector) 


\subsubsection{Refractive Index}

As mentioned in Chapter 2, the Kramers- Kronig equation allows extraction of the real and imaginary part of the refractive index from the reflectance. The refractive index of the ITO film was determined and is shown in Figure 5.9- Figure 5.12. In Fig. 5.9 and Fig. 5.10, the real part n fluctuates slightly at a value around 1.9 from $400 \mathrm{~nm}$ to1800nm wavelength.

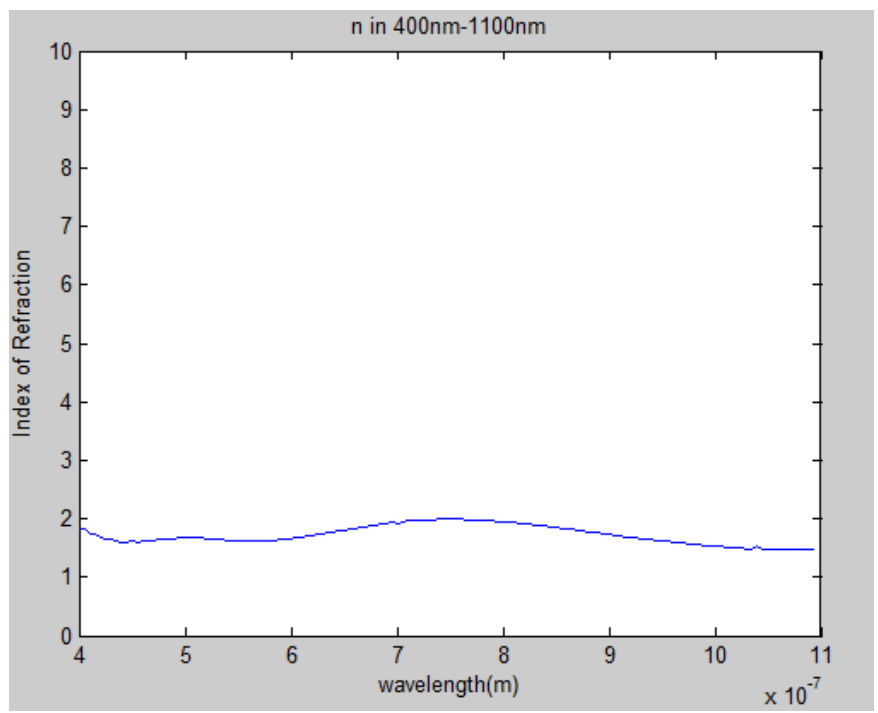

Figure 5.9Real part of refractive index (Si detector)

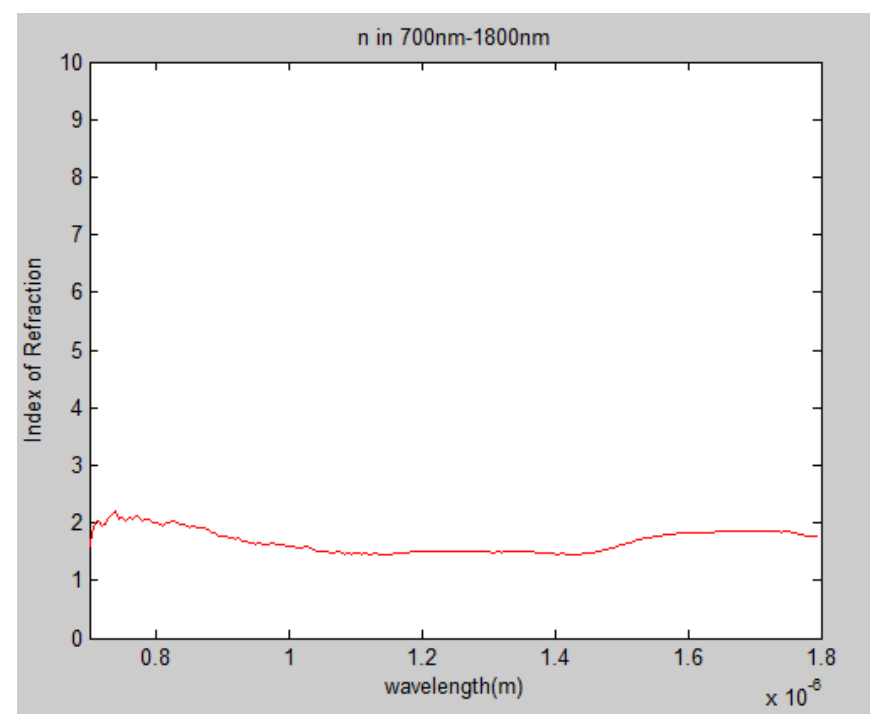

Figure 5.10Real part of refractive index (Ge detector) 
The imaginary part of the refractive index is also called the extinction coefficient $\mathrm{k}$, and describes the amount of absorption loss when the electromagnetic wave propagates through the film. In Figure 5.11, the extinction coefficient of the ITO film was obtained from 400nm to $1800 \mathrm{~nm}$. The extinction coefficient stays 0 between $400 \mathrm{~nm}$ and $765 \mathrm{~nm}$. After that, the extinction goes positive from $765 \mathrm{~nm}$ to $1330 \mathrm{~nm}$. Finally, the extinction coefficient goes back to effectively zero again. In some wavelengths the extinction coefficient goes below zero. This does not mean optical gain and it is caused by light loss during the reflection measurements. The negative value can be seen as zero. Overall, the ITO film absorbs light weakly over the $765 \mathrm{~nm}$ to $1330 \mathrm{~nm}$ wavelength.

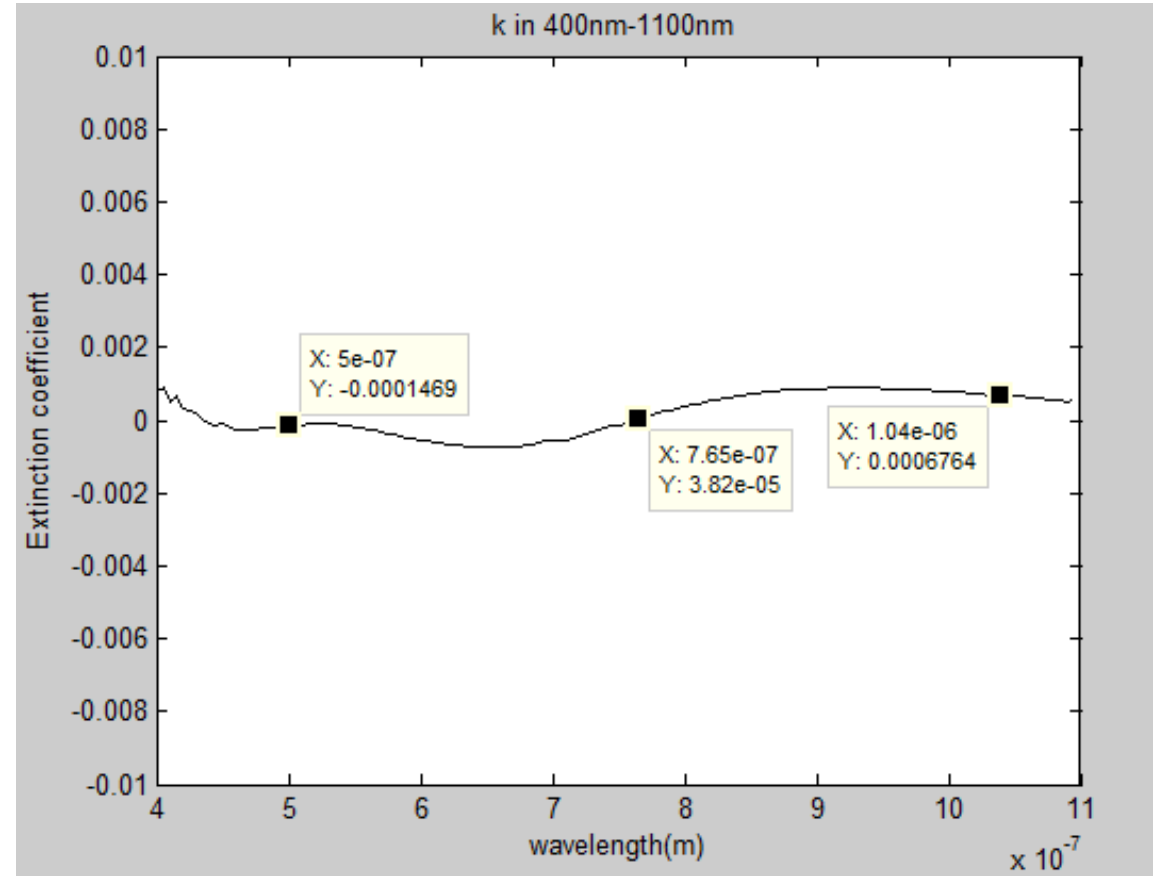

Figure 5.11/maginary part of refractive Index (Si detector) 


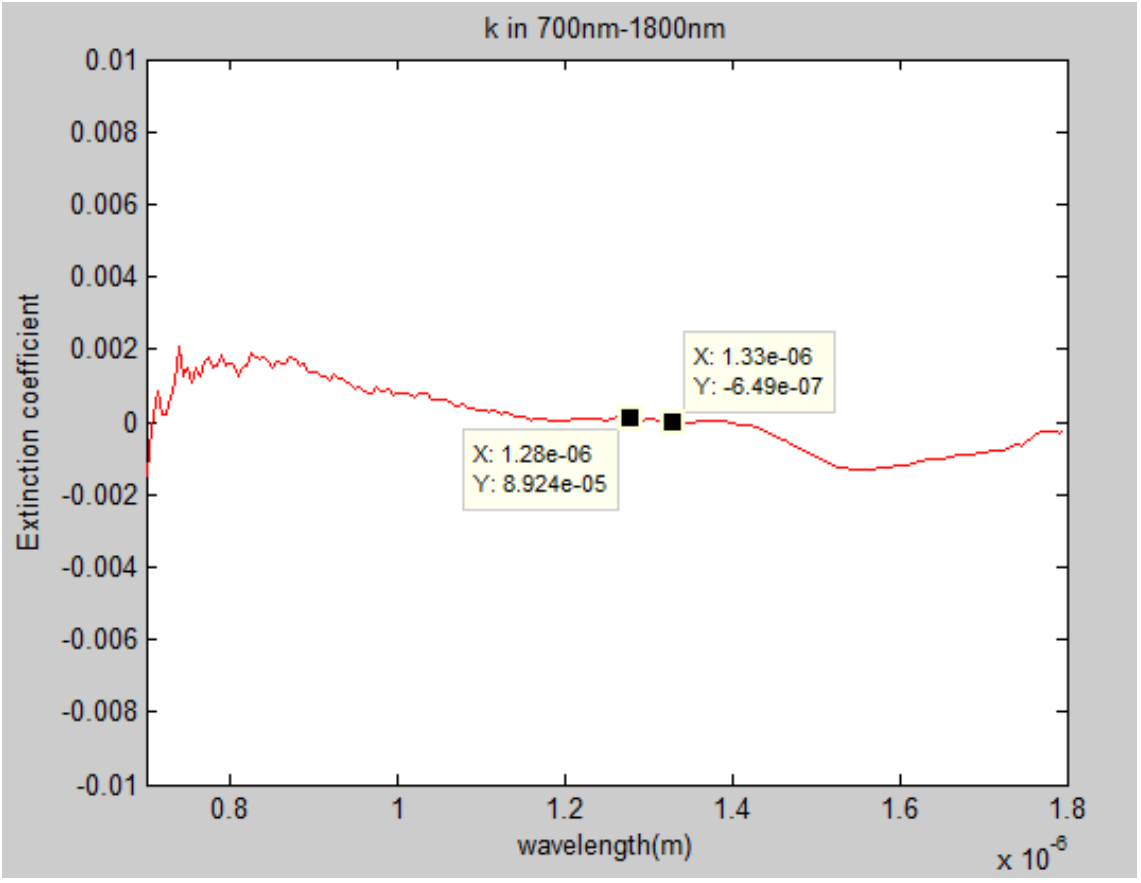

Figure 5.12Imaginary part of refractive index (Ge detector) 


\subsubsection{8nmthick amorphous silicon deposited on Nano-structured ITO}

\subsubsection{Reflection}

The reflection of the $8 \mathrm{~nm}$ thick amorphous silicon film on nano-structured ITO was measured using the silicon detector from $400 \mathrm{~nm}$ to $1100 \mathrm{~nm}$ and the germanium detector from $1100 \mathrm{~nm}$ to $1800 \mathrm{~nm}$. As shown in Figure 5.13, the film has $34.48 \%$ reflection at $400 \mathrm{~nm}$, decreasing to $20.8 \%$ at $600 \mathrm{~nm}$. However, the reflection tends to be relatively stable between $800 \mathrm{~nm}(10.61 \%)$ and $1000 \mathrm{~nm}(9.375 \%)$.

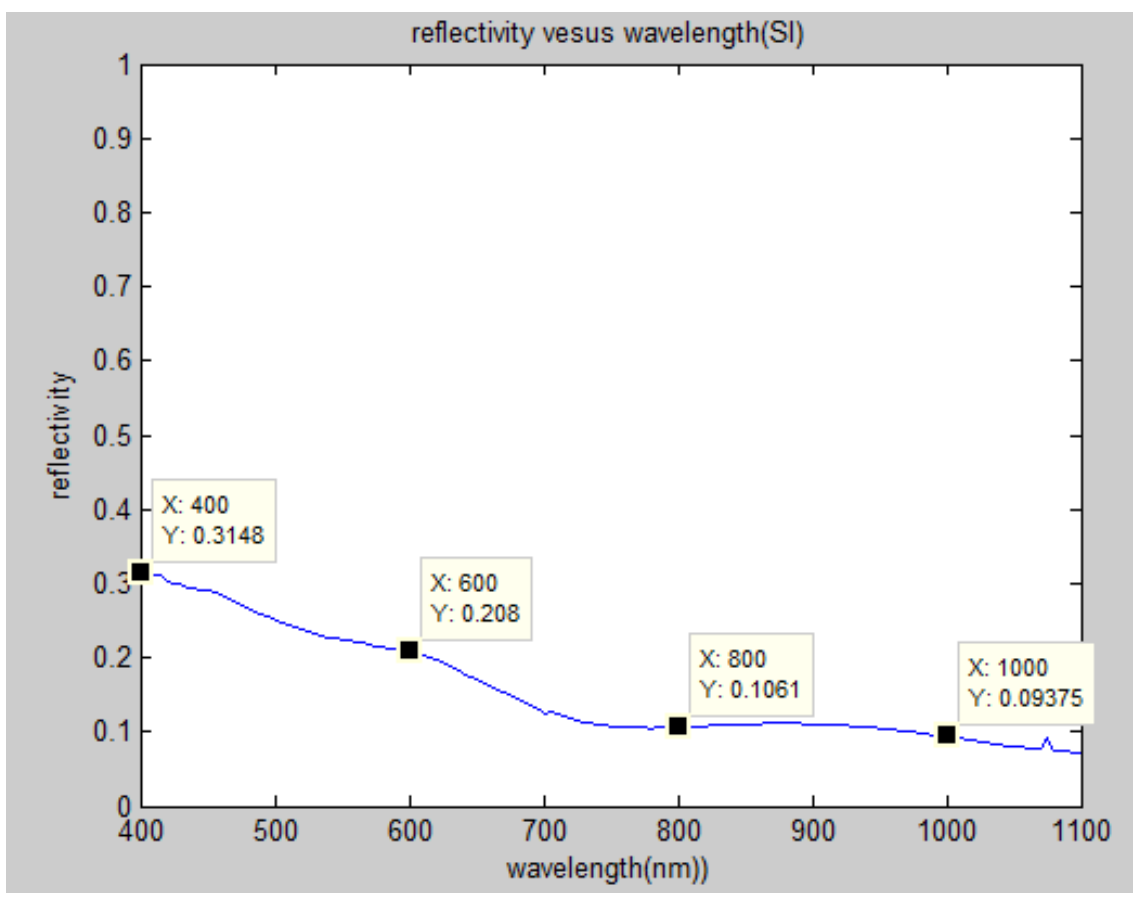

Figure 5.13Reflection of $8 \mathrm{~nm}$ a-Si film for 400-1100nm wavelengths 
The reflection for wavelengths greater than 1000nm is shown in Figure 5.14. The reflection varies around $8.5 \%$. Compared with the shorter wavelength, the reflectance of the film stays fairly steady after $800 \mathrm{~nm}$ at around $10 \%$.

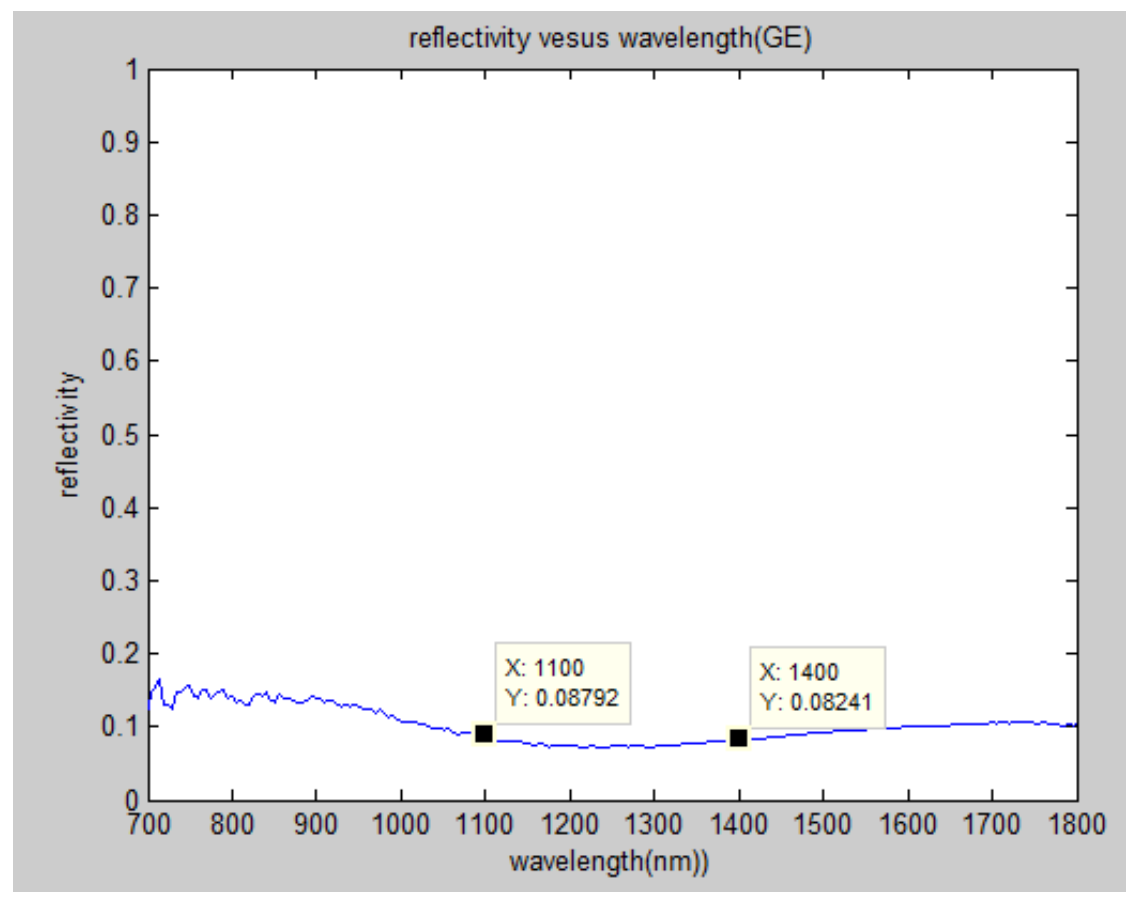

Figure 5.14Reflection of $8 \mathrm{~nm}$ a-Si film for 1100-1800nm wavelengths

\subsubsection{Thin Film interference}

Since the samples are made by depositing very thin amorphous silicon films on a glass substrate, the thin film interference will affect the reflection performance of the sample. An important consideration in determining whether these waves interfere constructively or destructively is the fact that whenever light reflects off a surface of higher index of refraction, a $180^{\circ}$ phase shift in the wave is introduced. In our sample, the bottom substrate is glass with $n_{\text {glass }}=1.5$ and the top 
layer is a-Si with $\mathrm{n}_{\mathrm{aSi}}=3.96$ approximately and the air has $\mathrm{n}_{\mathrm{air}}=1$. There will be $180^{\circ}$ phase shift since $\mathrm{n}_{\mathrm{air}}<\mathrm{n}_{\mathrm{aSi}}$ but $\mathrm{n}_{\mathrm{aSi}}>\mathrm{n}_{\text {glass }}$. Another consideration is that the thickness of the thin film must satisfy the following equation to either be constructive or destructive reflection:

$$
\begin{aligned}
& d=\frac{\cos (\theta)}{2 n_{\text {film }}} m \lambda \text { for destructive interference }(5.2) \\
& d=\frac{\cos (\theta)}{2 n_{\text {film }}}\left(m+\frac{1}{2}\right) \lambda \text { for constructive interference }(5.3)
\end{aligned}
$$

where :
$\mathrm{d}$ is the film thickness
$\theta$ is the incidence angle
$m$ is just an integer
$\mathrm{n}_{\text {film }}$ is the top film refractive index
$\lambda$ is the wavelength of the light.

In the experiment, the light source has a range of $400 \mathrm{~nm}$ to $1800 \mathrm{~nm}$ and $\mathrm{n}_{\text {film }}=\mathrm{n}_{\mathrm{aSi}}=$ $5.6 \sim 3.5$.

At normal incident, $\mathrm{d}$ can be solved as following:

For destructive at $400 \mathrm{~nm}: \mathrm{d}=\frac{\cos (\theta)}{2 \mathrm{n}_{\text {film }}} \mathrm{m} \lambda=\frac{1}{2 * 5.6} * \mathrm{~m} * 400 \mathrm{~nm}=35.7,72.42,107.1, \ldots$

For destructive at $1800 \mathrm{~nm}: \mathrm{d}=\frac{\cos (\theta)}{2 \mathrm{n}_{\mathrm{film}}} \mathrm{m} \lambda=\frac{1}{2 * 3.5} * \mathrm{~m} * 1800 \mathrm{~nm}=257,514,771 \mathrm{~nm}, \ldots$ 
For constructive at 400nm:

$$
\mathrm{d}=\frac{\cos (\theta)}{2 \mathrm{n}_{\mathrm{film}}}\left(\mathrm{m}+\frac{1}{2}\right) \lambda=\frac{1}{2 * 5.6} *\left(\mathrm{~m}+\frac{1}{2}\right) * 400 \mathrm{~nm}=53.5,89.2,124.9 \mathrm{~nm}, \ldots
$$

For constructive interference at $1800 \mathrm{~nm}$ :

$$
\mathrm{d}=\frac{\cos (\theta)}{2 \mathrm{n}_{\mathrm{film}}}\left(\mathrm{m}+\frac{1}{2}\right) \lambda=\frac{1}{2 * 3.5} *\left(\mathrm{~m}+\frac{1}{2}\right) * 1800 \mathrm{~nm}=385,642,899 \mathrm{~nm}, \ldots
$$

In all the calculation, the thickness $\mathrm{d}$ is at least $35 \mathrm{~nm}$ thick. Compared to the a-Si thickness $8 \mathrm{~nm}$, the thin film interference will not occur over $400 \mathrm{~nm}$ to $1800 \mathrm{~nm}$ range.

\subsubsection{Refractive Index of $8 \mathrm{~nm}$ film}

According to the Kramers-Kronig equation, the real part and imaginary part of the refractive index can be calculated from the reflectance. The results were shown in the following Fig.

\subsection{5and Fig.5.16.}

In Figure 5.11, the real part of the refractive index was calculated from 400nm to $1000 \mathrm{~nm}$ and $1100 \mathrm{~nm}$ to $1800 \mathrm{~nm}$. The $8 \mathrm{~nm}$ thick amorphous silicon film has $\mathrm{n}=3.557$ at $400 \mathrm{~nm}$ and the value decreases to 1.966 at $800 \mathrm{~nm}$. Also, at the wavelength above $1100 \mathrm{~nm}$, the refractive index stays steady around 1.8 . 


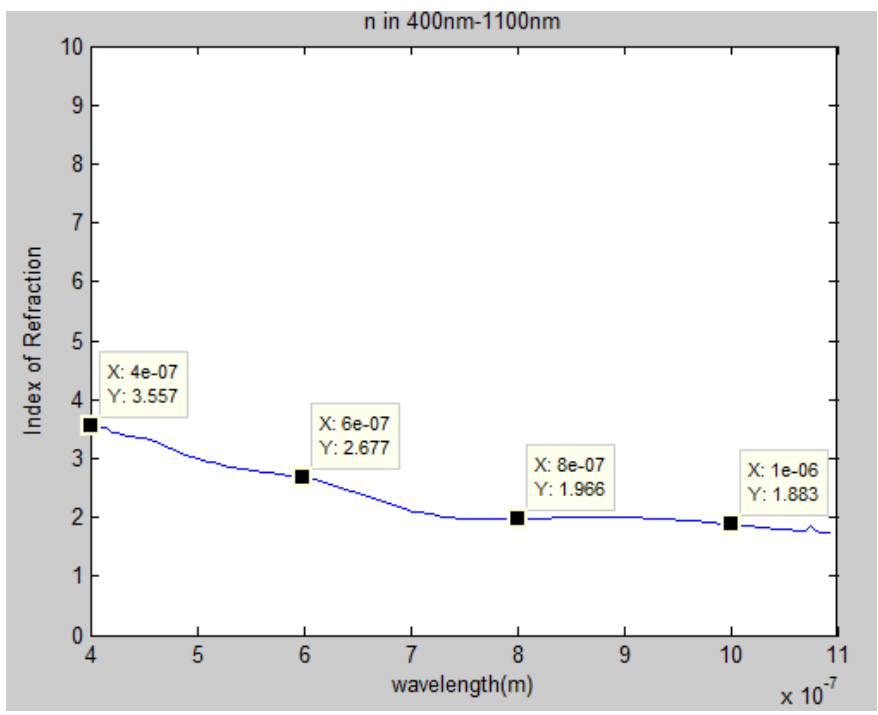

Figure 5.15The real part of the refractive index 400-1100nm

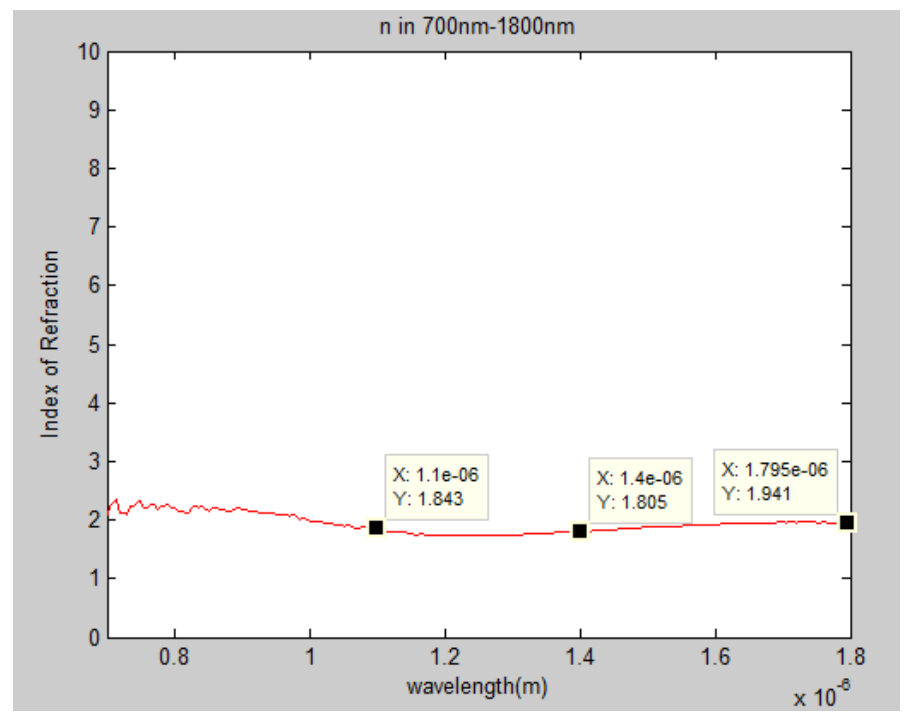

Figure 5.16 The real part of the refractive index700-1800nm

A comparison of the $8 \mathrm{~nm}$ a-Si refractive index with the known refractive index of amorphous silicon in Chapter 2 is shown in Fig 5.17. The blue curve is the refractive index data from M.A. Green[24].The calculated 8nm refractive index values are much smaller than the known a-Si 
refractive index. The reason is that the sample is far less dense than conventional a-Si, the "tree" like structure provides huge amount of empty space.

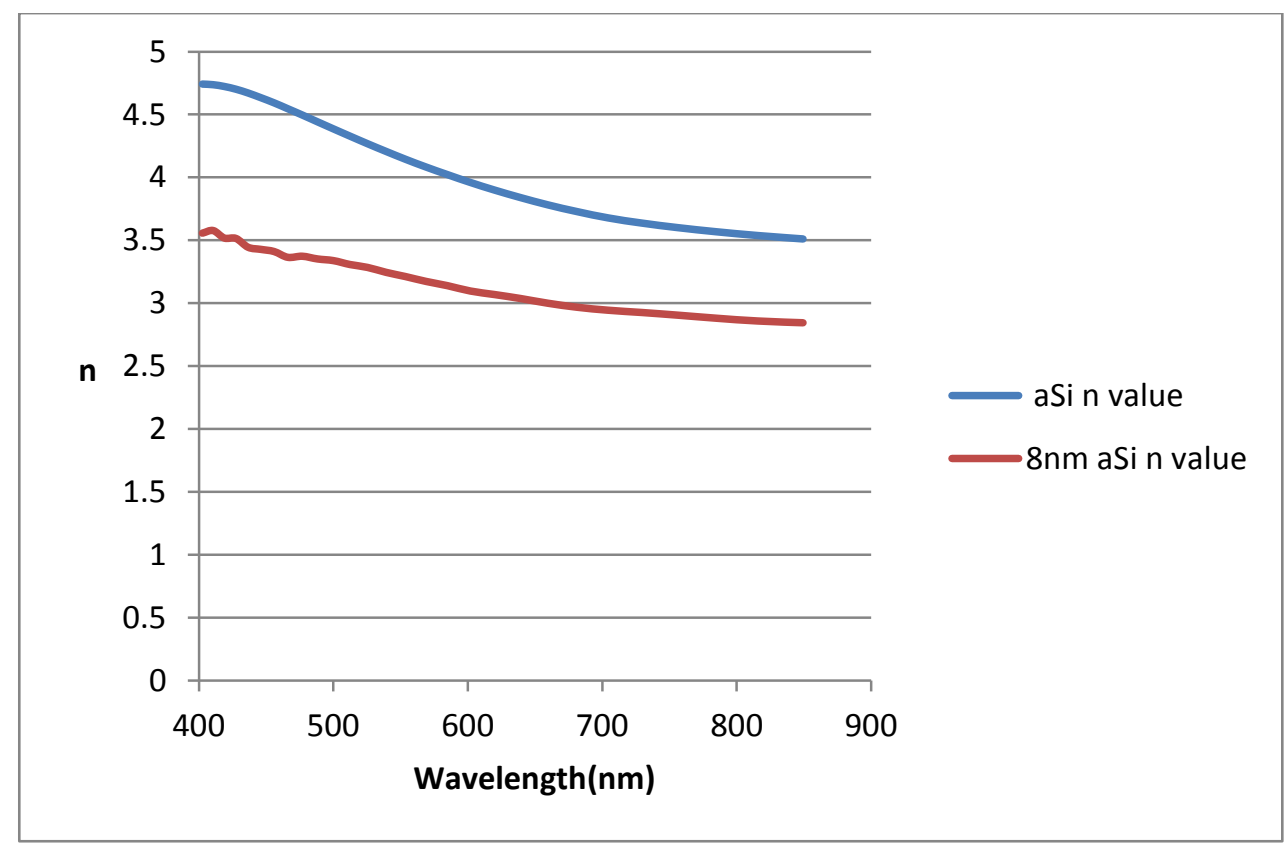

Figure 5.16Bulk a-Si n vs 8nm a-Si film n

The imaginary part or the extinction coefficient of the film is shown in Fig. 5.18. It started from $3.593 \mathrm{E}-3$ at $400 \mathrm{~nm}$ and decreased to $9.005 \mathrm{E}-4$ at $800 \mathrm{~nm}$. After $800 \mathrm{~nm}$, the extinction coefficient turns stabilizes at 9.005E-4. However, the extinction coefficient decreased to 0 at $1335 \mathrm{~nm}$ in Fig. 5.19, which indicates the light will not be absorbed after this wavelength. This is unusual for amorphous silicon as the bandgap is around $800 \mathrm{~nm}$. The absorption between $800 \mathrm{~nm}$ and $1335 \mathrm{~nm}$ is attributed to the nano-structured ITO substrate. As mentioned in Section 5.3.1.2, the ITO structured absorbs a small amount of light in the 765 to $1330 \mathrm{~nm}$ range. 


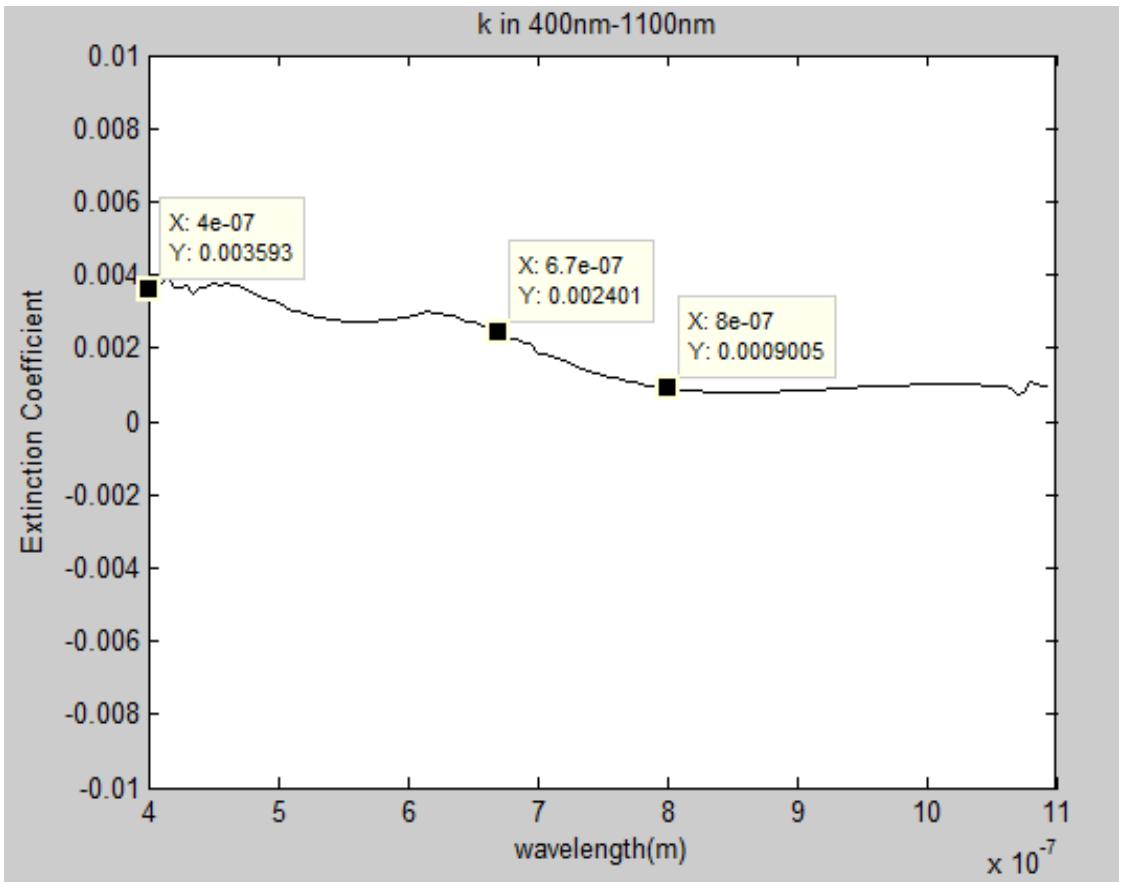

Figure 5.17 The imaginary part of refractive index $400-1100 \mathrm{~nm}$

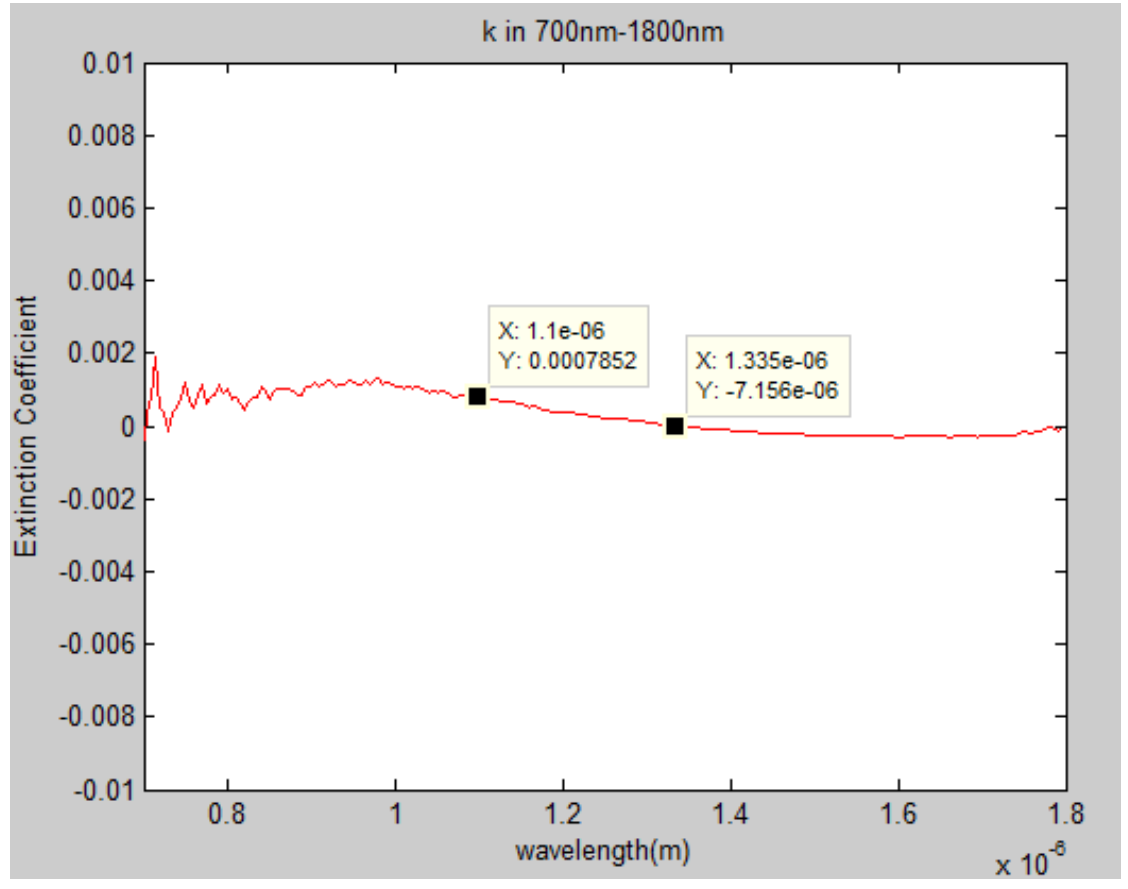

Figure 5.18 The imaginary part of refractive index $700-1800 \mathrm{~nm}$ 


\subsubsection{Absorption Coefficient}

The absorption coefficient was calculated based on the extinction coefficient $\mathrm{k}$. The results are shown in Fig. 5.20 and Fig. 5.21. The absorption coefficient begins with $10^{3} \mathrm{~cm}^{-1}$ at $400 \mathrm{~nm}$ and decreases to $10^{2} \mathrm{~cm}^{-1}$ at $800 \mathrm{~nm}$. As the wavelength increases further, the absorption coefficient stays steady from $800 \mathrm{~nm}$ to $1100 \mathrm{~nm}$ and then drops to 0 at $1300 \mathrm{~nm}$. However, the absorption coefficient after $800 \mathrm{~nm}$ is as a result of free carrier absorption of ITO film. This is why the coefficient stays steady from $800 \mathrm{~nm}$ to $1100 \mathrm{~nm}$. The real bandgap of the a-Si film is at $825 \mathrm{~nm}$ or $1.5 \mathrm{eV}$ where the absorption coefficient stays steady.

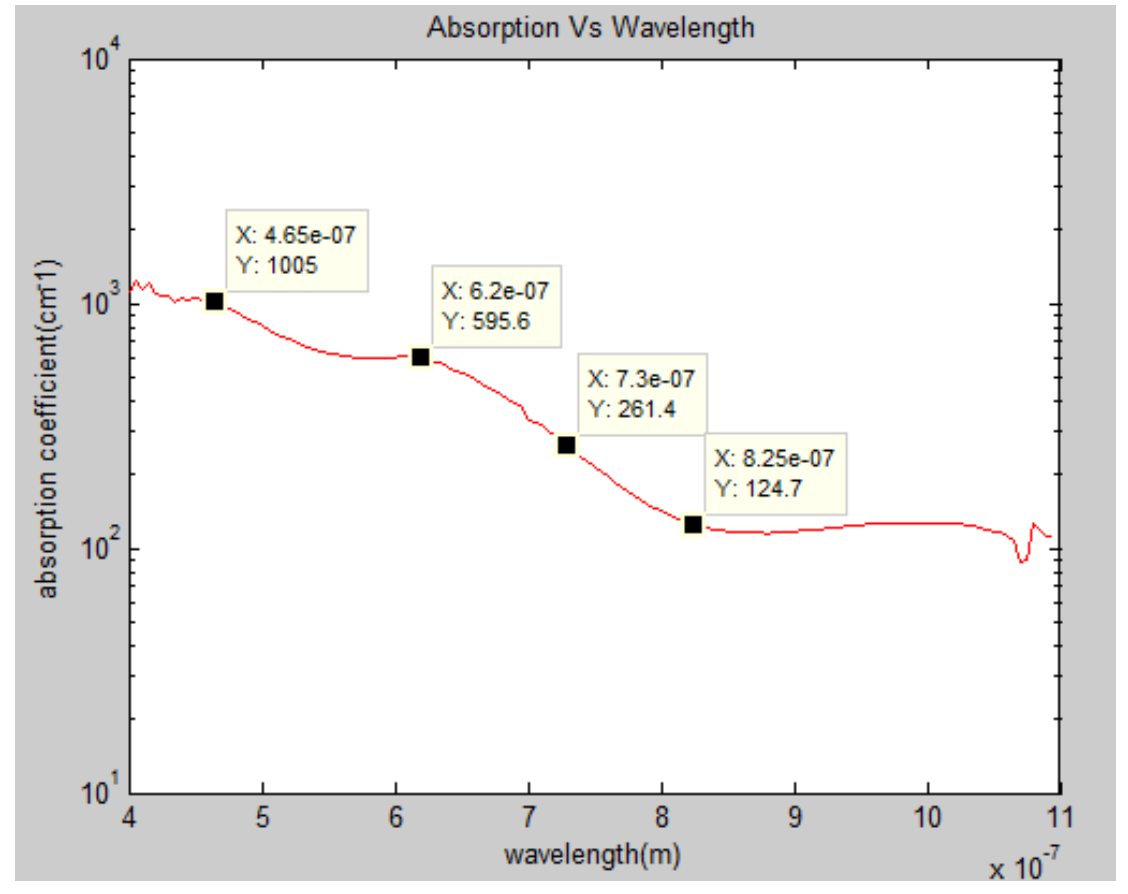

Figure 5.19 Calculated absorption coefficient (Si detector) 


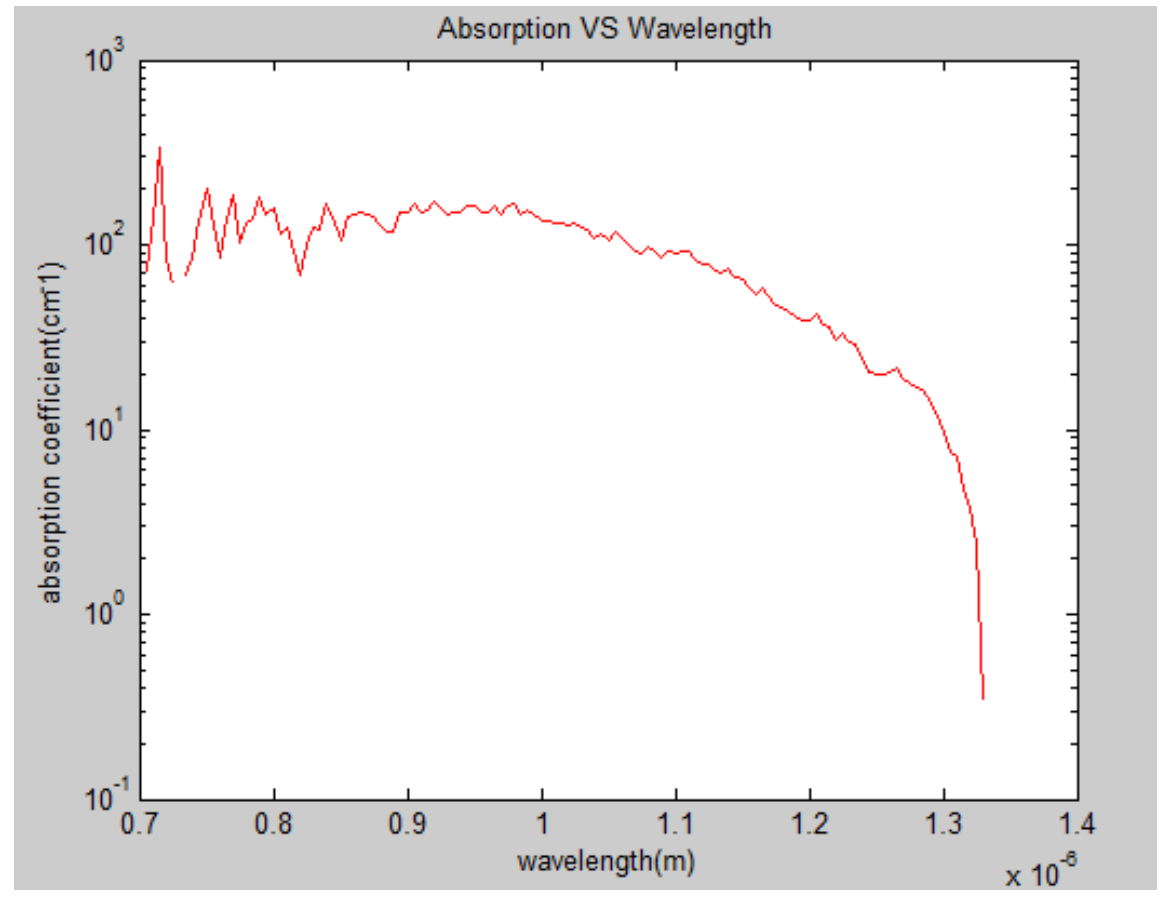

Figure 5.20 Calculated absorption coefficient (Ge detector)

\subsubsection{8nm thick crystallized silicon deposited on Nano-structured ITO}

The amorphous silicon film was crystallized to study the absorption coefficient difference between a-Si and c-Si. In Figure 5.22 and Figure 5.23, the absorption coefficient of the crystallized silicon is plotted. The bandgap of the c-Si film can be determined at $1125 \mathrm{~nm}$ or $1.1 \mathrm{eV}$ where the absorption coefficients decreased to zero. Comparing the a-Si with c-Si, the a-Si has higher absorption coefficient from $400 \mathrm{~nm}$ to $800 \mathrm{~nm}$, almost double that of the c-Si film. However, c-Si has higher absorption performance from $800 \mathrm{~nm}$ to $1100 \mathrm{~nm}$. 


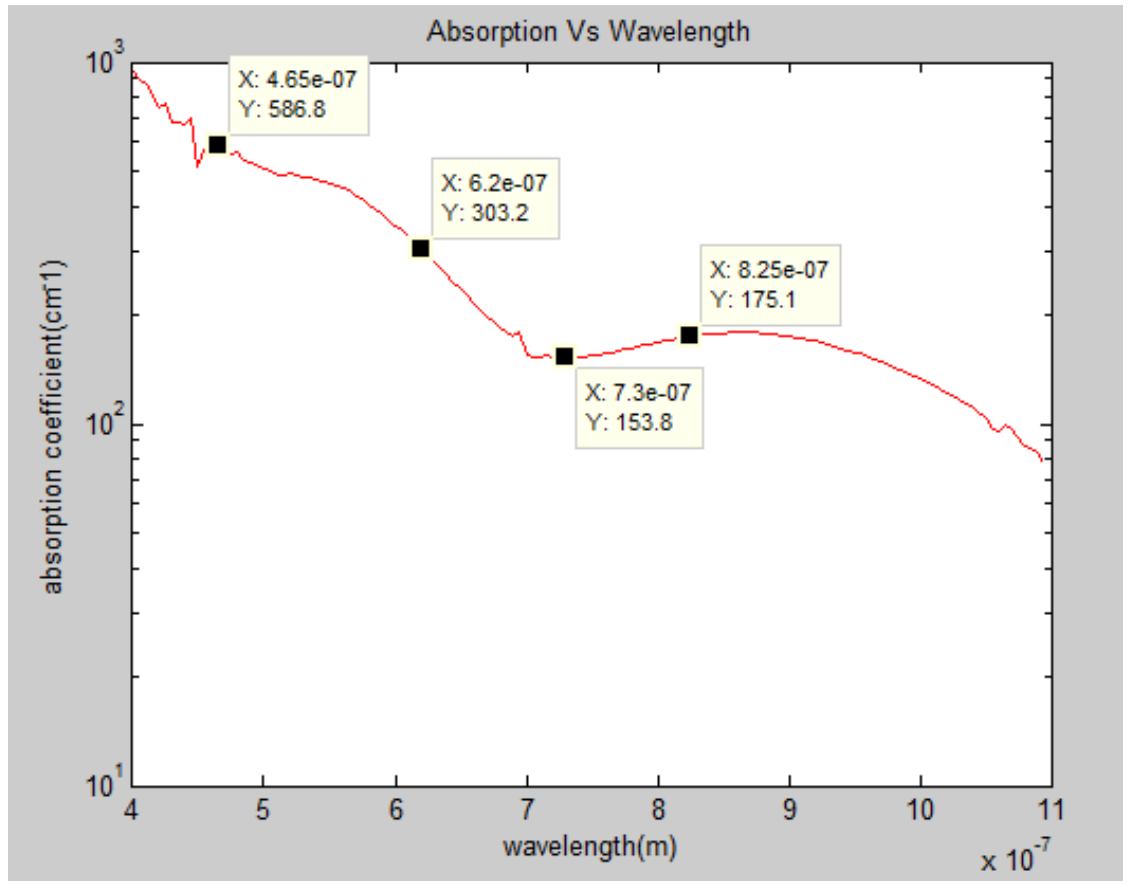

Figure 5.21 Absorption coefficient (Si detector)

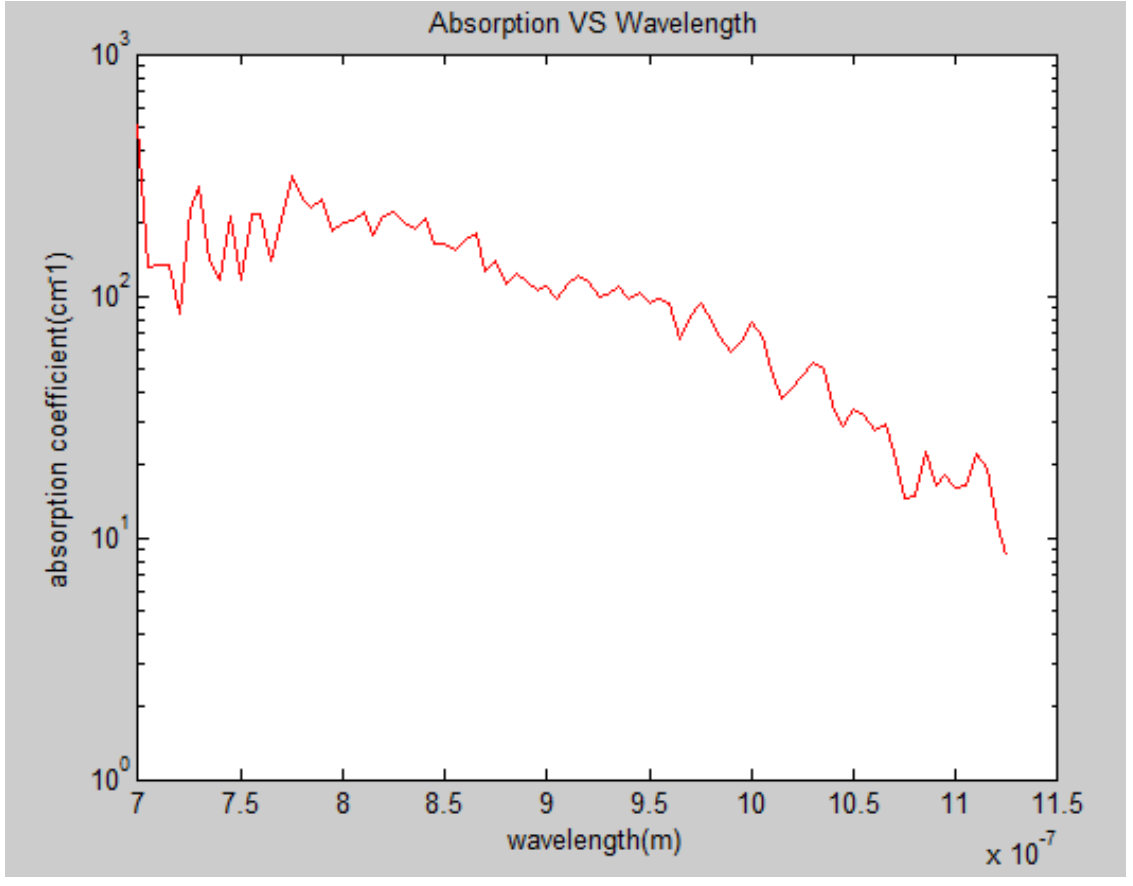

Figure 5.22Absorption coefficients (Ge detector) 


\subsubsection{4nm thick amorphous silicon deposited on nano-structured ITO}

The previous results were for $8 \mathrm{~nm}$ thick amorphous silicon films. In order to study the influence of film thickness, the reflectance of a $4 \mathrm{~nm}$ silicon film was measured. The absorption coefficient is extracted and shown in Fig 5.24 and Fig5.25. The absorption coefficients are zero from 450nm to $950 \mathrm{~nm}$ and stay positive in the rest of the range. This is totally different with the $8 \mathrm{~nm}$ film. In fact, the absorption coefficients of this film are more like the ITO glass. This also verifies that the $4 \mathrm{~nm}$ film is too thin for observing the bandgap of amorphous silicon.

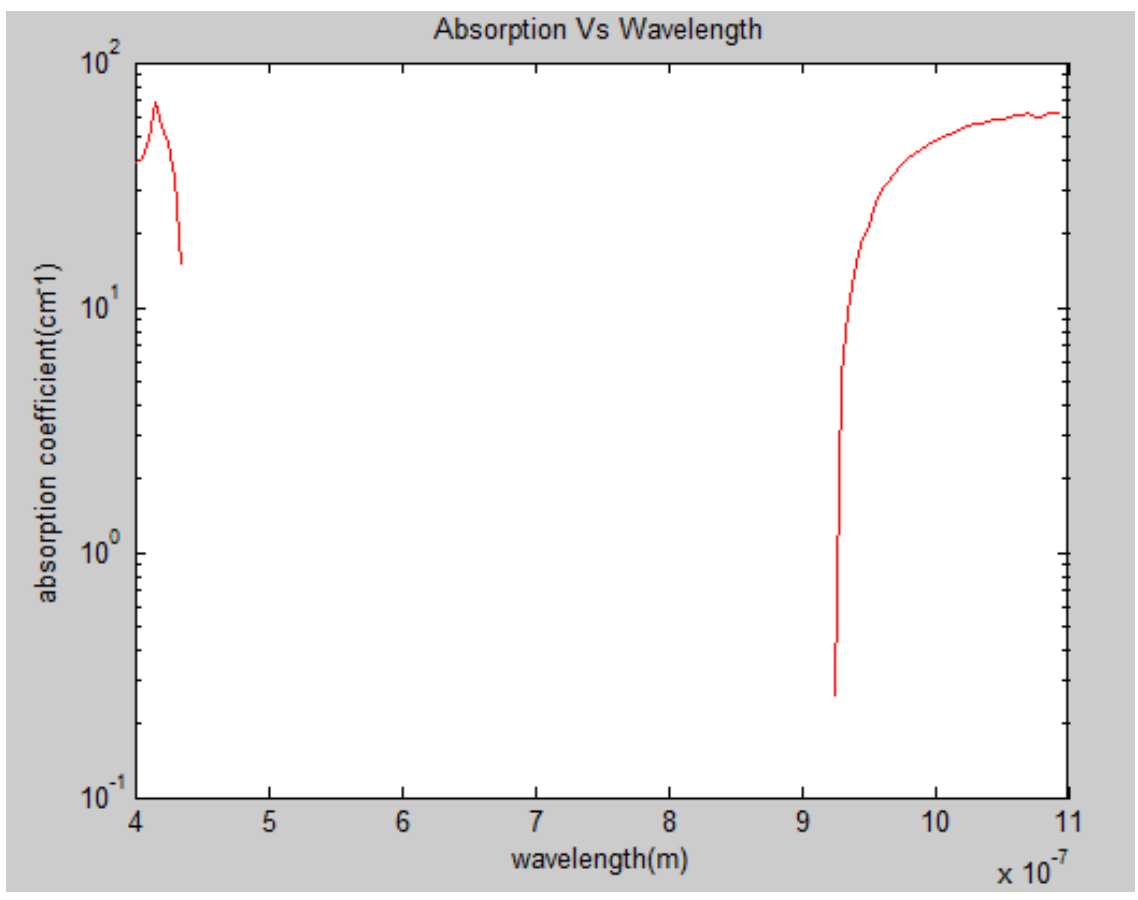

Figure 5.23 Absorption coefficient (Si detector) 


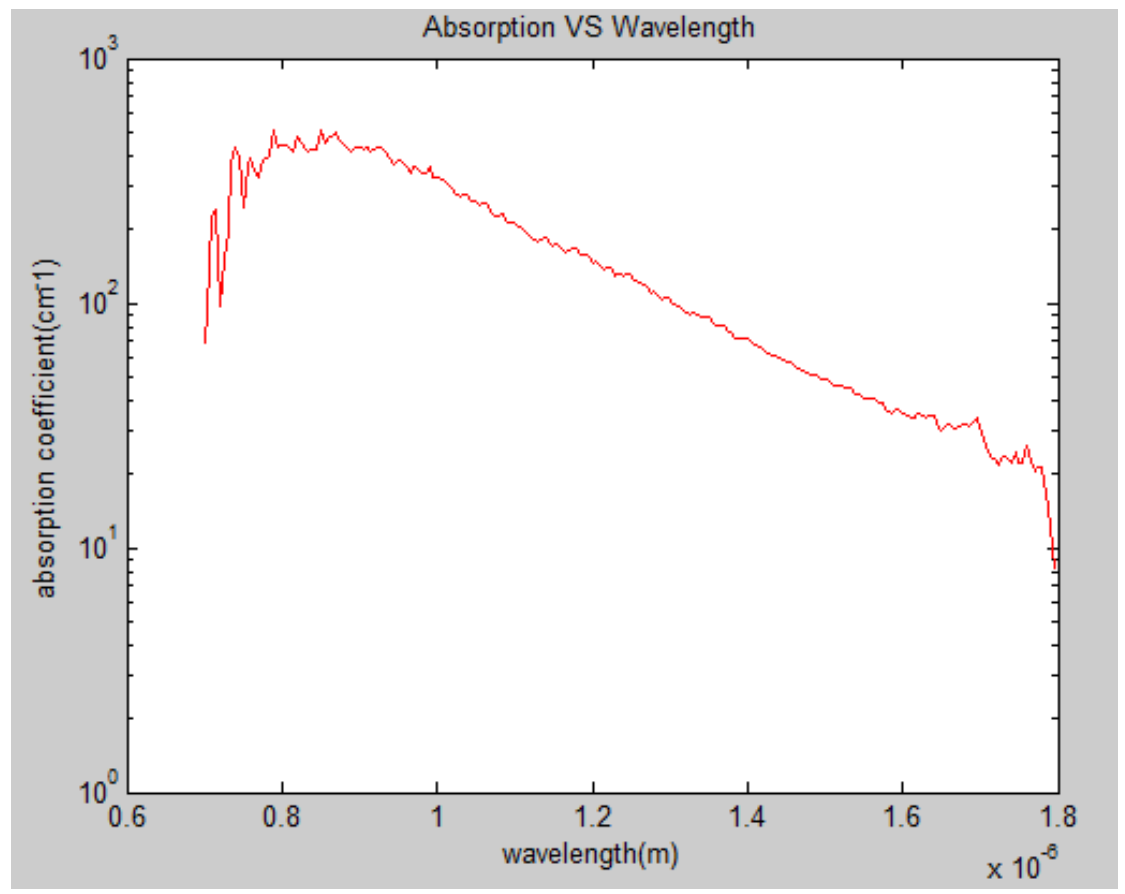

Figure 5.24Absorption coefficients (Ge detector)

\subsection{Solar spectrum absorption}

Since the deposition of our film is a solar cell application, it is crucial to estimate how much of the incident solar spectrum is absorbed in this film. The absorption of a-Si and c-Si film was plotted together with solar spectral irradiance in Fig 5.26 and Fig 5.27. To calculate the energy absorption respective to solar spectrum, we will multiply the spectral irradiance with absorption at each wavelength first. Then, find the sum of the absorbed energy and divide by the sum of the solar irradiance. As estimated, the a-Si has overall solar spectrum absorption around 0.361 and the c-Si has overall solar absorption around 0.321 . 


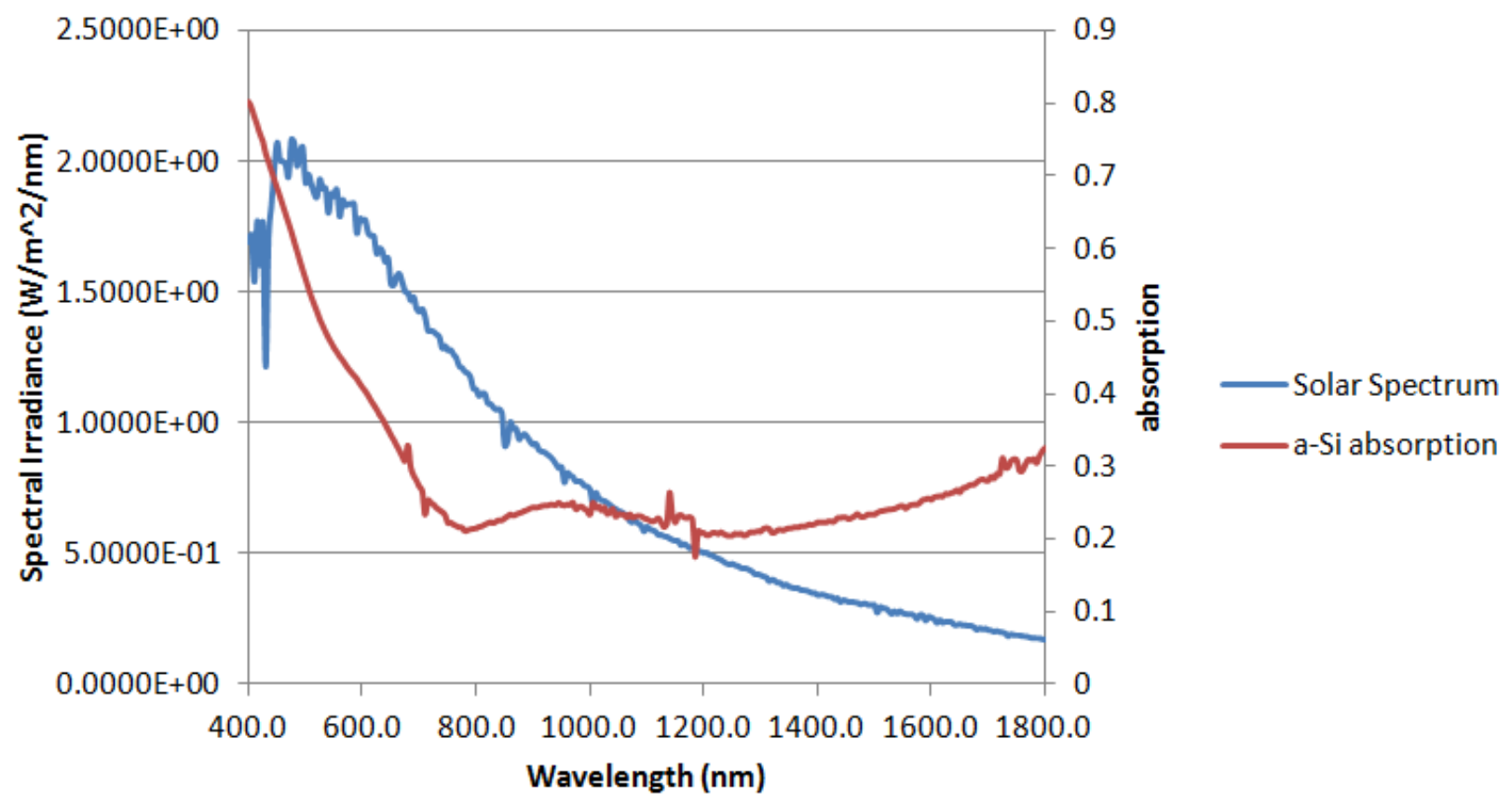

Figure 5.26 a-Si absorption and solar spectral irradiance $v s$ wavelength

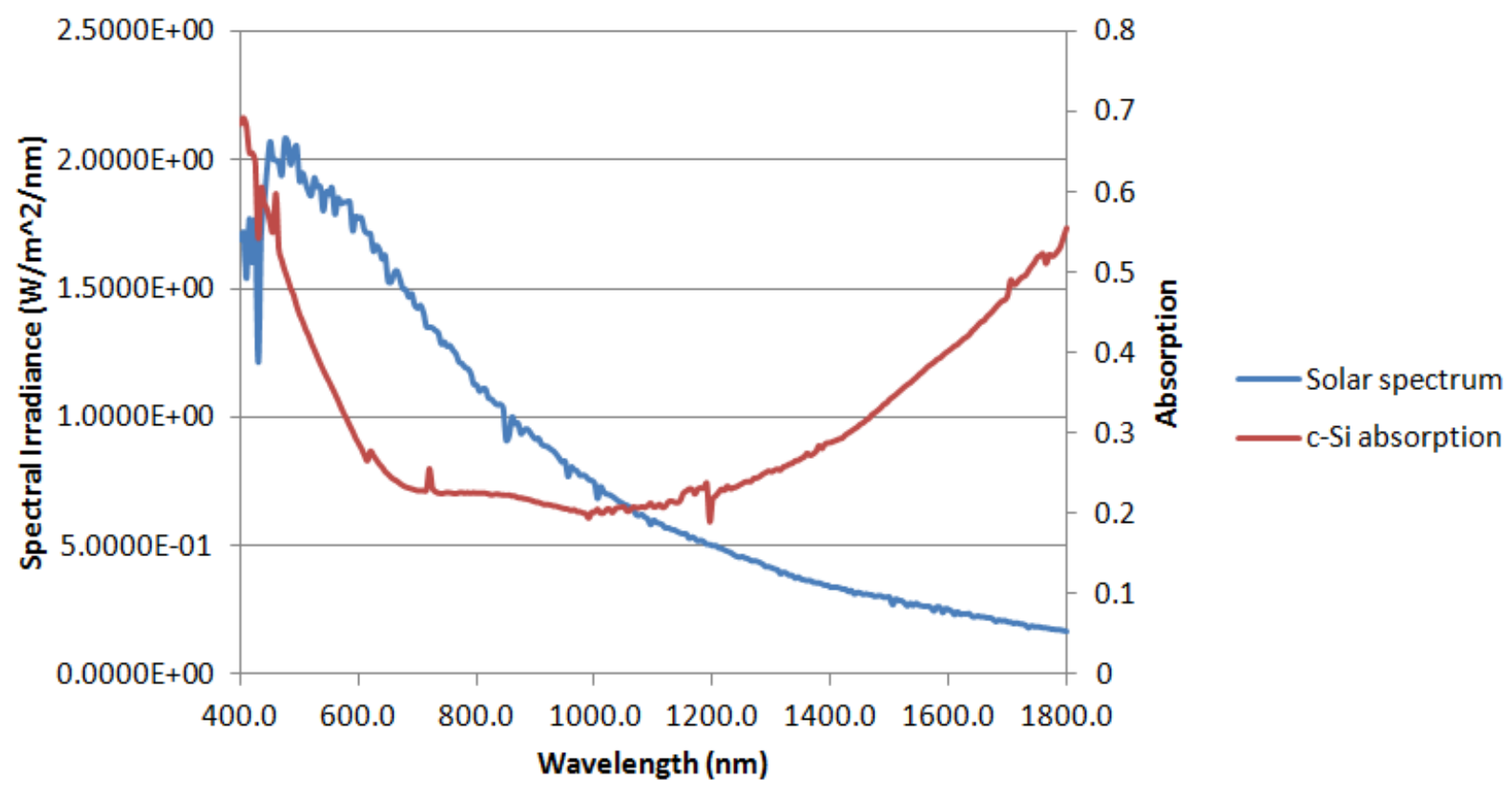

Figure $5.27 \mathrm{c}-\mathrm{Si}$ absorption and solar spectral irradiance $v s$ wavelength 


\section{Chapter VI Conclusion}

In this thesis I have studied an approach to producing a nanostructured Si thin film with the goal of modifying the bandgap to provide a better match to the solar spectrum, and increasing optical absorption. The work involved both sample fabrication and optical characterization of the resulting films. In fabrication, a nanostructured silicon film was prepared by depositing 8nm a-Si on a nanostructured "tree like" ITO substrate produced using VLS-GLAD at University of Alberta. From the SEM image, the aSi film did not conformably coat the ITO "trees", but instead filled in the top branches of the trees, shadowing the structure underneath from further deposition. Although the a-Si film deposition was not as conformal as we would have wished, the fabricated film demonstrated some absorption enhancement in the visible region. This is probably due to the light trapping in the "tree" like ITO structure.

In experimental results, a semiconductor thin film bandgap characterization system was applied successfully. This system measures the optical absorption and reflection of the thin film. Then the absorption coefficient was calculated and used in the estimation of the bandgap of the thin semiconductor film. Comparing the results of the two methods, the estimated bandgaps are quite different. According to the absorption method, the bandgap of the nanostructured $8 \mathrm{~nm}$ a-Si and c-Si film are both around $720 \mathrm{~nm}(1.72 \mathrm{eV})$ while the reflection method indicates that nanostructured a-Si has a bandgap at $825 \mathrm{~nm}(1.5 \mathrm{eV})$ and nanostructured c-Si has a bandgap at $1125 \mathrm{~nm}(1.1 \mathrm{eV})$. The bandgap of the nanostructured amorphous silicon film therefore changed from $1.5 \mathrm{eV}$ to $1.1 \mathrm{eV}$ after the crystallization anneal, coming close to the value expected for 
bulk c-Si. Unfortunately, these two methods do not agree with each other. The reflection method calculated the extinction coefficient and absorption coefficient, which gives more detailed information of light absorbing. The absorption method only provides the percentage of light transmitted and did not consider reflection problems. Also, the free carrier absorption effect follows the absorption method and makes the bandgap unclear at long wavelength. Therefore, the reflection method is more accurate than the absorption method. On the other hand, we can identify the bandgap according to the absorption coefficient and extinction coefficient through the reflection method. Although the absorption method does not provide an accurate solution to determine bandgap, it is helpful to find solar spectrum absorption. According to the result, the nanostructured a-Si film has a absorption around $36.1 \%$ and the nanostructured c-Si film has a absorption around $32.1 \%$.

Overall, the new Si film did not modify the bandgap since the deposition of Si film does conformably coat the nanostructured ITO. The absorption coefficient of the c-Si film is close to the absorption coefficient of the a-Si film, which means the optical absorption of c-Si is successfully increased without Stabler-Wronski degradation. In future work, the deposition of the a-Si film need to be improved to conformably coat the "tree" like ITO.A possible solution is to deposit at higher temperature and lower pressure. 


\section{Reference}

[1] Z. Y. Fan, H. Razavi, J. W. Do, A. Moriwaki, O. Ergen,Y. L. Chueh, P. W. Leu, J. C. Ho, T. Takahashi,L. A. Reichertz, S. Neale, K. Yu, M. Wu, J. W. Ager and A. Javey, Nat. Mater., 2009, $8,648-653$.

[2] Z. Y. Fan, R. Kapadia, P. W. Leu, X. B. Zhang, Y. L. Chueh,K. Takei, K. Yu, A. Jamshidi, A. A. Rathore, D. J. Ruebusch,M. Wu and A. Javey, Nano Lett., 2010, 10, 3823-3827.

[3]Nidhal Mousa Abdul-Ameer,Moafak Cadim Abdulrida. Journal of Modern Physics,2011,2,1530-1537

[4] Garnett, Yang. Light Trapping in Silicon Nanowire Solar Cells. Nano Letters 2010, 10, 10821087

[5] S.M. Sze," Chapter 1 Physics and Properties of Semiconductors," Physics of Semiconductor devices, Second Edition, Newyork, JOHN WILEY \& SONS, 1981

[6] Patterson,James Deane, "Chapter 1 Solid states material," Solid-state physics: introduction to the theory,Second Edition, Berlin ; London : Springer, 2009.

[7] Parker,Michael A, "Introduction to solid State," Solid state and quantum theory for optoelectronics, 1st Edition, Boca Raton, Taylor \& Francis, c2010.

[8]TLP.(2012). Direct and Indirect Band Gap Semiconductors Library[online] . Available: http://www.doitpoms.ac.uk/tlplib/semiconductors/direct.php

[9] San Jose, R.F.Potter, "Semiconductor and Their Nanostructures," Optical characterization techniques for semiconductor technology,2nd edithon, Bellingham, Wash,Society of Photooptical Instrumentation Engineers, 1981.

[10] Mitsuo Fukuda," Basics of Optoelectronic pn-Junction Devices ," Optical Semiconductor Devices, 1st Edition,Wiley-Interscience,December 24, 1998.

[11] Mark Fox ,"Interband absorption," Optical Properties of Solids, 1st edition, Oxford University Press,2001

[12] Mark Fox ," Exciton," Optical Properties of Solids, 1st edition, London,Oxford University Press, 2001

[13] Jacques I.Pankove,"Absorption," optical process in semiconductor, 2nd edition, New Jersey, Prentice-Hall,Inc., 1971.

[14] Randall D.Knight, "Ray Optics,"Physics for Science and Engineers, Custom edition for Carleton University, Pearson Education Inc.,2007

[15]Christiana Honsberg and Stuart Bowden .(2008). Optical property of Silicon [online] . Available: http://pveducation.org/pvcdrom/materials/optical-properties-of-silicon 
[16] Sheffield Hallam University,(2011),Beer's Law[online]. Available:

http://teaching.shu.ac.uk/hwb/chemistry/tutorials/molspec/beers1.htm

[17]Sergey M.Krylov,(2011),Light Absorption-Lambert Beer's Law[online]. Available: http://www.yorku.ca/skrylov/Teaching/BioanalyticalChemistry/Part 3 Using_Light_for_detecti on.pdf

[18] V.Lucarini, J.J.Saarinen K.-E.Peiponen ,E.M. Vartiainen,"Chapter 4 Kramers Kronig Relations and Sum Rules in Linear Optics," Kramers-Kronig Relations in Optical Materials Research, Springer,2004

[19]Haziret DURMUS, Haluk SAFAK, Haldun KARABIYIK," Determination of Optical Constant of Materials by two Different Methods: An Application to Single Crystals Si ," Department of Physics, Faculty of Arts andSciences, Selcuk University Kampus, Konya, 42031, TURKEY

[20] Schubert,(2013),Optical transmittance measurements and bandgap energy [online] Availiable:http://www.ecse.rpi.edu/ schubert/Course-Teaching-modules/A051-Opticaltransmittance-and-bandgap-energy.pdf.

[21] D.M.ROESSLER,"Kramer-Kronig analysis of reflectance data III Approximations, with reference to sodium iodide", Wheatstone Physics Laboraroty, University of London, King's College 22nd February 1966

[22] San Jose, R.F.Potter, "Methods for obtaining the optical constants of a material," Optical characterization techniques for semiconductor technology,2nd edithon, Bellingham, Wash,Society of Photo-optical Instrumentation Engineers, 1981.

[23]G K MThutupalli'r and S G Tomlin," The optical properties of amorphous and crystalline silicon"Department of Physics, University of Adelaide, Australia, 5001, Received 12 July 1976, in final form 9 August 1976

[24]M.A. Green, 'Self-consistent optical parameters of intrinsic silicon at 300K including temperature coefficients', Solar Energy Materials \& Solar Cells 92, pp. 1305-1310, 2008.

[25] J.C. Sturm, Y. Huang, L. Han, T. Liu, B. Hekmatshoar," Amorphous Silicon: The other Silicon "Department of Electrical Engineering Princeton Institute for the Science and Technology of Materials Princeton University, Princeton, NJ 08544, USA

[26]Green MA, Keevers MJ. Optical properties of intrinsic silicon at $300 \mathrm{~K}$. Progress in Photovoltaics: Research and Applications. 1995 ;3:189 - 192.

[27]Z.C. Holman, A. Descoeudres, L. Barraud, F.Z. Fernandez, J.P. Seif, S. De Wolf and C. Ballif, 'Current losses at the front of silicon heterojunction solar cells', IEEE Journal of Photovoltaics 2(1), pp. 7-15, 2012.

[28]Nann, T., Polymers and Adhesives in Microelectronics and Photonics, 2001. First International IEEE Conference on , 2001, 49-53 
[29] Tracie J. Bukowski, Quantum Dot Research: Current State and Future Prospects,2002, Critical Reviews in Solid State and Materials Sciences, 119-142(2002)

[30] Tracie J. Bukowski, T.M. Neidt, Romulo Ochoa and Joseph H. Simmons, Journal of NonCrystalline Solids, 274, 87, 2000

[31]AL Beaudry,RT Tucker.(2012). Indium tin oxide nanowhisker morphology control by vapour-liquid-solid glancing angle deposition, Nanotechnologu 23(2012) 105608

[32] Fraser D B and Cook H D 1972 J. Electrochem. Soc.

[33] Steele J J and Brett M J 2006 J. Mater. Sci., Mater. Electron.18 367-79

[34] Hawkeye M M and Brett M J 2007 J. Vac. Sci. Technol. A25 1317

[35] Wagner R and Ellis W 1964 Appl. Phys. Lett. 4 89-90

[36] Yumoto H, Hatano J, Watanabe T, Fujikawa K and Sato H 1993 Japan. J. Appl. Phys. 32 1204-9

[37] Sherman A 1987 Chemical Vapour Deposition for Microelectronics (Park Ridge, NY: Noyes)

[38] M.Z. Lai, Thermal effects on LPCVD amorphous silicon ,Thin Solid Films 504 (2006) 145 $-148$

[39]Taylor, Antoinette J,Prasankumar and Rohit P," Chapter 4 Methods for Obtaining the OpticalConstants of a Material." Optical techniques for solid-state materials characterization, Boca Raton, Talyor \& Francis Group,LLC,2012

[40]THORLABS ,2008,Illumination Sources [online]

Availiable:http://www.thorlabs.de/newgrouppage9.cfm?objectgroup_id=7541

[41] Richard A.Paselk,(1999). Instrumentation for Optical Spectroscopy, [online] Availiable: http://users.humboldt.edu/rpaselk/C432.S08/Lab/Lab\%20Lectures/C431L_SpecInst.htm

[42]Newport, Grating Physics, [online] Availiable:

http://www.newport.com/Grating-Physics/383720/1033/content.aspx

[43] C. Bolakis, D. Grbovic, N. Lavrik, and G. Karunasiri, "Design and characterization of terahertz-absorbing nano-laminates of dielectric and metal thin films," Opt. Express 18, 1448814495 (2010).

[44] ELECTRICAL AND OPTICAL x8643 PROPERTIES OFHYDROGENATED AMORPHOUSSILICON,J. I. Pankove and D. E. CarlsonRCA Laboratories, Princeton, New Jersey 08540 


\section{Appendix A}

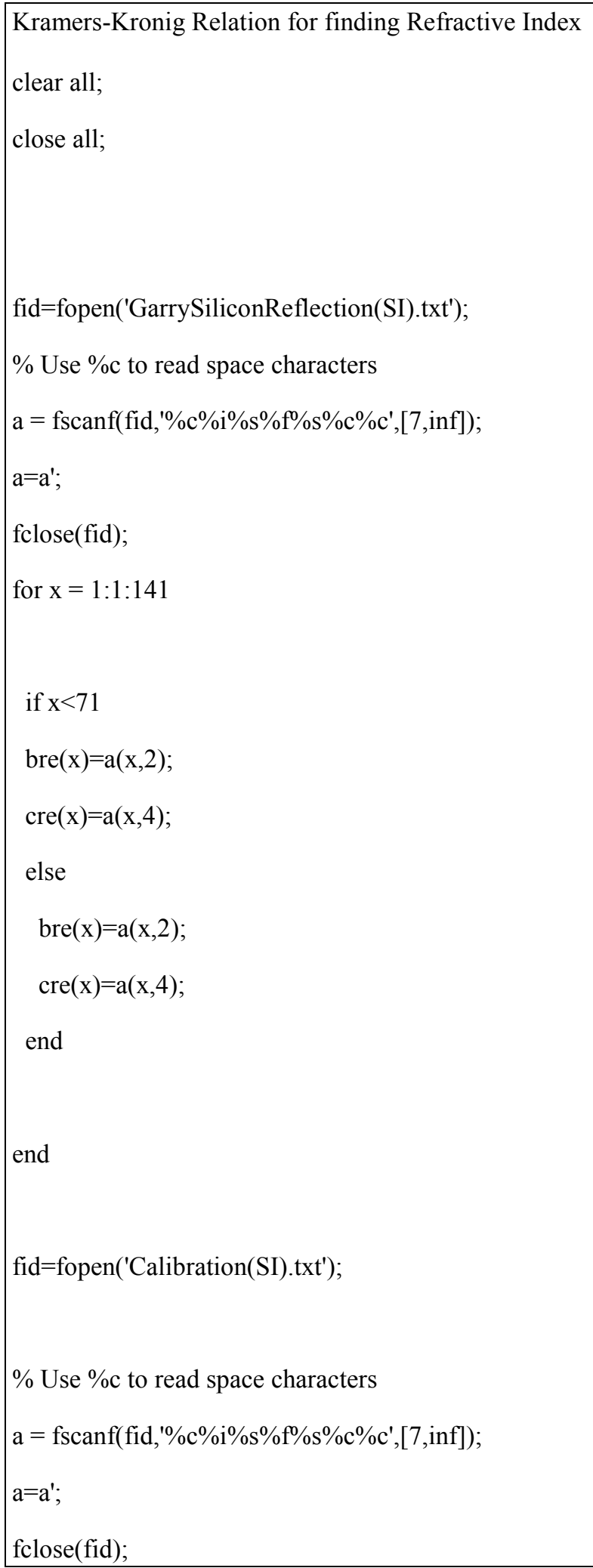




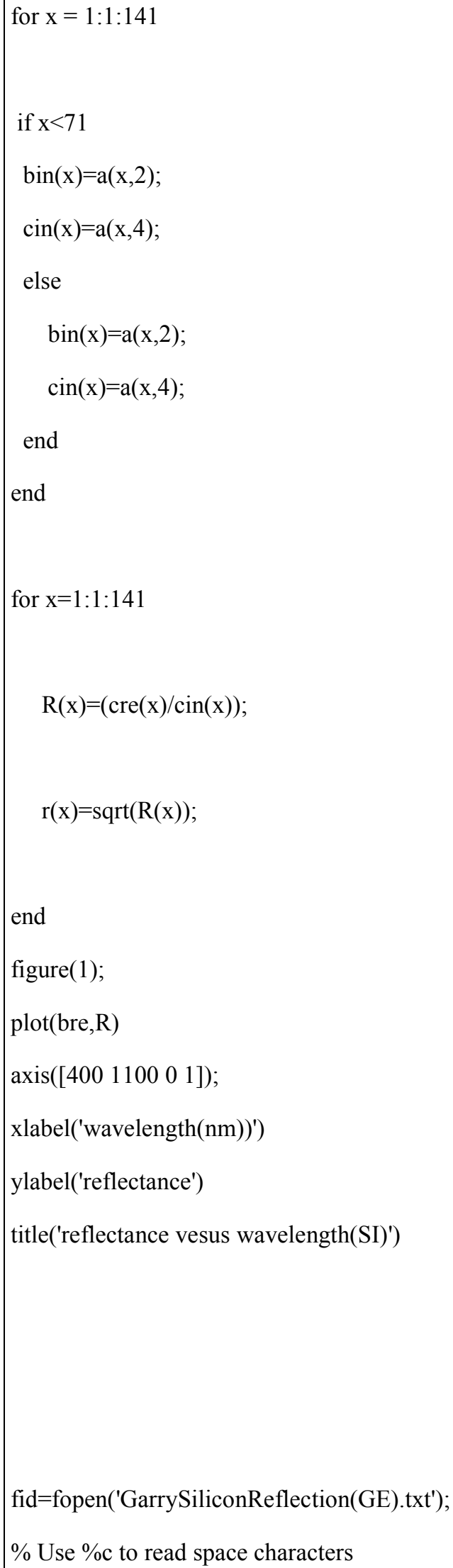




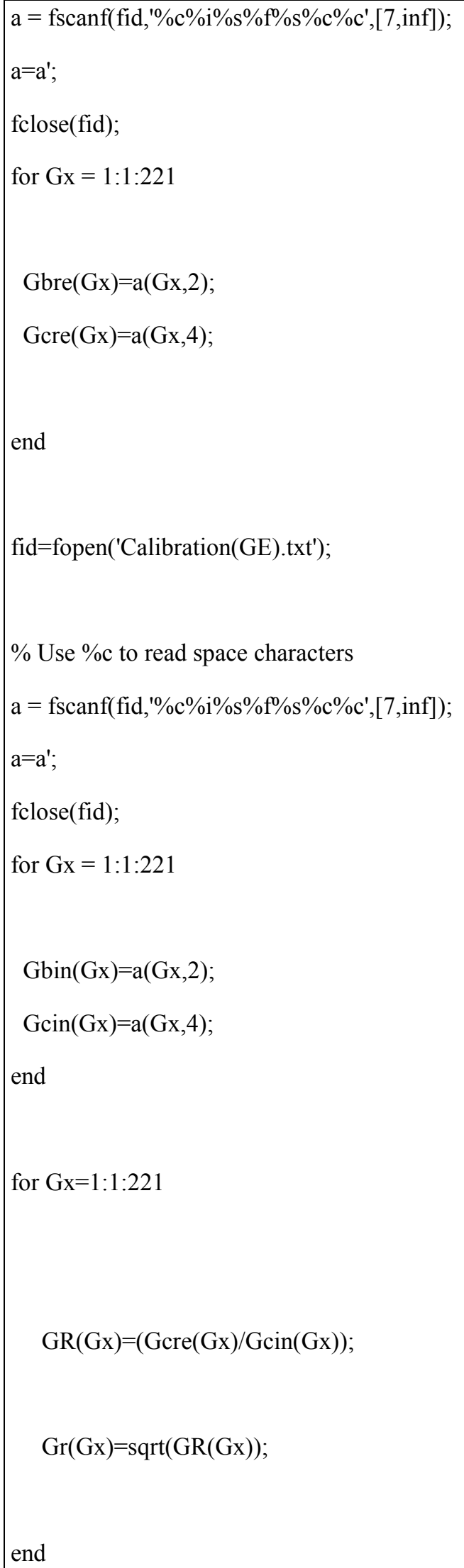




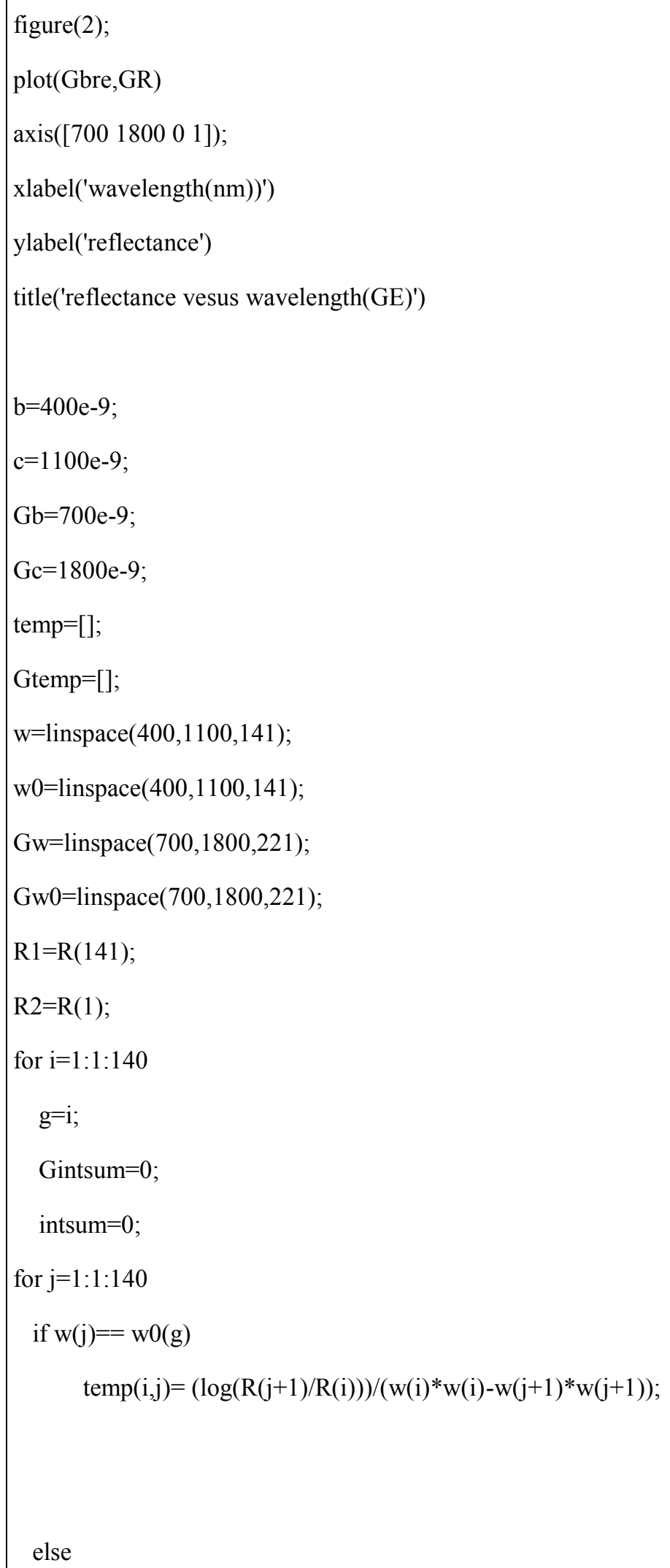




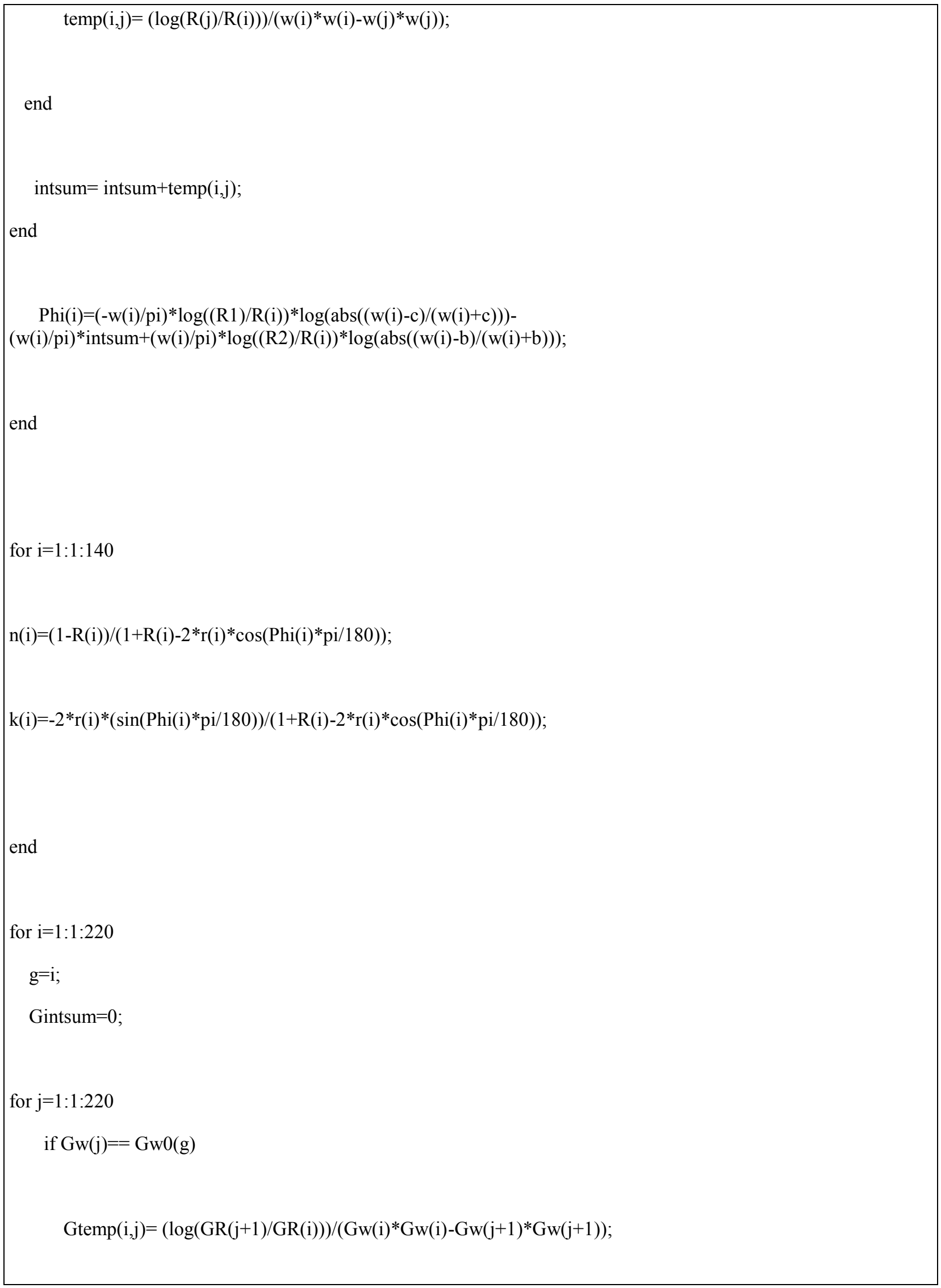




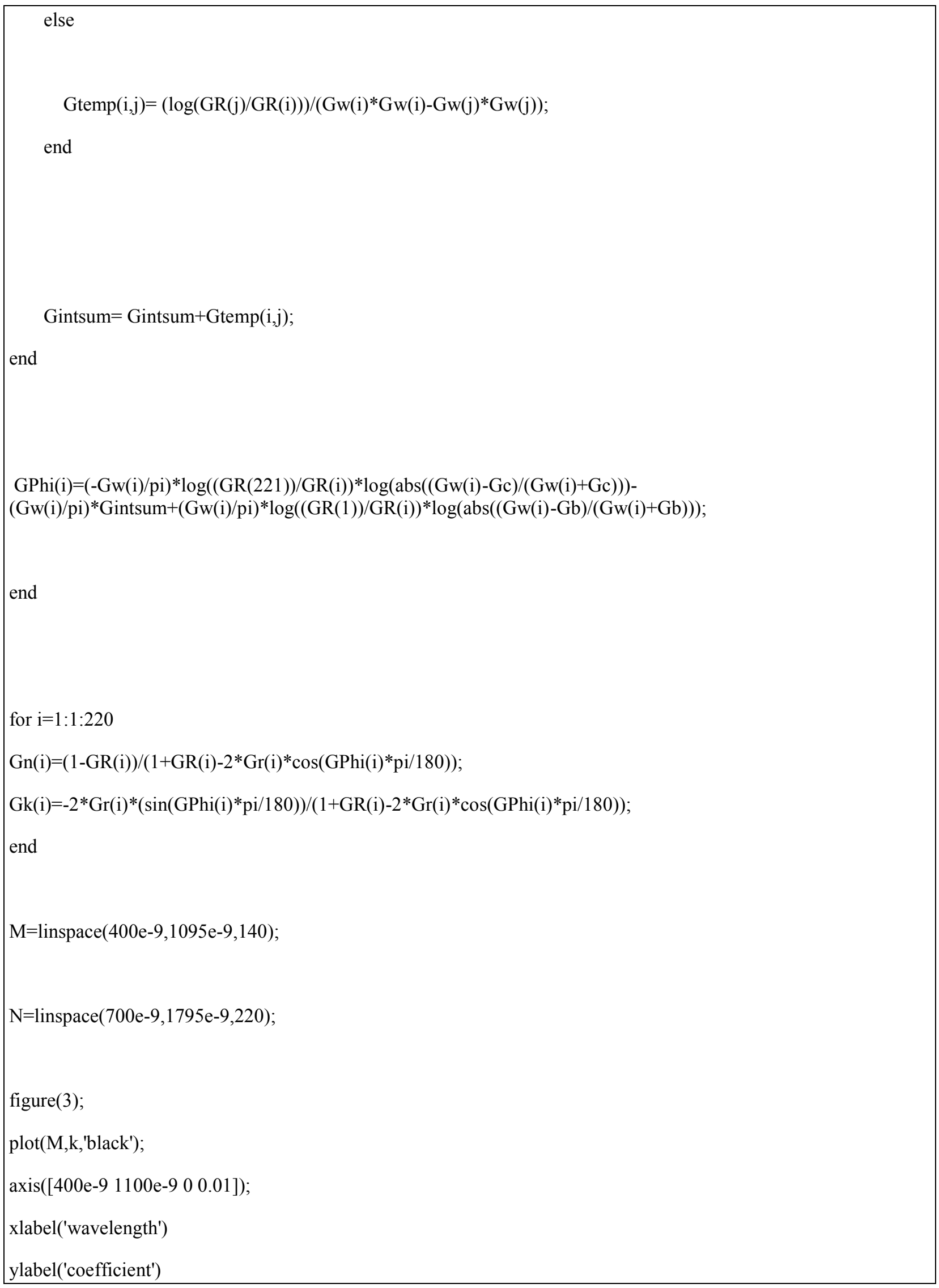




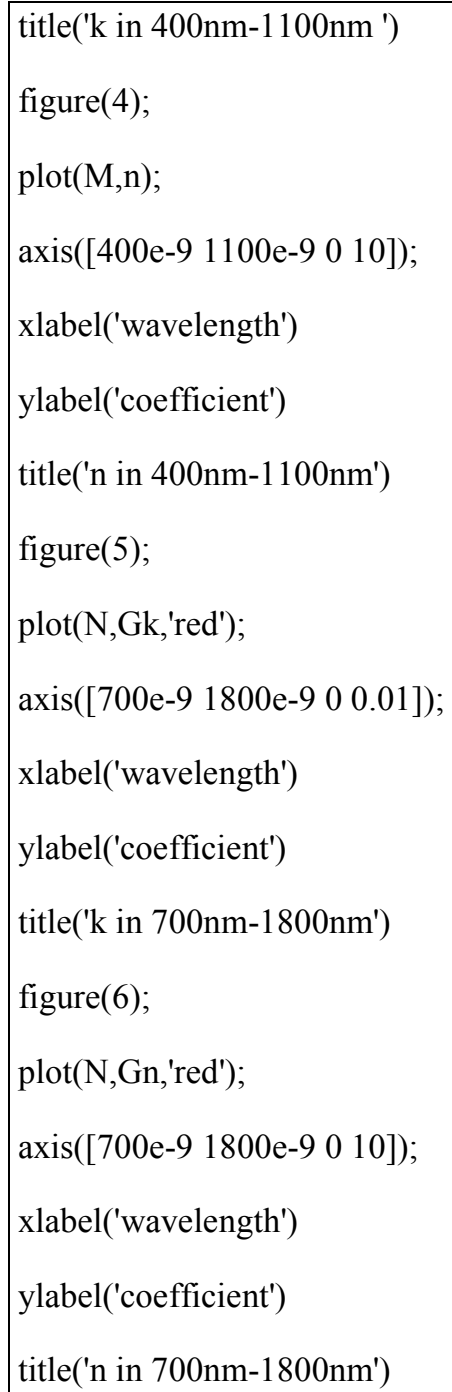

